Article

\title{
Developing a Library of Shear Walls Database and the Neural Network Based Predictive Meta-Model
}

\author{
Mohammad Javad Moradi ${ }^{1}$ (D) and Mohammad Amin Hariri-Ardebili ${ }^{2, *}$ (D) \\ 1 Department of Civil Engineering, Razi University, Kermanshah 67144-14971, Iran; \\ javad_moradi1988@yahoo.com \\ 2 Department of Civil Environmental and Architectural Engineering, University of Colorado, Boulder, \\ CO 80302, USA \\ * Correspondence: mohammad.haririardebili@colorado.edu; Tel.: +1-303-990-2451
}

Received: 26 May 2019; Accepted: 19 June 2019; Published: 23 June 2019

\begin{abstract}
There is a large amount of useful information from past experimental tests, which are usually ignored in test-setup for the new ones. Variation of assumptions, materials, test procedures, and test objectives make it difficult to choose the right model for validation of the numerical models. Results from different experiments are sometimes in conflict with each other, or have minimum correlation. Furthermore, not all these information are easily accessible for researchers and engineers. Therefore, this paper presents the results of a comprehensive study on different experimental models for steel plate and reinforced concrete shear walls. A unique library of up to 13 parameters (mechanical properties and geometric characteristics) affecting the strength, stiffness and drift ratio of the shear walls are gathered including their sensitivity analysis. Next, a predictive meta-model is developed based on artificial neural network. It is capable of forecasting the responses for any desired shear wall with good accuracy. The proposed network can be used to as an alternative to the nonlinear numerical simulations or expensive experimental test.
\end{abstract}

Keywords: steel plate shear wall; reinforced concrete shear wall; meta-model; neural network

\section{Introduction}

Bertero [1] defined the shear wall (SW) as a very stiff member, with high resistance to deformation under load. "It is essentially a plate loaded in its own plane, but the analysis needed to predict its behavior is more complicated than that of simple plates" [1]. Shear walls resist lateral forces parallel to their plane. The slender walls (where the bending deformation is of concern) resist the loads due to cantilever action. In general, the SW system can be classified as reinforced concrete shear walls (RCSW) [2-4], steel plate shear wall (SPSW) [5,6], masonry shear walls [7-9], composite shear walls, and timber plate shear walls $[10,11]$. Although the RCSWs have longer history than SPSWs in structural engineering (due to their remarkable strength and lateral stiffness), the application of SPSWs has also increased in the past few decades $[12,13]$. SPSWs reduce the overall steel consumption, they are lighter compared to other walls, and they can be easily adopted in existing or damaged buildings [14,15]. SPSWs were also added to the design codes as an acceptable lateral load bearing systems [16,17].

The proper design of a SW can provide energy absorption, stiffness, ductility, and appropriate behavior under cyclic/seismic loading. The adequate stiffness of the lateral load bearing system may reduce the story drift and fulfill the code requirements. Quantification of the strength, stiffness and ductility leads to a precise performance evaluation of the SW systems.

Design and/or analysis of a SW system requires proper background information about the mechanism and performance of these systems. Often, the numerical models should be validated based on the experimental tests [18]. There is a very large set of SW experiments tested by different 
researchers with various assumptions and purposes. The results are sometimes in conflict with each other or at least have minimum correlation. This makes the model selection quite difficult. In addition, not all these information are easy to collect and process by engineers (as they might be copyrighted or restricted materials). The authors have been confronted by such a problems in their previous studies on both steel and RC shear walls from experimental [15] and numerical [19] points of view. This was one of the motivations to develop such a comprehensive database of SW models, which would be useful for all the future studies related to the experimental and numerical validations.

On the other hand, such a huge database might be difficult to track by practitioners, and, thus, a meta-model is required to summarize and present the results in a systematic way. This should include the parameters affecting the stiffness and strength of the SWs, as well as their sensitivity. There exist many soft computing (or surrogate) techniques with different levels of sophistication that can be used to post-process a database [20]: artificial neural network (ANN), support vector machine [21], polynomial chaos expansion [22], etc. In this research, the ANN is selected, which has been proven to be one of the best soft computing techniques, in nearly all engineering fields. The neural network can predict several outputs by receiving a set of input parameters. To do this, a neural network should be trained efficiently. Then, it can predict other results that have not been investigated before.

\section{Research Significance and Contributions}

The research significance can relay on the profuse investigation of the existing database of the SWs, as well as to provide a useful tool to fellow researchers. It is important to notice that the difficulty in obtaining large scale results for SWs behavior has greatly limited the research in the past for this important issue. This paper is intended to adopt one of the widely-used and well-established soft computing techniques (i.e., ANN) to solve an engineering problem. Comparing and contrasting various surrogate models are not the focus of this paper. The developed predictive meta-model can be used as a useful alternative in the absence of detailed experiments or numerical simulations. The contribution of the authors in this paper can be summarized as follows:

- Developing a large library of SW experimental tests for both the steel plate and reinforced concrete materials

- Training and developing an ANN-based meta-model for SWs for response prediction purposes

- Providing an active library of data which can be easily updated by any new information

- Performing sensitivity analysis of the responses to the input parameters

- Proposing a predictive models for stiffness and strength for both types of SWs, as well as the drift ratio for RCSWs

The paper starts with a brief description on ANN model and its fundamental features in Section 3 (for those readers who are not familiar with this concept). Next, the comprehensive database is introduced in Section 4. The ANN training process and meta-models are then presented in Section 5 followed by the sensitivity analysis. Finally, Section 6 provides the general conclusions and recommendations. Detailed information about the SW library, and the developed neural network model can be found in Appendixes A and B, respectively.

\section{Artificial Neural Network}

An ANN is inspired by the human brain, in which the neurons are tasked with data processing. By gathering these neurons, the layer is produced, and an ANN may consist of several layers. Neurons are connected by weighted synapse. Finally, the input data exit from the output layer after applying numerical processing on them [23,24]. Hebb [25], Widrow and Hoff [26] and Rosenblatt [27] offered fundamentals and extensions of the ANN. Research on the neural network continued until 1985, and Rumelhart et al. [28] proposed multi-layer perceptron (MLP) with back-propagation algorithm. Subsequently, various models and networks were presented and research is still ongoing [29]. 
A MLP is a type of neural network in which neurons are deployed on more than one layer. An output of $n$th neuron in the $m$ th layer in a generic MLP is determined as:

$$
O_{n}^{m}=f_{n}^{m}\left(\left(w^{(m-1)}\right)^{T} O_{n}^{m-1}+b^{m}\right)
$$

where $f_{n}^{m}$ is the function of $n$th neuron in the $m$ th layer, $w^{(m-1)}$ is the weights from $(m-1)$ th to $m$ th layer, and $b^{m}$ is $m$ th layer's bias term.

For the last layer, $n=1$ and $O_{n}^{m}=\hat{y}$. The objective function for learning process is defined in a way to minimize the mean squared error (MSE $\left.=\frac{1}{2 N} \sum(y-\hat{y})^{2}\right)$ between the observed value, $y$, and the estimated one, $\hat{y}$. This is an iterative procedure by initializing $w$ value, estimating $\hat{y}$, and computing the corresponding MSE. If the obtained MSE falls outside the satisfactory range, the initial $w$ should be updated.

The MLP is capable of classification, clustering, and function approximation. With a neural network of three [30-33] and four [34] layers, and sufficient number of neurons in the hidden layers, nearly any function can be estimated $[23,35]$. Usually, a four-layer network requires fewer weights to estimate the functions; however, it introduces more local minima [36].

The ANN is one of popular soft computing techniques in structural engineering and mechanics. Although there is much research and development, on both the theoretical aspects and application, some of the most relevant ones are reviewed in this section. Adeli and Seon Park [37] evaluated the maximum moment of a beam. Xu et al. [38] proposed a method for crack detection in plates using a MLP. De Lima et al. [39] evaluated three types of steel beam-column joints. It was observed that the neural network can properly estimate the bending strength of the connection; however, due to differences in the measurement systems, the network had extra error in estimation of the stiffness parameter. Abdalla et al. [40] provided an estimate of the shear strength of RC rectangular beams by examining the parameters such as concrete strength, reinforcement ratio, thickness, length, and width of the beam. It was observed that the network can estimate the shear strength of concrete beam with acceptable accuracy. More than $96 \%$ of the estimated neural network data had absolute error less than $15 \%$, while it was $26 \%$ and $23 \%$ for UK and US standards estimated data, respectively. Kumar et al. [41] evaluated composite shell vibration. It was found that the response from the neural network for all of the loading patterns in the research was nearly identical to the response.

González and Zapico [42] determined the seismic failure in structures. The network inputs were the natural frequency of the structure and shape of the mode, and the output was mass and stiffness. The data were obtained using finite element analysis. The network showed a good performance in prediction of the structural behavior. Yan et al. [43] reviewed the structural failure of the beams using back propagation algorithm. It was observed that the neural network properly predicts the failure of the beams. Lee and Lee [44] predicted the shear strength of the RC beam reinforced with FRP by evaluating 106 experimental data using ANN. It was observed that the network can estimate shear strength with better accuracy than other available equations. Asteris and Plevris [45] proposed the application of ANNs to approximate the failure surface of the anisotropic masonry materials in a dimensionless form. They compared the derived results with experimental findings and analytical solutions, and reported a reliable performance for the ANN-based approximation of the masonry failure surface under biaxial stress. Toghroli et al. [46] reviewed various numerical methods to examine the parameters affecting the bearing capacity of composite beams. It was observed that extreme learning machine has the best result in estimating the composite beam behavior among other investigated methods. Naderpour et al. [47] proposed a method in which the geometric and mechanical properties of cross-section and FRP bars, and shear span-depth ratio were considered for concrete beams. The error in the shear strength estimation was about $9.7 \%$, which was significantly lower than other methods and relationships.

Rezaei Rad and Banazadeh [48] presented a comprehensive application of probabilistic soft computing technique in damage determination of steel structure. Ghorbani et al. [49] used the MLP and radial basis function ANNs to predict the support pressure, and develop the ground motion curve in an underground structure (circular tunnel). An elastoplastic, strain-softening rock mass 
was considered including both single- and double-layer hidden neurons. Asteris and Kolovos [50] proposed the application of ANNs to predict the mechanical characteristics of self-compacting concrete. The 28-day compressive strength of admixture-based self-compacting concrete was predicted, and a formula was proposed for the data normalization. Chen et al. [51] developed two hybrid surrogate models by combining ANN with imperialist competitive algorithm (ICA) and genetic algorithm. These techniques were used to predict the safety factor of retaining walls in dynamic condition. A very large database of 8000 designs were used for this purpose. They found that the ICA-ANN provides a better performance. Finally, Asteris and Nikoo [52] optimized the connection weights of the feed-forward ANNs using the artificial bee colony (ABC). The algorithm was then used to determine the fundamental period of reinforced concrete infilled structures. They confirmed the superior performance of this hybrid method over the traditional ones.

\section{Library of Shear Wall Database}

As stated in Section 1, a comprehensive library of SW databases is collected. It includes about 300 samples with 12 features for SPSWs, and about 4000 samples with 13 features for the RCSWs. Those features are listed in Tables 1 and 2. It should be noted that the SPSW models are without stiffener, slip, and opening and are non-corrugated.

Table 1. Statistics of the parameters in SPSW model.

\begin{tabular}{lcccccc}
\hline Parameter & Symbol & Unit & Min & Max & Mean & STD \\
\hline Frame Height & $h_{f}$ & $\mathrm{~mm}$ & 500 & 1830 & 1073 & 283 \\
Frame Width & $b_{f}$ & $\mathrm{~mm}$ & 500 & 3660 & 1747.8 & 712.2 \\
Column Area & $A_{c}$ & $\mathrm{~mm}^{2}$ & 1070 & 18190 & 7598.5 & 4424.9 \\
Column Moment of Inertia & $I_{c}$ & $\mathrm{~mm}^{4}$ & 864,000 & $3.47 \times 10^{8}$ & $7.00 \times 10^{7}$ & $8.70 \times 10^{7}$ \\
Beam Area & $A_{b}$ & $\mathrm{~mm}^{2}$ & 1070 & 16,300 & 6163.1 & 3682 \\
Beam Moment of Inertia & $I_{b}$ & $\mathrm{~mm}^{4}$ & 864,000 & $6.37 \times 10^{8}$ & $6.10 \times 10^{7}$ & $1.20 \times 10^{8}$ \\
Plate Height & $h_{p}$ & $\mathrm{~mm}$ & 380 & 1500 & 1034 & 318.3 \\
Plate Width & $b_{p}$ & $\mathrm{~mm}$ & 380 & 3337 & 1360.8 & 668.8 \\
Plate Thickness & $t_{p}$ & $\mathrm{~mm}$ & 0.5 & 15 & 3.79 & 3.12 \\
Plate Yield Stress & $F_{y p}$ & $\mathrm{~Pa}$ & 295,000 & $4.20 \times 10^{8}$ & $2.50 \times 10^{8}$ & $1.00 \times 10^{8}$ \\
Plate Ultimate Stress & $F_{u p}$ & $\mathrm{~Pa}$ & $2.60 \times 10^{8}$ & $5.60 \times 10^{8}$ & $4.10 \times 10^{8}$ & $9.60 \times 10^{7}$ \\
Plate Modulus of Elasticity & $E_{p}$ & $\mathrm{~Pa}$ & $1.50 \times 10^{11}$ & $2.00 \times 10^{11}$ & $1.80 \times 10^{11}$ & $2.20 \times 10^{10}$ \\
Load Bearing Capacity & $P$ & $\mathrm{~N}$ & 11,480 & $3,020,000$ & $1,056,303$ & 795,832 \\
Lateral Stiffness & $K$ & $\mathrm{~N} / \mathrm{mm}$ & 2380 & 181,000 & $68,983.2$ & 45,897 \\
\hline
\end{tabular}

Table 2. Statistics of the parameters in RCSW model.

\begin{tabular}{lcccccc}
\hline Parameter & Symbol & Unit & Min & Max & Mean & STD \\
\hline Axial Load & $A . L$ & $\mathrm{kN}$ & 0 & 3192 & 323 & 514.1 \\
Boundary Element Length & $W_{1 b}$ & $\mathrm{~mm}$ & 0 & 380 & 123.6 & 99.6 \\
Boundary Element Width & $W_{2 b}$ & $\mathrm{~mm}$ & 0 & 610 & 142.7 & 129 \\
Vertical Column Reinforcement Ratio & $\rho_{v c}$ & $\%$ & 0 & 14.33 & 2.3 & 2.62 \\
Horizontal Column Reinforcement Ratio & $\rho_{c h}$ & $\%$ & 0 & 6.69 & 0.75 & 0.93 \\
Yield Stress of Column Reinforcement & $F_{y c}$ & $\mathrm{MPa}$ & 276 & 1412 & 495.6 & 181.6 \\
Wall Height & $h_{w}$ & $\mathrm{~mm}$ & 476 & 11,760 & 1812.5 & 1210.3 \\
Wall Width & $l_{w}$ & $\mathrm{~mm}$ & 450 & 5400 & 1389.2 & 685.2 \\
Wall Thickness & $t_{w}$ & $\mathrm{~mm}$ & 45 & 240 & 115 & 47 \\
Vertical Wall Reinforcement Ratio & $\rho_{v w}$ & $\%$ & 0 & 14.33 & 0.74 & 1.47 \\
Horizontal Wall Reinforcement Ratio & $\rho_{h w}$ & $\%$ & 0 & 6.69 & 0.47 & 0.54 \\
Yield Stress of Wall Reinforcement & $F_{y w}$ & $\mathrm{MPa}$ & 216 & 1412 & 503.2 & 190.2 \\
Concrete Compressive Strength & $f_{c}^{\prime}$ & $\mathrm{MPa}$ & 9.5 & 93.6 & 35.3 & 14.6 \\
Lateral Load Bearing & $P$ & $\mathrm{kN}$ & 15.42 & 3230.7 & 530.2 & 500 \\
Lateral Stiffness & $K$ & $\mathrm{kN} / \mathrm{mm}$ & 3.29 & 2933.7 & 209 & 341.8 \\
Drift Ratio & $D$ & $\%$ & 0.21 & 6.9 & 1.65 & 1.05 \\
\hline
\end{tabular}


Figure 1 illustrates the dimensions used for a generic SPSW and RCSW. Tables A1 and A2 (see Appendix A) illustrate the detailed library of SW database for steel plate and reinforced concrete walls, respectively. Feature selection should be included in all the aspects of the problem. The input parameters are in a wide range of variations, and should be normalized before using in the meta-model. Therefore, the data were linearly normalized in range of $[0,1]$. This linear transformation preserves all the relationships of the initial database [53]. Initial weights were selected randomly focusing on the range of $[-0.77,+0.77]$. It has been empirically observed that this weight initialization technique leads to better performance and faster training of the ANN [54].

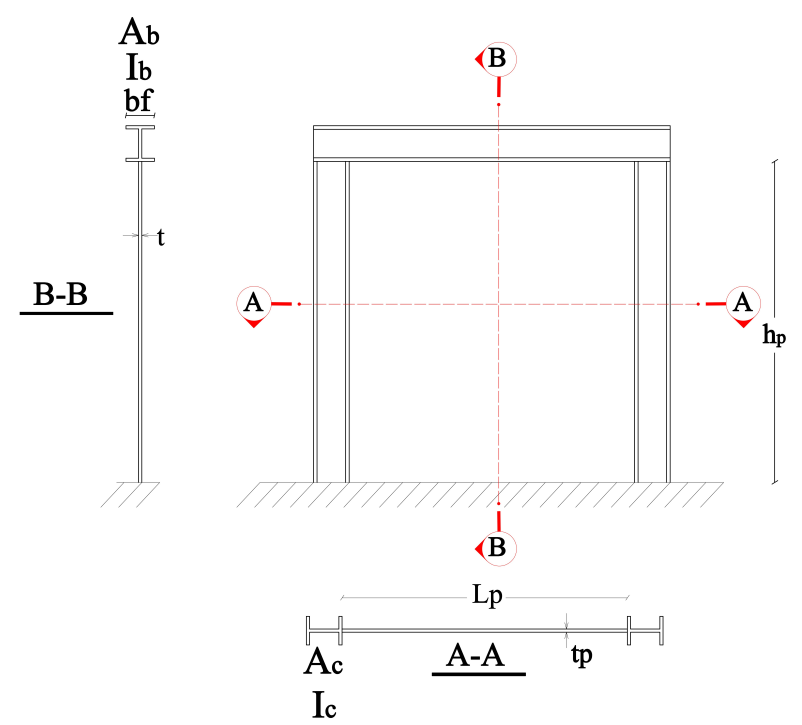

(a)

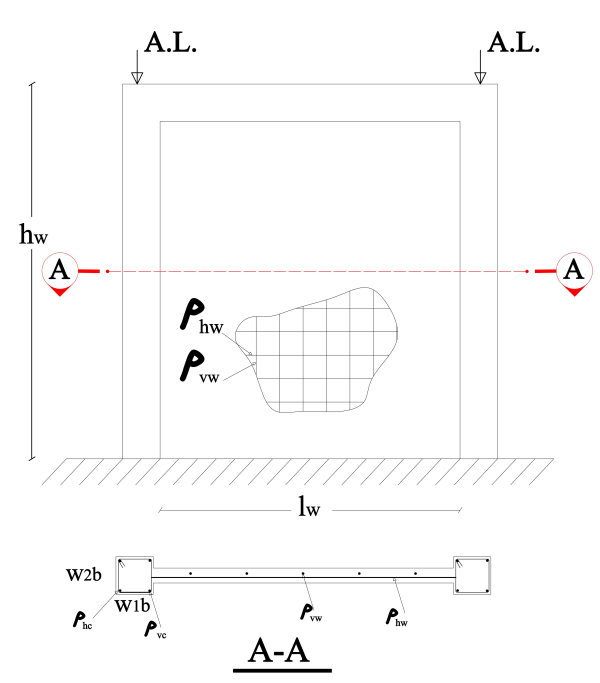

(b)

Figure 1. Dimensions of a generic shear wall. (a) SPSW, (b) RCSW.

A poor weight initialization may cause divergence of the outputs or becoming stuck at the local minima [23]. Tables 1 and 2 show the statistics of the selected features for SPSWs and RCSWs, respectively. The target data were considered to be the maximum strength, $P$, and stiffness, $K$, for SPSWs, plus drift ratio, $D$, (in addition to $K$ and $P$ ) for RCSWs. The choice of these parameters was based on the abundance of data (e.g., crack pattern) in the literature, as well as the proper SW behavior expression. By estimating these parameters, the geometric characteristics and the mechanical properties required to obtain the desired shear strength, stiffness and drift ratio can be determined (accounting for an appropriate reliability coefficient). In design of SPSWs, it is assumed that the boundary elements do not yield, thus the mechanical properties of these members are not included as a network input. In all experiments, the buckling and the collapse of the infill plate occurred prior to the boundary elements. The network is applicable to the initial design of the SWs, as well as to retrofit the existing ones.

\section{Modeling the Network}

\subsection{Number of Neurons}

When the desired performance of the network is accomplished, the learning process ends. On the other hand, the number of optimal neurons required for each layer is not known beforehand, and it is usually determined by trial and error. The constructive approach is used to overcome this problem. This method finds the smallest network that can provide the required performance, and is suitable for passing local responses (or local minima); however, it is time consuming [55]. Empirical research has been performed to determine the optimal number of neurons [56]. A summary of this research is presented in Table 3. The results of this table can be used to determine the range of changes in the 
number of the optimal neurons, and also is a limit for the interval of trial and error. As can be seen, the number of neuron in the hidden layer range from 2 to 39. The network's MSE value is computed for each number of neurons in the hidden layer, while other parameters are kept constant.

Table 3. A survey on number of the hidden neurons.

\begin{tabular}{llcc}
\hline Reference & Criteria for Neuron of Hidden Layer & SPSW & RCSW \\
\hline Hecht-Nielsen [57] & $\leq 2 N_{i}$ & $\leq 24$ & $\leq 26$ \\
Hush [58] & $3 N_{i}$ & 36 & 39 \\
Ripley [59] & $\frac{N_{i}+N_{o}}{2}$ & 6.5 & 7 \\
Gallant and Gallant [60] & $2 N_{i}$ & 24 & 26 \\
Wang [61] & $\frac{2 N_{i}}{3}$ & 8 & 8.6 \\
Masters [62] & $\sqrt{N_{i}+N_{o}}$ & 3.6 & 3.7 \\
Paola [63] & $\frac{N_{o} N_{i}+0.5 N_{o} \times\left(N_{o}^{2}+N_{i}\right)-1}{N_{o}+N_{i}}$ & 1.3 & 1.35 \\
Li et al. [64] & $\left(\frac{\sqrt{8 N_{i}}}{2}\right)-2$ & 2.9 & 3.1 \\
Tamura and Tateishi [65] & $N_{i}+1$ & 13 & 14 \\
Lai and Serra [66] & $N_{i}$ & 12 & 13 \\
Nagendra [67] & $N_{i}+N_{o}$ & 13 & 14 \\
Gencay [68] & $\ln \left(N_{i}\right)$ & 2.4 & 2.5 \\
Chris Wong et al. [69] & $\frac{N_{i}+N_{o}}{2}$ & 6.5 & 7 \\
Heaton [70] & $\frac{2 N_{i}}{3}+N_{o}$ & 9 & 9.6 \\
Zhang et al. [71] & $\frac{2^{N_{i}}}{N}+1$ & 14.6 & 3 \\
Huang [72] & $\sqrt{\left(N_{o}+2\right) N_{i}}+2 \sqrt{\frac{N_{i}}{\left(N_{o}+2\right)}}$ & 10 & 10.4 \\
Ke and Liu [73] & $N_{i}+\sqrt{N}$ & 29 & 76 \\
Trenn [74] & $\frac{\left(N_{i}+N_{o}+1\right)}{2}$ & 6 & 6.5 \\
Shibata and Ikeda [75] & $\sqrt{N_{o} N_{i}}$ & 3.4 & 3.6 \\
Hunter et al. [76] & $N_{i}+1$ & 13 & 14 \\
Sheela and Deepa [77] & $\frac{4 N_{i}^{2}+3}{N_{i}^{2}-8}$ & 4.2 & 4.2 \\
\hline & & & \\
\hline
\end{tabular}

Figure 2 shows the performance of the network based on MSE for train and test data as a function of number of neurons. Each ANN model (with particular number of neurons) was iterated 10 times and its mean was used to increase the accuracy of the model. The mean errors for each neuron is also summarized in Table 4 . The best performance among the train and test data for SPSW belonged to the network with 6 neurons, i.e., ANN 12-6-1, where the first, second and third digits are the number of input nodes, hidden neurons, and output nodes, respectively. On the other hand, ANN 13-10-1 had the best performance for RCSW. 


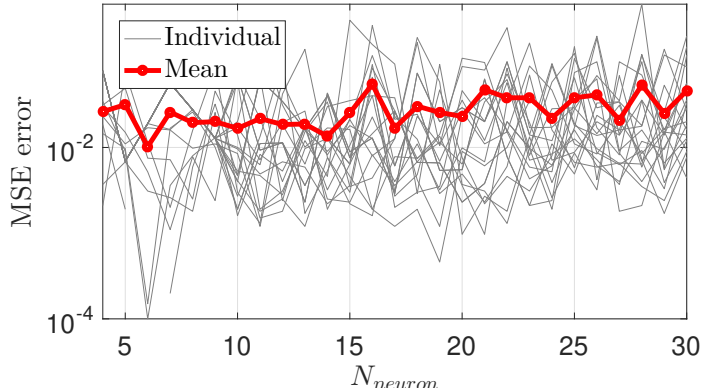

(a)

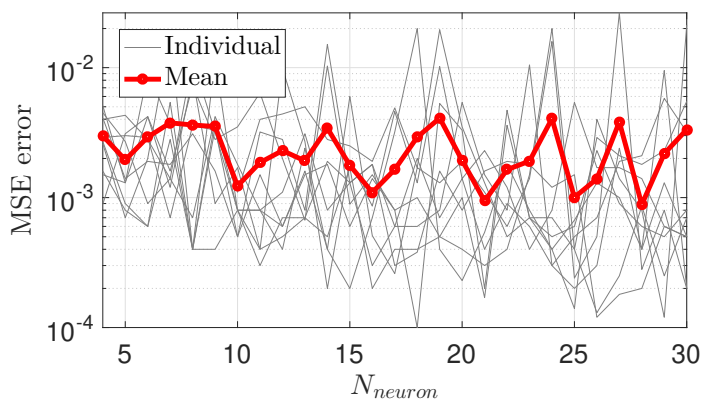

(c)

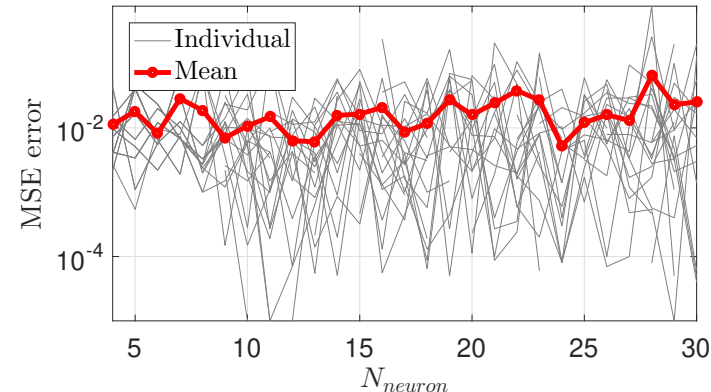

(b)

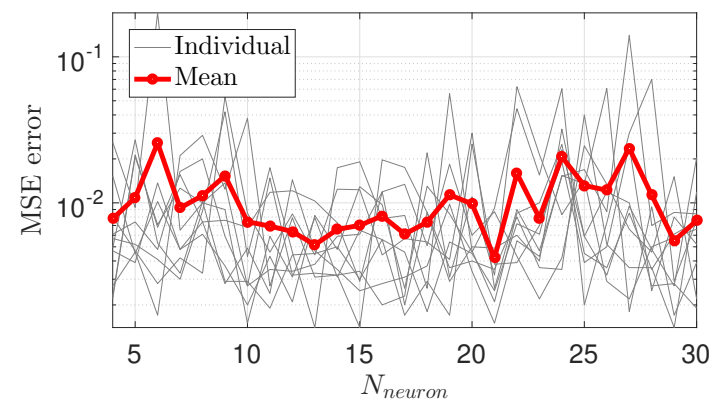

(d)

Figure 2. The network's neuron-dependent MSE. (a) SPSW; train data, (b) SPSW; test data, (c) RCSW; train data, (d) RCSW; test data.

Table 4. Mean MSE for train and test data as a function of number of neurons.

\begin{tabular}{|c|c|c|c|c|}
\hline \multirow{2}{*}{ Neurons } & \multicolumn{2}{|c|}{ SPWS } & \multicolumn{2}{|c|}{ RCSW } \\
\hline & $\begin{array}{l}\text { Train } \\
\text { Data }\end{array}$ & $\begin{array}{l}\text { Test } \\
\text { Data }\end{array}$ & $\begin{array}{l}\text { Train } \\
\text { Data }\end{array}$ & $\begin{array}{l}\text { Test } \\
\text { Data }\end{array}$ \\
\hline 4 & 0.0262 & 0.0113 & 0.0029 & 0.0078 \\
\hline 5 & 0.0316 & 0.0180 & 0.0019 & 0.0109 \\
\hline 6 & 0.0102 & 0.0082 & 0.0029 & 0.0257 \\
\hline 7 & 0.0259 & 0.0285 & 0.0038 & 0.0092 \\
\hline 8 & 0.0195 & 0.0187 & 0.0036 & 0.0112 \\
\hline 9 & 0.0200 & 0.0070 & 0.0035 & 0.0153 \\
\hline 10 & 0.0167 & 0.0107 & 0.0012 & 0.0074 \\
\hline 11 & 0.0217 & 0.0149 & 0.0023 & 0.0069 \\
\hline 12 & 0.0185 & 0.0063 & 0.0019 & 0.0064 \\
\hline 13 & 0.0188 & 0.0061 & 0.0034 & 0.0052 \\
\hline 14 & 0.0135 & 0.0156 & 0.0018 & 0.0066 \\
\hline 15 & 0.0253 & 0.0164 & 0.0011 & 0.0070 \\
\hline 16 & 0.0545 & 0.0208 & 0.0016 & 0.0081 \\
\hline 17 & 0.0169 & 0.0087 & 0.0029 & 0.0061 \\
\hline 18 & 0.0303 & 0.0117 & 0.0041 & 0.0074 \\
\hline 19 & 0.0256 & 0.0273 & 0.0019 & 0.0113 \\
\hline 20 & 0.0230 & 0.0159 & 0.0009 & 0.0099 \\
\hline 21 & 0.0473 & 0.0242 & 0.0016 & 0.0042 \\
\hline 22 & 0.0380 & 0.0380 & 0.0019 & 0.0159 \\
\hline 23 & 0.0380 & 0.0270 & 0.0041 & 0.0079 \\
\hline 24 & 0.0220 & 0.0053 & 0.0010 & 0.0206 \\
\hline 25 & 0.0380 & 0.0120 & 0.0014 & 0.0132 \\
\hline 26 & 0.0410 & 0.0160 & 0.0038 & 0.0122 \\
\hline 27 & 0.0210 & 0.0130 & 0.0009 & 0.0237 \\
\hline 28 & 0.0535 & 0.0648 & 0.0022 & 0.0113 \\
\hline 29 & 0.0251 & 0.0229 & 0.0033 & 0.0055 \\
\hline 30 & 0.0459 & 0.0254 & 0.0019 & 0.0076 \\
\hline
\end{tabular}




\subsection{Performance of the Network}

Once the network is trained, it can be used to define the complex relationship among the input parameters, and to predict the output for any new SW model. Generalization means estimating the value on the hyper-surface where there are no available data. Mathematically, the learning process is a nonlinear curve-fitting algorithm, while generalization is the interpolation and extrapolation of the input data [47]. The ability of a network to generate new outputs depends on the number of parameters involved in the problem, the complexity of the parameters, and the structure of the network.

The neural network is constructed based on the number of neurons obtained from previous section. Figure 3 shows the architecture of the proposed networks for both SWs. In this paper, two MLPs are used to estimate the load bearing capacity, stiffness, and drift ratio of the SPSWs and RCSWs.

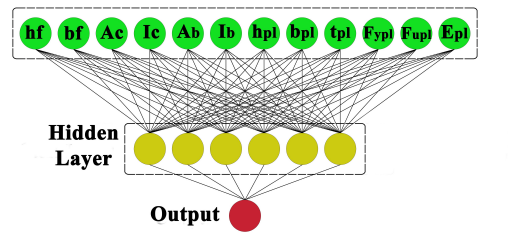

(a)

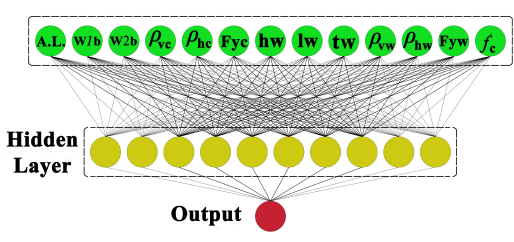

(b)

Figure 3. Proposed ANN models for shear walls. (a) SPSW, (b) RCSW.

Overall, $70 \%$ of data were used for training, $15 \%$ for validation, and $15 \%$ for testing. The validation and test data monitor the network over-training and performance, respectively. Performance of the network is shown in Figure 4. It states that the network has specific MSE for $n$th epoch, and there is no un-convergence or over-fitting.

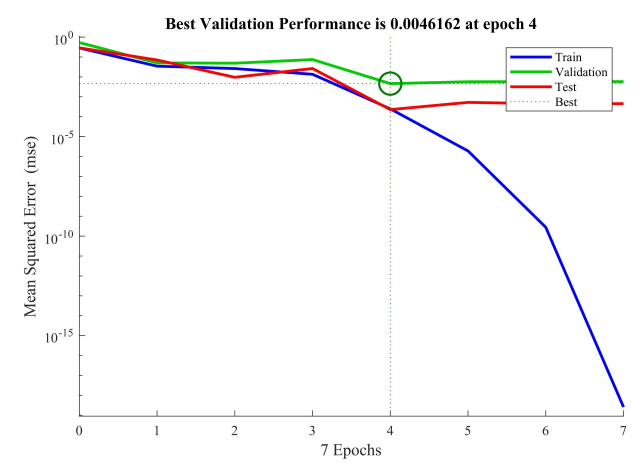

(a)

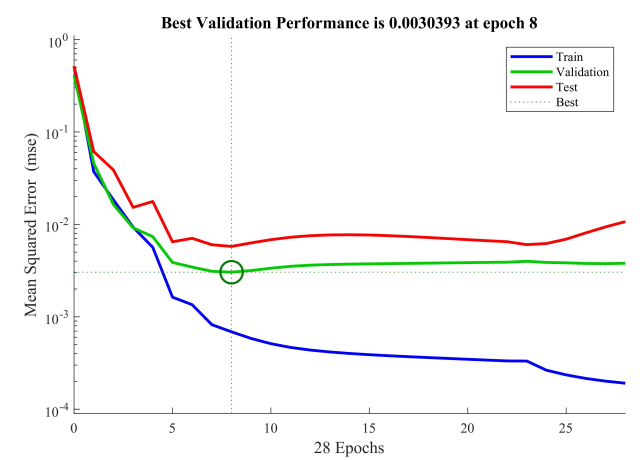

(c)

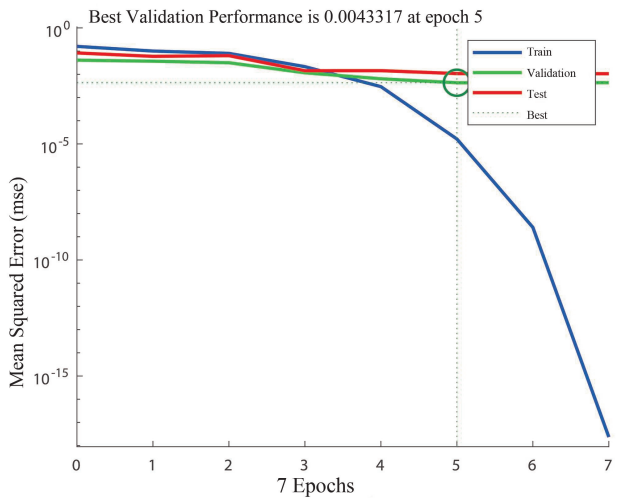

(b)

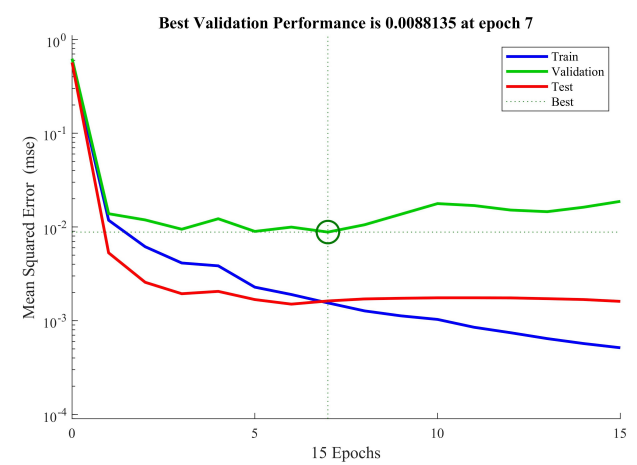

(d)

Figure 4. Cont. 


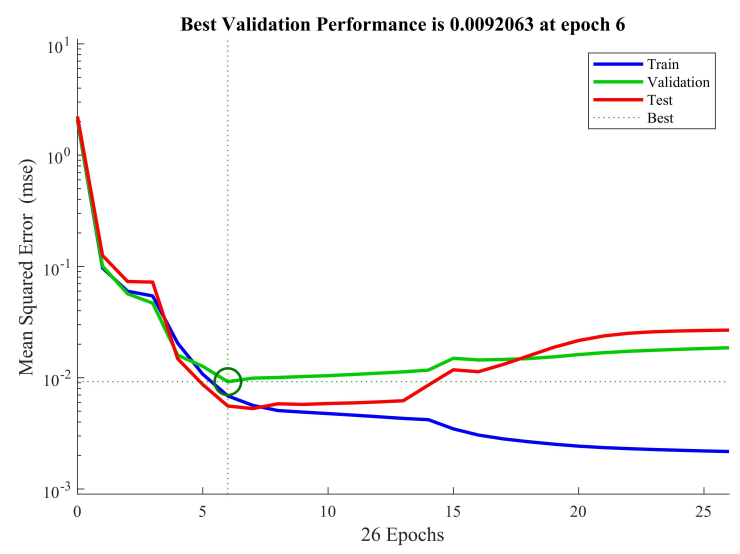

(e)

Figure 4. Performance function of the proposed network. (a) SPSW; Stiffness, (b) SPSW; Strength, (c) RCSW; Strength, (d) RCSW; Stiffness, (e) RCSW; Drift.

Furthermore, Figure 5 illustrates the quality of the estimation as a function of coefficient of determination, R2, in both SPSW and RCSW models. Finally, the MSE values for these data are summarized in Table 5. The proper performance of the network is evident in the estimation of the strength, stiffness and drift ratio of SWs. Therefore, the proposed network can learn the relation between the input and output parameters, and provide the results with appropriate accuracy. Finally, the true values of the strength, stiffness and drift ratio from experimental data and the predictive ANN-based meta-model are compared in Figure 6.
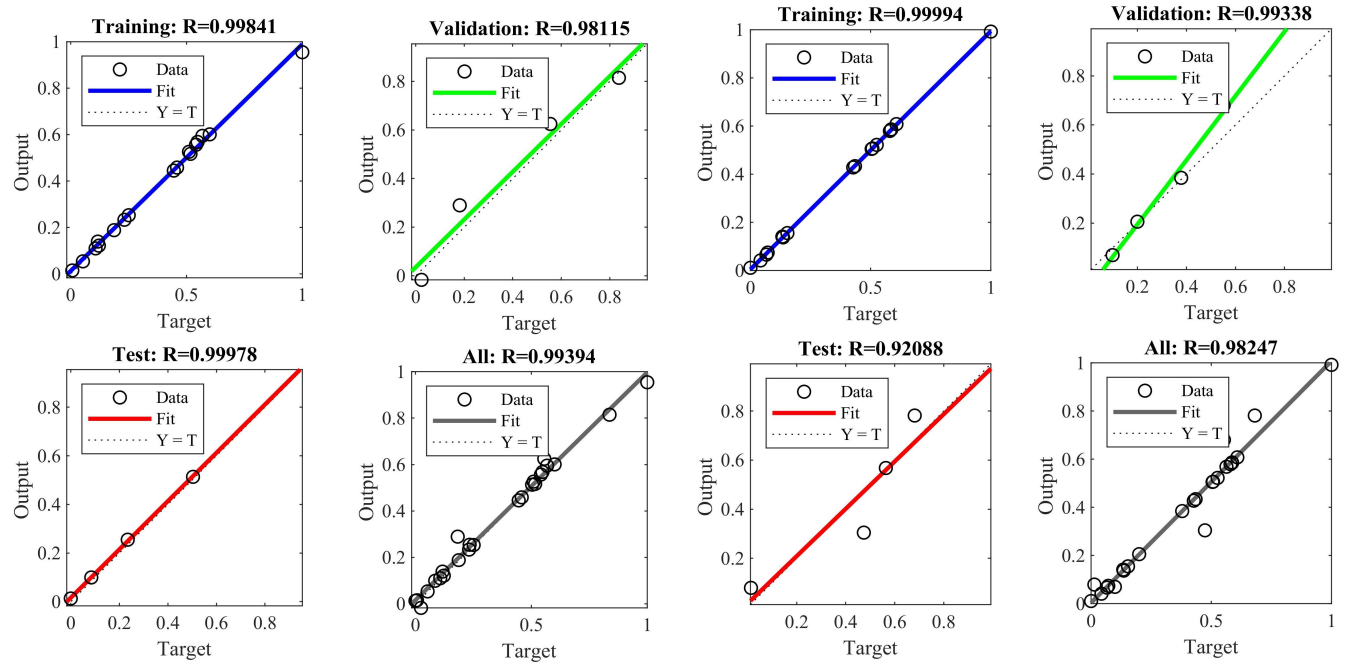

(a)

(b)

Figure 5. Cont. 

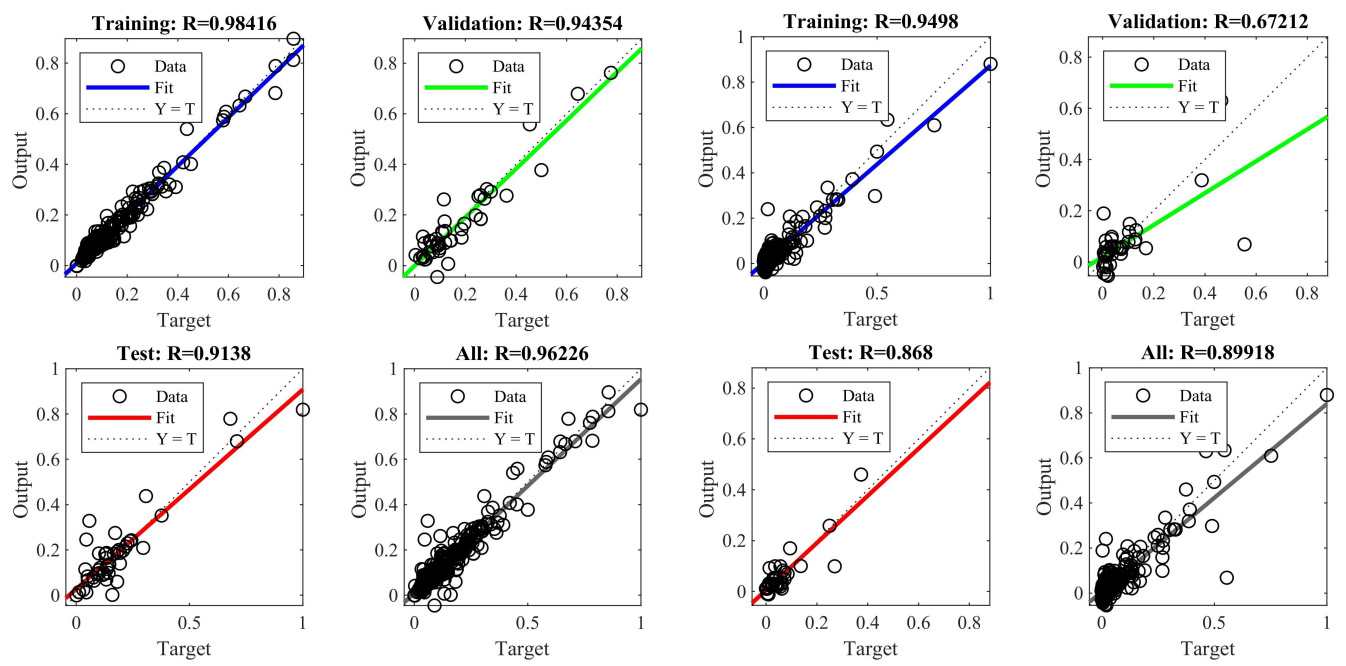

(c)

(d)
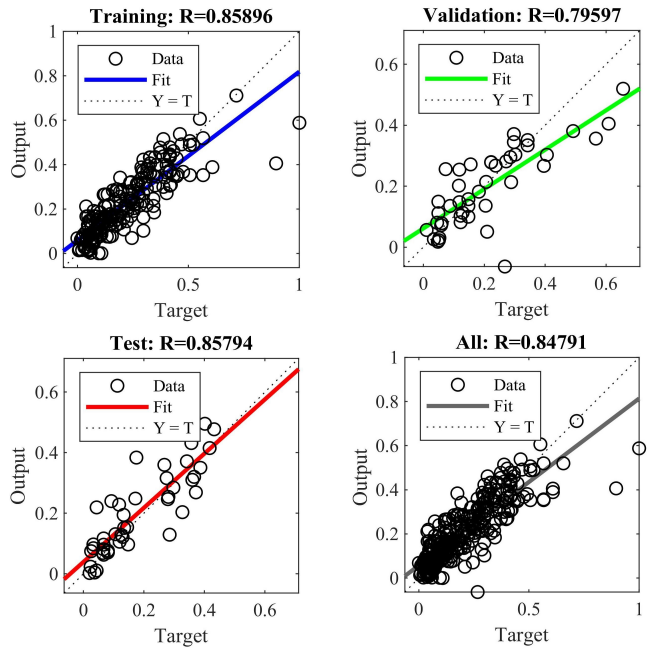

(e)

Figure 5. Regression values for the proposed ANN meta-model. (a) SPSW; Strength, (b) SPSW; Stiffness, (c) RCSW; Strength, (d) RCSW; Stiffness, (e) RCSW; Drift ratio.

Table 5. MSE values for training, validation and test data.

\begin{tabular}{cccc}
\hline Output & Train & Validation & Test \\
\hline Strength of SPSW & $2.49 \times 10^{-4}$ & 0.0046 & $2.28 \times 10^{-4}$ \\
Stiffness of SPSW & $1.64 \times 10^{-5}$ & 0.0043 & 0.0108 \\
Strength of RCSW & 0.00743 & 0.0034 & 0.00123 \\
Stiffness of RCSW & $6.45 \times 10^{-4}$ & 0.0038 & 0.0061 \\
Drift ratio of RCSW & 0.0069 & 0.0059 & 0.0056 \\
\hline
\end{tabular}




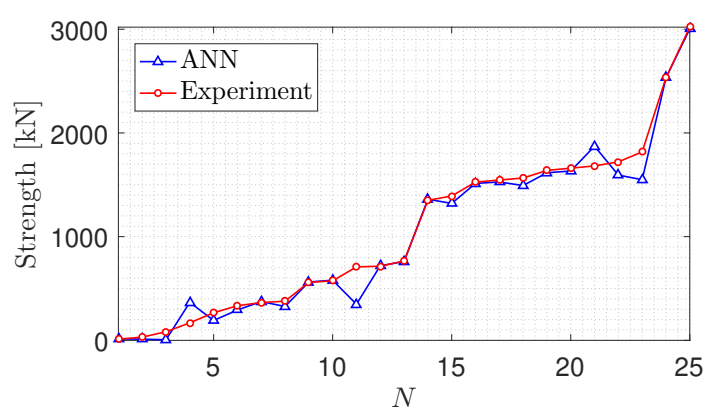

(a)

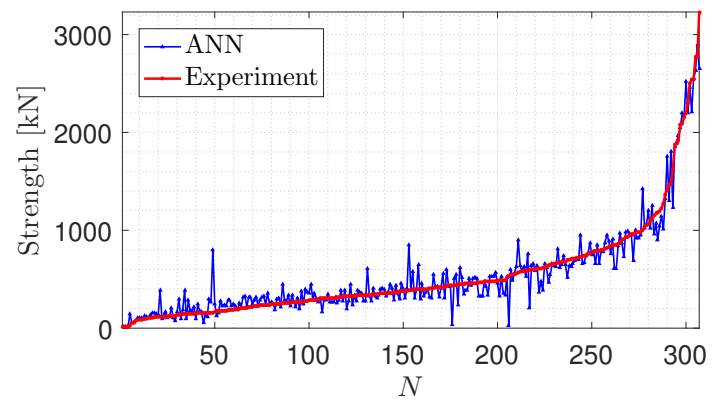

(c)

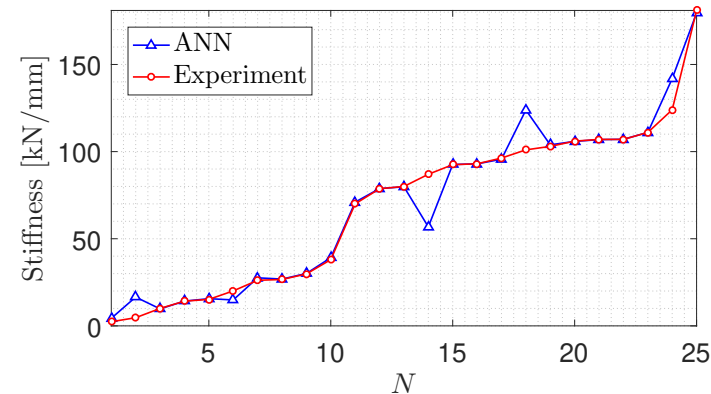

(b)

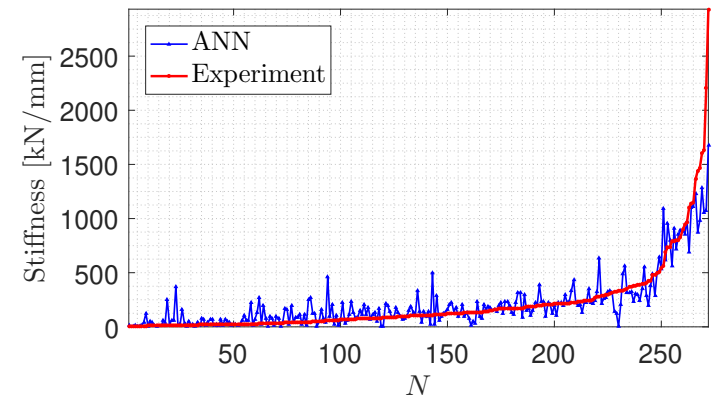

(d)

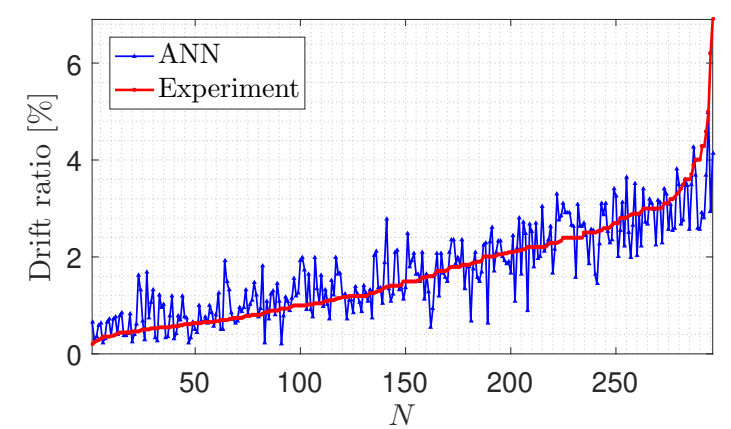

(e)

Figure 6. Comparison of measured experiments and predicted ANN meta-model; Note: sorted in ascending order based on experimental data. (a) SPSW; Strength, (b) SPSW; Stiffness, (c) RCSW; Strength, (d) RCSW; Stiffness, (e) RCSW; Drift.

Thus far, a detailed performance is discussed with respect to MSE. However, one may evaluate the predictive meta-models using other statistical indicators such as root mean square error (RMSE), Nash-Sutcliffe efficiency (NSE) coefficient, mean absolute error (MAE), and correlation coefficient (R). These metrics can be computed using Equation (2).

$$
\begin{aligned}
& \text { RMSE }=\sqrt{\frac{\sum(\hat{y}-y)^{2}}{N}} \quad \text { NSE }=1-\frac{\sum(\hat{y}-y)^{2}}{\sum(y-\bar{y})^{2}} \\
& \mathrm{MAE}=\frac{100}{N} \sum\left|\frac{y-\bar{y}}{y}\right| \quad \mathrm{R}=\frac{\sum(\hat{y}-\bar{y})(y-\bar{y})}{\sqrt{\sum(\hat{y}-\bar{y})^{2}} \sqrt{\sum(y-\bar{y})^{2}}}
\end{aligned}
$$

Table 6 compares these metrics (including MSE) based on all data points obtained from the networks. As seen, there is a good consistency among those five metrics for five meta-models. For example, higher R corresponds with lower RMSE. 
Table 6. Comparison of five statistical indicators to evaluate the accuracy of meta-models.

\begin{tabular}{lccccc}
\hline & MSE & RMSE & NSE & MAE & R \\
\hline P; SPSW & 0.0014 & 0.0374 & 0.9796 & 0.0224 & 0.9939 \\
K; SPSW & 0.0024 & 0.0489 & 0.9632 & 0.022 & 0.9824 \\
P; RCSW & 0.0015 & 0.0389 & 0.9375 & 0.0255 & 0.9622 \\
K; RCSW & 0.0028 & 0.0534 & 0.79 & 0.0278 & 0.8992 \\
$D ;$ RCSW & 0.0067 & 0.082 & 0.7277 & 0.0585 & 0.8479 \\
\hline
\end{tabular}

\subsection{Sensitivity Analysis}

To evaluate the relative importance of the parameters in the network, the Garson's factor was used [78]. The equation provided for the network with a hidden layer is:

$$
Q_{i k}=\frac{\sum_{j=1}^{L}\left(\frac{w_{i j}}{\sum_{r=1}^{N} w_{r j}} v_{j k}\right)}{\sum_{i=1}^{N}\left(\sum_{j=1}^{L} \frac{w_{i j}}{\sum_{r=1}^{N} w_{r j}} v_{j k}\right)}
$$

where $\sum_{r=1}^{N} w_{r j}$ is the sum of the connection weights between the $N$ input neurons and the hidden neuron $j$, and $v_{j k}$ is connection weight between the hidden neuron $j$ and the output neuron $k$ [79].

Sensitivity of each parameter is then presented in Figure 7. The first (and the most important) observation is that nearly all the parameters are contributing effectively in the overall performance of the SWs. Among them, the column's moment of inertia and ultimate stress of the infill plate have most dominant effects. Strength and lateral stiffness of a SPSW depend on the development of diagonal tension field in the infill plate. To develop a uniform diagonal tension field, the boundary elements should have enough flexural stiffness to anchor the tension field [80]. As a result, the flexural stiffness of the boundary elements will have the greatest impact on the load bearing capacity (as seen in this figure). Due to the yielding of the infill plate during loading, its ultimate stress also affects the behavior of the SPSW. Moreover, the thickness and width of the infill plate have significant contribution in the strength. Therefore, the proposed ANN can accurately estimate the behavior of SPSWs. On the other hand, the most important parameters affecting the strength, stiffness and drift ratio of RCSWs are the wall's length (effectiveness $=17.8 \%$ ), the wall's height (effectiveness $=14.6 \%$ ), and the horizontal column's reinforcement ratio (effectiveness $=10.3 \%$ ).

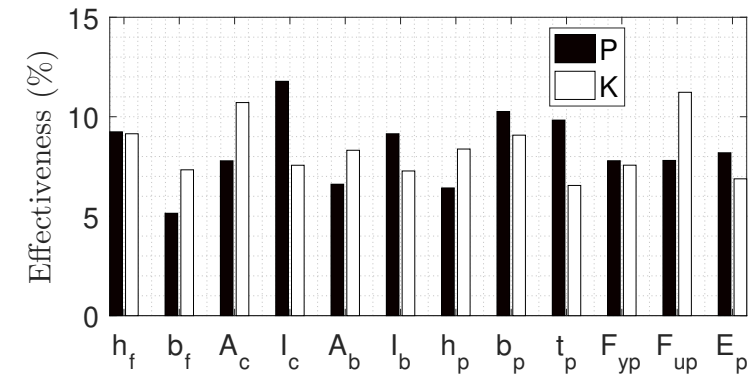

(a)

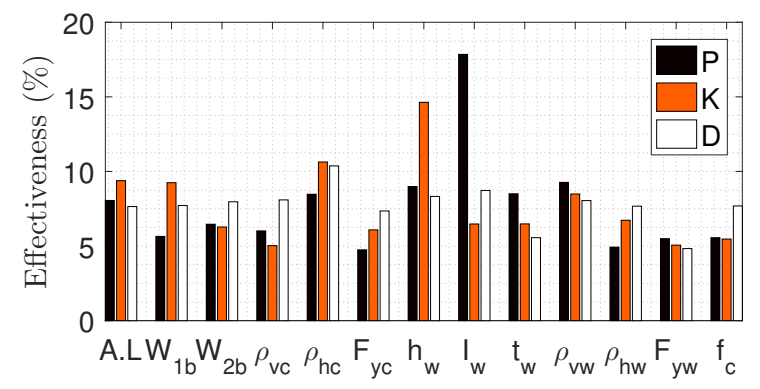

(b)

Figure 7. Relative importance of the input parameters in shear wall meta-model. (a) SPSW, (b) RCSW.

\section{Conclusions}

Understanding the complex behavior of the shear walls through their effective parameters can help to properly determine the lateral response of the structures. In this paper, an efficient computational method was proposed to estimate the strength, stiffness, and drift ratio of the steel plate and reinforced concrete shear walls from a rich library of experimental data. Over 100 papers and reports were synthesized and the available information were extracted. The parameters were mainly related to the 
geometry of the shear walls (such as height, width and thickness of the plate and its surrendering frame), as well as the applied loads, and the material properties. On the other hand, the output was only considered the global behavior of the system (in terms of the stiffness, strength and drift). One may notice that the reinforcement detailing, collapse modes, and buckling parameters were not considered in this meta-modeling. The main reason can be attributed to unavailability of those information in majority of cases. In addition, the failure mode and crack pattern had more qualitative nature, and it was difficult (if not impossible) to present them in a quantitative format (using damage index concept). An artificial neural network was proposed to solve the problem. In this network, the back propagation algorithm was adopted. The network of a single hidden layer with 6 and 10 neurons would have the best performance for SPSW and RCSW, respectively. The major conclusions can be summarized as follows:

- The MSE of test data to estimate the strength and stiffness of the SPSW was 0.000227 and 0.0108 , respectively, which indicate the proper performance of the network.

- The MSE of test data in RCSW was 0.0012, 0.0061, and 0.0056 for strength, stiffness and drift ratio, respectively.

- Sensitivity analysis was performed to determine the relative importance of the input parameters on the shear wall's behavior. It was observed that the stiffness of the vertical boundary elements and the ultimate stresses of the infill plate had largest effect on the strength and stiffness of the SPSW, respectively.

- On the other hand, the most important parameters affecting the strength, stiffness and drift ratio of RCSWs were wall's length, wall's height, and the horizontal column's reinforcement ratio, respectively.

- The performance of the network based on different statistical indicators was also found to be very close.

Finally, the detailed values of the ANN weights are provided (see Appendix B). The proposed network can be used to design new walls, retrofit the existing ones, and validate the finite element models. In design level, the proposed network can provide an estimation of the ultimate strength and stiffness values, which can be further correlated with codified values. This is beyond the conventional design philosophy, which is based on linear elastic models. On the other hand, the performance of existing shear walls can be controlled with this meta-model to make sure they have a safe/proper behavior under the target loads, and, if not, they can be retrofitted.

Author Contributions: Conceptualization, M.J.M. and M.A.H.-A.; methodology, M.J.M. and M.A.H.-A.; software, M.J.M.; validation, M.J.M.; formal analysis, M.J.M.; investigation, M.J.M. and M.A.H.-A.; resources, M.J.M. and M.A.H.-A.; data curation, M.J.M.; writing—original draft preparation, M.J.M.; writing—review and editing, M.A.H.-A.; visualization, M.J.M. and M.A.H.-A.; supervision, M.A.H.-A.; project administration, M.A.H.-A.; funding acquisition, M.A.H.-A.

Funding: This research received no external funding.

Acknowledgments: The author would like to thank the editor and reviewers for their helpful and constructive comments that greatly contributed to improving the final version of the paper.

Conflicts of Interest: The authors declare no conflict of interest. 
Appendix A. Details of Shear Wall Library

Table A1. Library of steel plate shear walls information.

\begin{tabular}{|c|c|c|c|c|c|c|c|c|c|c|c|c|c|c|}
\hline Reference & $\begin{array}{c}\mathrm{hf} \\
\mathrm{mm}\end{array}$ & $\begin{array}{c}\text { bf } \\
\text { mm }\end{array}$ & $\begin{array}{c}\text { Ac } \\
\text { mm }^{2}\end{array}$ & $\begin{array}{c}\text { Ic } \\
\text { mm }^{4}\end{array}$ & $\begin{array}{c}\mathrm{Ab} \\
\mathbf{m m}^{2}\end{array}$ & $\begin{array}{c}\mathrm{Ib} \\
\text { mm }^{4}\end{array}$ & $\begin{array}{l}\mathrm{hp} \\
\mathrm{mm}\end{array}$ & $\begin{array}{c}\text { bp } \\
\text { mm }\end{array}$ & $\underset{\mathrm{mm}}{\mathrm{tp}}$ & $\begin{array}{c}\text { Fyp } \\
\text { Pa }\end{array}$ & $\begin{array}{c}\text { Fup } \\
\text { Pa }\end{array}$ & $\begin{array}{l}\text { Ep } \\
\text { Pa }\end{array}$ & $\begin{array}{l}\mathbf{P} \\
\mathbf{N}\end{array}$ & $\begin{array}{c}\mathrm{K} \\
\mathrm{N} / \mathrm{mm}\end{array}$ \\
\hline Sigariyazd et al. [81] & 1100 & 1400 & $7.05 \times 10^{3}$ & $3.86 \times 10^{7}$ & $4.10 \times 10^{3}$ & $1.13 \times 10^{7}$ & 1020 & 1200 & 1.50 & $2.22 \times 10^{8}$ & $3.15 \times 10^{8}$ & $2.00 \times 10^{11}$ & $7.15 \times 10^{5}$ & $9.63 \times 10^{4}$ \\
\hline Shekastehband et al. [82] & 620 & 620 & $3.40 \times 10^{3}$ & $8.64 \times 10^{5}$ & $3.40 \times 10^{3}$ & $8.64 \times 10^{5}$ & 500 & 460 & 0.50 & $1.38 \times 10^{8}$ & $2.81 \times 10^{8}$ & $2.00 \times 10^{11}$ & $1.15 \times 10^{4}$ & $2.38 \times 10^{3}$ \\
\hline Shekastehband et al. [82] & 620 & 620 & $3.40 \times 10^{3}$ & $8.64 \times 10^{5}$ & $3.40 \times 10^{3}$ & $8.64 \times 10^{5}$ & 500 & 460 & 1.25 & $1.82 \times 10^{8}$ & $3.28 \times 10^{8}$ & $2.00 \times 10^{11}$ & $3.13 \times 10^{4}$ & $4.69 \times 10^{3}$ \\
\hline Berman et al. [83] & 1830 & 3660 & $1.82 \times 10^{4}$ & $3.47 \times 10^{8}$ & $1.63 \times 10^{4}$ & $6.37 \times 10^{8}$ & 1350 & 3340 & 1.00 & $2.15 \times 10^{8}$ & $4.66 \times 10^{8}$ & $1.60 \times 10^{11}$ & $3.64 \times 10^{5}$ & $1.06 \times 10^{5}$ \\
\hline Yu et al. [84] & 1250 & 1350 & $5.12 \times 10^{3}$ & $2.88 \times 10^{7}$ & $2.72 \times 10^{3}$ & $1.84 \times 10^{7}$ & 1050 & 1180 & 5.00 & $3.15 \times 10^{8}$ & $4.78 \times 10^{8}$ & $2.00 \times 10^{11}$ & $7.08 \times 10^{5}$ & $2.98 \times 10^{4}$ \\
\hline Choi and Park [85] & 1075 & 1650 & $9.90 \times 10^{3}$ & $5.53 \times 10^{7}$ & $5.80 \times 10^{3}$ & $3.25 \times 10^{7}$ & 1000 & 1500 & 4.00 & $2.40 \times 10^{8}$ & $4.00 \times 10^{8}$ & $2.00 \times 10^{11}$ & $1.39 \times 10^{6}$ & $7.00 \times 10^{4}$ \\
\hline Choi and Park [85] & 1075 & 2350 & $9.90 \times 10^{3}$ & $5.53 \times 10^{7}$ & $5.80 \times 10^{3}$ & $3.25 \times 10^{7}$ & 1000 & 2200 & 4.00 & $2.40 \times 10^{8}$ & $4.00 \times 10^{8}$ & $2.00 \times 10^{11}$ & $1.82 \times 10^{6}$ & $1.07 \times 10^{5}$ \\
\hline Choi and Park [85] & 1075 & 2350 & $7.20 \times 10^{3}$ & $2.40 \times 10^{7}$ & $5.80 \times 10^{3}$ & $3.25 \times 10^{7}$ & 1000 & 2200 & 4.00 & $2.40 \times 10^{8}$ & $4.00 \times 10^{8}$ & $2.00 \times 10^{11}$ & $1.57 \times 10^{6}$ & $1.03 \times 10^{5}$ \\
\hline Wang et al. [86] & 1100 & 2050 & $9.40 \times 10^{3}$ & $7.15 \times 10^{7}$ & $6.35 \times 10^{3}$ & $4.72 \times 10^{7}$ & 1000 & 1800 & 6.00 & $4.18 \times 10^{8}$ & $5.63 \times 10^{8}$ & $1.98 \times 10^{11}$ & $1.66 \times 10^{6}$ & $1.07 \times 10^{5}$ \\
\hline Wang et al. [86] & 1100 & 2400 & $8.10 \times 10^{3}$ & $9.77 \times 10^{7}$ & $6.35 \times 10^{3}$ & $4.72 \times 10^{7}$ & 1000 & 2160 & 6.00 & $4.18 \times 10^{8}$ & $5.63 \times 10^{8}$ & $1.98 \times 10^{11}$ & $1.55 \times 10^{6}$ & $9.25 \times 10^{4}$ \\
\hline Wang et al. [86] & 100 & 2400 & $8.10 \times 10^{3}$ & $9.77 \times 10^{7}$ & $6.35 \times 10^{3}$ & $4.72 \times 10^{7}$ & 1000 & 2400 & 6.00 & $4.18 \times 10^{8}$ & $5.63 \times 10^{8}$ & $1.98 \times 10^{11}$ & $1.64 \times 10^{6}$ & $7.87 \times 10^{4}$ \\
\hline Sabouri-Ghomi and Sajjadi [87] & 1105 & 1500 & $5.40 \times 10^{3}$ & $6.34 \times 10^{6}$ & $1.06 \times 10^{4}$ & $1.28 \times 10^{8}$ & 960 & 1410 & 2.00 & $1.92 \times 10^{8}$ & $2.77 \times 10^{8}$ & $2.00 \times 10^{11}$ & $5.74 \times 10^{5}$ & $1.81 \times 10^{5}$ \\
\hline Chen and Jhang [88] & 1500 & 1500 & $8.34 \times 10^{3}$ & $1.33 \times 10^{7}$ & $8.34 \times 10^{3}$ & $1.33 \times 10^{7}$ & 1200 & 1200 & 15.00 & $8.54 \times 10^{7}$ & $2.58 \times 10^{8}$ & $1.75 \times 10^{11}$ & $1.35 \times 10^{6}$ & N.A. \\
\hline Chen and Jhang [88] & 1500 & 1500 & $8.34 \times 10^{3}$ & $1.33 \times 10^{7}$ & $8.34 \times 10^{3}$ & $1.33 \times 10^{7}$ & 1200 & 1200 & 8.00 & $9.28 \times 10^{7}$ & $2.72 \times 10^{8}$ & $1.75 \times 10^{11}$ & $5.59 \times 10^{5}$ & N.A. \\
\hline Nateghi-Alahi and Khazaei-Poul [89] & 500 & 500 & $2.70 \times 10^{3}$ & $4.12 \times 10^{6}$ & $2.70 \times 10^{3}$ & $4.12 \times 10^{6}$ & 380 & 3800 & 0.90 & $1.97 \times 10^{8}$ & $3.23 \times 10^{8}$ & $2.04 \times 10^{11}$ & $8.26 \times 10^{4}$ & $2.62 \times 10^{4}$ \\
\hline Caccese et al. [90] & 838 & 1245 & $2.48 \times 10^{3}$ & $4.70 \times 10^{6}$ & $1.08 \times 10^{3}$ & $1.05 \times 10^{6}$ & 762 & 1140 & 0.76 & $3.06 \times 10^{8}$ & $3.47 \times 10^{8}$ & $1.75 \times 10^{11}$ & $1.69 \times 10^{5}$ & $1.42 \times 10^{4}$ \\
\hline Caccese et al. [90] & 1245 & 2477 & $4.70 \times 10^{6}$ & $1.08 \times 10^{3}$ & $1.05 \times 10^{6}$ & $7.62 \times 10^{2}$ & 1140 & 1140 & 1.90 & $2.91 \times 10^{8}$ & $3.15 \times 10^{8}$ & $1.75 \times 10^{11}$ & $3.34 \times 10^{5}$ & $2.01 \times 10^{4}$ \\
\hline Caccese et al. [90] & 1245 & 2477 & $4.70 \times 10^{6}$ & $1.08 \times 10^{3}$ & $1.05 \times 10^{6}$ & $7.62 \times 10^{2}$ & 1140 & 1140 & 2.66 & $2.95 \times 10^{8}$ & $4.04 \times 10^{8}$ & $1.75 \times 10^{11}$ & $3.78 \times 10^{5}$ & $2.66 \times 10^{4}$ \\
\hline Alavi and Nateghi [91] & 1366 & 1960 & $5.43 \times 10^{3}$ & $2.49 \times 10^{7}$ & $5.43 \times 10^{3}$ & $2.49 \times 10^{7}$ & 1102 & 1720 & 0.80 & $2.80 \times 10^{8}$ & $5.00 \times 10^{8}$ & $2.04 \times 10^{11}$ & $7.65 \times 10^{5}$ & $1.52 \times 10^{4}$ \\
\hline Park et al. [92] & 1100 & 2250 & $1.50 \times 10^{4}$ & $2.09 \times 10^{8}$ & $9.60 \times 10^{3}$ & $8.55 \times 10^{7}$ & 1500 & 1000 & 2.00 & $2.40 \times 10^{8}$ & $4.00 \times 10^{8}$ & $1.49 \times 10^{11}$ & $1.72 \times 10^{6}$ & $8.70 \times 10^{4}$ \\
\hline Park et al. [92] & 1100 & 2250 & $1.50 \times 10^{4}$ & $2.09 \times 10^{8}$ & $9.60 \times 10^{3}$ & $8.55 \times 10^{7}$ & 1500 & 1000 & 4.00 & $3.30 \times 10^{8}$ & $4.90 \times 10^{8}$ & $1.49 \times 10^{11}$ & $2.53 \times 10^{6}$ & $1.11 \times 10^{5}$ \\
\hline Park et al. [92] & 1100 & 2250 & $1.50 \times 10^{4}$ & $2.09 \times 10^{8}$ & $9.60 \times 10^{3}$ & $8.55 \times 10^{7}$ & 1500 & 1000 & 6.00 & $3.30 \times 10^{8}$ & $4.90 \times 10^{8}$ & $1.49 \times 10^{11}$ & $3.02 \times 10^{6}$ & $1.24 \times 10^{5}$ \\
\hline Park et al. [92] & 1100 & 2250 & $8.25 \times 10^{3}$ & $1.15 \times 10^{8}$ & $9.60 \times 10^{3}$ & $8.55 \times 10^{7}$ & 1500 & 1000 & 4.00 & $3.30 \times 10^{8}$ & $4.90 \times 10^{8}$ & $1.49 \times 10^{11}$ & $1.53 \times 10^{6}$ & $9.30 \times 10^{4}$ \\
\hline Park et al. [92] & 1100 & 2250 & $8.25 \times 10^{3}$ & $1.15 \times 10^{8}$ & $9.60 \times 10^{3}$ & $8.55 \times 10^{7}$ & 1500 & 1000 & 6.00 & $3.30 \times 10^{8}$ & $4.90 \times 10^{8}$ & $1.49 \times 10^{11}$ & $1.68 \times 10^{6}$ & $1.01 \times 10^{5}$ \\
\hline Lubell et al. [93] & 900 & 900 & $1.07 \times 10^{3}$ & $1.04 \times 10^{6}$ & $1.07 \times 10^{3}$ & $1.04 \times 10^{6}$ & 800 & 800 & 1.50 & $3.20 \times 10^{8}$ & N.A. & N.A. & $2.66 \times 10^{5}$ & $3.80 \times 10^{4}$ \\
\hline Roberts and Sabouri-Ghomi [94] & 370 & 370 & N.A. & N.A. & N.A. & N.A. & 300 & 300 & 0.83 & $2.19 \times 10^{8}$ & $3.28 \times 10^{8}$ & $2.02 \times 10^{11}$ & $5.17 \times 10^{4}$ & N.A. \\
\hline Roberts and Sabouri-Ghomi [94] & 370 & 370 & N.A. & N.A. & N.A. & N.A. & 300 & 300 & 1.23 & $1.52 \times 10^{8}$ & $2.28 \times 10^{6}$ & $2.03 \times 10^{11}$ & $6.83 \times 10^{4}$ & N.A. \\
\hline Roberts and Sabouri-Ghomi [94] & 370 & 370 & N.A. & N.A. & N.A. & N.A. & 300 & 450 & 0.83 & $2.19 \times 10^{8}$ & $3.28 \times 10^{8}$ & $2.02 \times 10^{11}$ & $6.25 \times 10^{4}$ & N.A. \\
\hline Roberts and Sabouri-Ghomi [94] & 370 & 370 & N.A. & N.A. & N.A. & N.A. & 300 & 450 & 1.23 & $1.52 \times 10^{8}$ & $2.28 \times 10^{6}$ & $2.03 \times 10^{11}$ & $7.52 \times 10^{4}$ & N.A. \\
\hline
\end{tabular}


Table A2. Library of reinforced concrete shear walls information.

\begin{tabular}{|c|c|c|c|c|c|c|c|c|c|c|c|c|c|c|c|c|}
\hline Reference & $\begin{array}{l}\text { A.L } \\
\text { kN }\end{array}$ & $\begin{array}{c}\text { ac } \\
\mathrm{mm}\end{array}$ & $\begin{array}{c}\mathrm{bc} \\
\mathrm{mm}\end{array}$ & $\begin{array}{c}\rho_{v c} \\
\%\end{array}$ & $\begin{array}{c}\rho_{h c} \\
\%\end{array}$ & $\begin{array}{l}\text { Fyc } \\
\text { MPa }\end{array}$ & $\begin{array}{l}\text { hw } \\
\text { mm }\end{array}$ & $\begin{array}{l}\mathrm{lw} \\
\mathrm{mm}\end{array}$ & $\begin{array}{c}\mathrm{tw} \\
\mathrm{mm}\end{array}$ & $\underset{\%}{\rho_{v w}}$ & $\underset{\%}{\rho_{h w}}$ & $\begin{array}{l}\text { Fyw } \\
\text { MPa }\end{array}$ & $\begin{array}{l}\text { Fcw } \\
\text { MPa }\end{array}$ & $\begin{array}{c}P \\
k N\end{array}$ & $\begin{array}{c}\mathrm{K} \\
\mathrm{kN} / \mathrm{mm}\end{array}$ & $\begin{array}{l}\mathrm{D} \\
\%\end{array}$ \\
\hline Carrillo et al. [95] & 60 & 102 & 102 & 0.670 & 0.420 & 434.0 & 2426 & 2402 & 102 & 0.140 & 0.140 & 447.0 & 18.80 & 408.0 & N.A. & 2.01 \\
\hline Carrillo et al. [95] & 60 & 101 & 101 & 0.980 & 0.420 & 430.0 & 2426 & 2402 & 101 & 0.280 & 0.280 & 447.0 & 18.80 & 617.0 & N.A. & 1.71 \\
\hline Carrillo et al. [95] & 60 & 102 & 102 & 0.680 & 0.420 & 434.0 & 2423 & 2399 & 102 & 0.140 & 0.140 & 447.0 & 17.50 & 352.0 & N.A. & 1.03 \\
\hline Carrillo et al. [95] & 60 & 101 & 101 & 0.980 & 0.420 & 430.0 & 2421 & 2397 & 101 & 0.280 & 0.280 & 447.0 & 17.50 & 453.0 & N.A. & 1.72 \\
\hline Carrillo et al. [95] & 60 & 100 & 100 & 0.220 & 0.430 & 443.0 & 2430 & 5400 & 100 & 0.280 & 0.280 & 447.0 & 16.20 & 766.0 & N.A. & 1.05 \\
\hline Carrillo et al. [95] & 60 & 103 & 103 & 0.220 & 0.410 & 456.0 & 2430 & 5396 & 103 & 0.120 & 0.120 & 605.0 & 20.00 & 776.0 & N.A. & 0.45 \\
\hline Carrillo et al. [95] & 60 & 103 & 103 & 0.720 & 0.420 & 443.0 & 2422 & 2398 & 103 & 0.120 & 0.120 & 605.0 & 20.00 & 329.0 & N.A. & 0.54 \\
\hline Carrillo et al. [95] & 60 & 101 & 101 & 0.960 & 0.420 & 443.0 & 2478 & 1239 & 101 & 0.120 & 0.120 & 605.0 & 20.00 & 154.0 & N.A. & 0.68 \\
\hline Carrillo et al. [95] & 60 & 83 & 83 & 0.780 & 0.430 & 411.0 & 1916 & 1916 & 83 & 0.110 & 0.110 & 630.0 & 24.70 & 234.0 & 68.6 & 0.54 \\
\hline Carrillo et al. [95] & 60 & 84 & 84 & 1.020 & 0.420 & 411.0 & 1921 & 1921 & 84 & 0.260 & 0.260 & 435.0 & 24.70 & 274.0 & 72 & 1.51 \\
\hline Yuan et al. [96] & 1527 & 200 & 200 & 0.370 & 0.800 & 473.0 & 2360 & 1280 & 200 & 0.390 & 0.330 & 544.0 & 45.90 & 656.0 & N.A. & 1.14 \\
\hline Yuan et al. [96] & 1527 & 200 & 200 & 0.370 & 0.800 & 473.0 & 2360 & 1280 & 200 & 0.710 & 0.710 & 544.0 & 45.90 & 740.0 & N.A. & 1.15 \\
\hline Shiga et al. [97] & 0 & 150 & 120 & 0.049 & 0.089 & 390.0 & 600 & 1000 & 50 & 0.250 & 0.250 & 300.0 & 16.00 & 177.0 & N.A. & 2.40 \\
\hline Shiga et al. [97] & 0 & 150 & 120 & 0.049 & 0.089 & 390.0 & 600 & 1000 & 50 & 0.250 & 0.250 & 300.0 & 16.00 & 188.0 & N.A. & 2.40 \\
\hline Shiga et al. [97] & 0 & 150 & 120 & 0.049 & 0.089 & 390.0 & 600 & 1000 & 50 & 0.250 & 0.250 & 300.0 & 16.00 & 211.0 & N.A. & 2.40 \\
\hline Shiga et al. [97] & 0 & 150 & 120 & 0.049 & 0.089 & 390.0 & 600 & 1000 & 50 & 0.250 & 0.250 & 300.0 & 16.00 & 231.0 & N.A. & 4.28 \\
\hline Shiga et al. [97] & 200 & 150 & 120 & 0.049 & 0.089 & 390.0 & 600 & 1000 & 50 & 0.250 & 0.250 & 300.0 & 16.00 & 341.0 & N.A. & 4.28 \\
\hline Shiga et al. [97] & 0 & 150 & 120 & 0.049 & 0.089 & 390.0 & 600 & 1000 & 50 & 0.500 & 0.500 & 300.0 & 16.00 & 240.0 & N.A. & 2.34 \\
\hline Shiga et al. [97] & 200 & 150 & 120 & 0.049 & 0.089 & 390.0 & 600 & 1000 & 50 & 0.500 & 0.500 & 300.0 & 16.00 & 310.0 & N.A. & 2.32 \\
\hline Shiga et al. [97] & 400 & 150 & 120 & 0.049 & 0.089 & 390.0 & 600 & 1000 & 50 & 0.500 & 0.500 & 300.0 & 16.00 & 287.0 & N.A. & 2.40 \\
\hline Greifenhagen and Lestuzzi [98] & 136 & 100 & 100 & 0.300 & 0.300 & 504.0 & 610 & 1000 & 100 & 0.300 & 0.300 & 504.0 & 50.70 & 204.0 & 333 & 3.50 \\
\hline Greifenhagen and Lestuzzi [98] & 136 & 100 & 100 & 0.300 & 0.000 & 504.0 & 610 & 1000 & 100 & 0.300 & 0.000 & 504.0 & 51.00 & 203.0 & 111 & 2.50 \\
\hline Greifenhagen and Lestuzzi [98] & 136 & 80 & 80 & 0.300 & 0.300 & 745.0 & 610 & 900 & 80 & 0.300 & 0.300 & 745.0 & 20.10 & 176.0 & 108 & 1.70 \\
\hline Greifenhagen and Lestuzzi [98] & 136 & 80 & 80 & 0.300 & 0.300 & 745.0 & 610 & 900 & 80 & 0.300 & 0.300 & 745.0 & 24.40 & 135.0 & 125 & 2.00 \\
\hline Looi et al. [99] & 220 & 80 & 80 & 2.000 & 1.400 & 601.0 & 800 & 800 & 80 & 2.000 & 1.400 & 601.0 & 29.10 & 252.5 & 170 & 1.11 \\
\hline Looi et al. [99] & 380 & 80 & 80 & 2.000 & 1.400 & 601.0 & 800 & 800 & 80 & 2.000 & 1.400 & 601.0 & 26.40 & 245.0 & 160 & 1.03 \\
\hline Looi et al. [99] & 680 & 80 & 80 & 2.000 & 1.400 & 601.0 & 800 & 800 & 80 & 2.000 & 1.400 & 601.0 & 27.60 & 249.3 & 171 & 0.63 \\
\hline Looi et al. [99] & 780 & 80 & 80 & 2.000 & 1.400 & 601.0 & 800 & 800 & 80 & 2.000 & 1.400 & 601.0 & 28.00 & 250.9 & 200 & 0.47 \\
\hline Lopes [100] & 96 & 45 & 115 & 4.120 & 0.800 & 436.0 & 495 & 450 & 45 & 0.620 & 0.400 & 414.0 & 45.00 & 108.3 & 14.4 & 2.12 \\
\hline Lopes [100] & 70 & 45 & 115 & 4.120 & 5.000 & 436.0 & 495 & 450 & 45 & 0.620 & 2.800 & 414.0 & 44.50 & 80.3 & 19.6 & 1.65 \\
\hline Lopes [100] & 80 & 45 & 115 & 4.120 & 4.000 & 436.0 & 495 & 450 & 45 & 0.620 & 2.000 & 414.0 & 45.10 & 83.6 & 17.5 & 2.52 \\
\hline Yanez et al. [101] & 0 & 120 & 120 & 0.500 & 0.400 & 475.0 & 2300 & 2000 & 120 & 0.500 & 0.400 & 475.0 & 34.00 & 287.0 & 37.5 & 6.20 \\
\hline Bouchon et al. [102] & 150 & 180 & 100 & 2.500 & 0.070 & 565.0 & 750 & 1500 & 100 & 0.300 & 0.300 & 585.0 & 31.70 & 550.0 & N.A. & N.A \\
\hline Bouchon et al. [102] & 150 & 180 & 100 & 2.500 & 0.070 & 565.0 & 750 & 1500 & 100 & 0.500 & 0.500 & 615.0 & 36.40 & 645.0 & N.A. & N.A \\
\hline
\end{tabular}


Table A2. Cont.

\begin{tabular}{|c|c|c|c|c|c|c|c|c|c|c|c|c|c|c|c|c|}
\hline Reference & $\begin{array}{l}\text { A.L } \\
\text { kN }\end{array}$ & $\begin{array}{c}\text { ac } \\
\mathrm{mm}\end{array}$ & $\begin{array}{l}\mathrm{bc} \\
\mathrm{mm}\end{array}$ & $\begin{array}{c}\rho_{v c} \\
\%\end{array}$ & $\begin{array}{c}\rho_{h c} \\
\%\end{array}$ & $\begin{array}{l}\text { Fyc } \\
\text { MPa }\end{array}$ & $\begin{array}{l}\text { hw } \\
\text { mm }\end{array}$ & $\begin{array}{l}\mathrm{lw} \\
\mathrm{mm}\end{array}$ & $\begin{array}{c}\mathrm{tw} \\
\mathrm{mm}\end{array}$ & $\underset{\%}{\rho_{v w}}$ & $\underset{\%}{\rho_{h w}}$ & $\begin{array}{l}\text { Fyw } \\
\text { MPa }\end{array}$ & $\begin{array}{l}\text { Fcw } \\
\text { MPa }\end{array}$ & $\begin{array}{c}\mathbf{P} \\
\mathbf{k N}\end{array}$ & $\begin{array}{c}\mathrm{K} \\
\mathrm{kN} / \mathrm{mm}\end{array}$ & $\begin{array}{l}\mathrm{D} \\
\%\end{array}$ \\
\hline Bouchon et al. [102] & 150 & 180 & 100 & 2.500 & 0.070 & 565.0 & 750 & 1500 & 100 & 0.800 & 0.800 & 620.0 & 28.60 & 853.0 & N.A. & N.A. \\
\hline Tasnimi [103] & 0 & 50 & 80 & 2.800 & 0.250 & 276.0 & 1500 & 500 & 50 & 0.250 & 0.250 & 216.0 & $21.6 \mathrm{cy}$ & 15.4 & 3.29 & 1.10 \\
\hline Tasnimi [103] & 0 & 50 & 80 & 2.800 & 0.250 & 276.0 & 1500 & 500 & 50 & 0.250 & 0.250 & 216.0 & 21.60 & 19.6 & 4.5 & 0.75 \\
\hline Tasnimi [103] & 0 & 50 & 80 & 2.800 & 0.250 & 276.0 & 1500 & 500 & 50 & 0.250 & 0.250 & 216.0 & 22.45 & 17.5 & 4.1 & 1.03 \\
\hline Tasnimi [103] & 0 & 50 & 80 & 2.800 & 0.250 & 276.0 & 1500 & 500 & 50 & 0.250 & 0.250 & 216.0 & 23.45 & 19.8 & 3.5 & 0.90 \\
\hline Lefas et al. [104] & 0 & 70 & 140 & 3.100 & 1.200 & 470.0 & 750 & 750 & 70 & 2.400 & 1.100 & 470.0 & $52.3 \mathrm{Cu}$ & 260.0 & 102.9 & 1.10 \\
\hline Lefas et al. [104] & 230 & 70 & 140 & 3.100 & 1.200 & 470.0 & 750 & 750 & 70 & 2.400 & 1.100 & 470.0 & 53.60 & 340.0 & 173 & 1.18 \\
\hline Lefas et al. [104] & 355 & 70 & 140 & 3.100 & 1.200 & 470.0 & 750 & 750 & 70 & 2.400 & 1.100 & 470.0 & 40.60 & 330.0 & 135.1 & 1.18 \\
\hline Lefas et al. [104] & 0 & 70 & 140 & 3.100 & 1.200 & 470.0 & 750 & 750 & 70 & 2.400 & 1.100 & 470.0 & 42.10 & 265.0 & 102.9 & 1.49 \\
\hline Lefas et al. [104] & 185 & 70 & 140 & 3.100 & 1.200 & 470.0 & 750 & 750 & 70 & 2.400 & 1.100 & 470.0 & 43.30 & 320.0 & 166.6 & 1.07 \\
\hline Lefas et al. [104] & 460 & 70 & 140 & 3.100 & 1.200 & 470.0 & 750 & 750 & 70 & 2.400 & 1.100 & 470.0 & 51.70 & 355.0 & 200 & 0.77 \\
\hline Lefas et al. [104] & 0 & 70 & 140 & 3.100 & 1.200 & 470.0 & 750 & 750 & 70 & 2.400 & 0.370 & 470.0 & 48.30 & 247.0 & 65 & 1.43 \\
\hline Lefas et al. [104] & 0 & 65 & 140 & 3.300 & 0.900 & 470.0 & 1300 & 650 & 65 & 2.500 & 0.8 & 470.0 & 42.80 & 127.0 & 31 & 1.58 \\
\hline Lefas et al. [104] & 182 & 65 & 140 & 3.300 & 0.900 & 470.0 & 1300 & 650 & 65 & 2.500 & 0.800 & 470.0 & 50.60 & 150.0 & 35.9 & 1.17 \\
\hline Lefas et al. [104] & 343 & 65 & 140 & 3.300 & 0.900 & 470.0 & 1300 & 650 & 65 & 2.500 & 0.800 & 470.0 & 47.80 & 180.0 & 38.4 & 1.01 \\
\hline Lefas et al. [104] & 0 & 65 & 140 & 3.300 & 0.900 & 470.0 & 1300 & 650 & 65 & 2.500 & 0.800 & 470.0 & 48.30 & 120.0 & 34.4 & 1.39 \\
\hline Lefas et al. [104] & 325 & 65 & 140 & 3.300 & 0.900 & 470.0 & 1300 & 650 & 65 & 2.500 & 0.800 & 470.0 & 45.00 & 150.0 & 62.5 & 0.73 \\
\hline Lefas et al. [104] & 0 & 65 & 140 & 3.300 & 0.900 & 470.0 & 1300 & 650 & 65 & 2.500 & 0.400 & 470.0 & 30.10 & 123.0 & 25.6 & 1.61 \\
\hline Lefas and Kotsovos [105] & 0 & 65 & 140 & 3.300 & 0.900 & 470.0 & 1300 & 650 & 65 & 1.500 & 0.350 & 470.0 & 30.10 & 117.7 & 11.4 & 1.61 \\
\hline Lefas and Kotsovos [105] & 0 & 65 & 140 & 3.300 & 0.900 & 470.0 & 1300 & 650 & 65 & 1.500 & 0.350 & 470.0 & 35.20 & 115.8 & 11.42 & 1.70 \\
\hline Lefas and Kotsovos [105] & 0 & 65 & 140 & 3.300 & 0.900 & 470.0 & 1300 & 650 & 65 & 1.500 & 0.350 & 470.0 & 53.60 & 111.0 & 8 & 1.88 \\
\hline Lefas and Kotsovos [105] & 0 & 65 & 140 & 3.300 & 0.900 & 470.0 & 1300 & 650 & 65 & 1.500 & 0.350 & 470.0 & 49.20 & 111.5 & 10 & 1.92 \\
\hline Pilakoutas and Elnashai [106] & 0 & 110 & 60 & 2.830 & 0.780 & 500.0 & 1200 & 600 & 60 & 0.310 & 0.390 & 550.0 & 36.90 & 104.0 & 19.3 & 2.00 \\
\hline Pilakoutas and Elnashai [106] & 0 & 60 & 60 & 3.020 & 0.170 & 540.0 & 1200 & 600 & 60 & 0.470 & 0.310 & 550.0 & 31.80 & 117.3 & 21.7 & 0.80 \\
\hline Pilakoutas and Elnashai [106] & 0 & 110 & 60 & 2.830 & 0.170 & 500.0 & 1200 & 600 & 60 & 0.310 & 0.310 & 550.0 & 38.60 & 107.8 & 20 & 1.83 \\
\hline Pilakoutas and Elnashai [106] & 0 & 60 & 60 & 3.020 & 0.780 & 540.0 & 1200 & 600 & 60 & 0.470 & 0.390 & 550.0 & 32.00 & 127.3 & 21.3 & 1.83 \\
\hline Pilakoutas and Elnashai [106] & 0 & 110 & 60 & 2.930 & 0.410 & 540.0 & 1200 & 600 & 60 & 0.310 & 0.310 & 550.0 & 45.80 & 95.3 & 18.6 & 2.16 \\
\hline Pilakoutas and Elnashai [106] & 0 & 110 & 60 & 2.930 & 0.450 & 540.0 & 1200 & 600 & 60 & 0.310 & 0.310 & 550.0 & 38.90 & 97.4 & 18.7 & 2.16 \\
\hline Gupta and Rangan [107] & 0 & 375 & 100 & 1.250 & 0.150 & 545.0 & 1000 & 1000 & 75 & 1.000 & 0.500 & 545.0 & 79.30 & 427.8 & N.A. & N.A. \\
\hline Gupta and Rangan [107] & 610 & 375 & 100 & 1.730 & 0.150 & 545.0 & 1000 & 1000 & 75 & 1.000 & 0.500 & 545.0 & 65.10 & 719.6 & N.A. & N.A. \\
\hline Gupta and Rangan [107] & 1230 & 375 & 100 & 2.140 & 0.150 & 545.0 & 1000 & 1000 & 75 & 1.000 & 0.500 & 545.0 & 69.00 & 850.7 & N.A. & N.A. \\
\hline Gupta and Rangan [107] & 0 & 375 & 100 & 1.840 & 0.150 & 533.2 & 1000 & 1000 & 75 & 1.500 & 0.500 & 533.2 & 75.20 & 600.0 & N.A. & N.A. \\
\hline Gupta and Rangan [107] & 610 & 375 & 100 & 2.260 & 0.150 & 533.2 & 1000 & 1000 & 75 & 1.500 & 0.500 & 533.2 & 73.10 & 790.2 & N.A. & N.A. \\
\hline Gupta and Rangan [107] & 1230 & 375 & 100 & 2.490 & 0.150 & 533.2 & 1000 & 1000 & 75 & 1.500 & 0.500 & 533.2 & 70.50 & 970.0 & N.A. & N.A. \\
\hline
\end{tabular}


Table A2. Cont.

\begin{tabular}{|c|c|c|c|c|c|c|c|c|c|c|c|c|c|c|c|c|}
\hline Reference & $\begin{array}{l}\text { A.L } \\
\text { kN }\end{array}$ & $\begin{array}{c}\text { ac } \\
\text { mm }\end{array}$ & $\begin{array}{c}\mathrm{bc} \\
\mathrm{mm}\end{array}$ & $\begin{array}{c}\rho_{v c} \\
\%\end{array}$ & $\begin{array}{c}\rho_{h c} \\
\%\end{array}$ & $\begin{array}{l}\text { Fyc } \\
\text { MPa }\end{array}$ & $\begin{array}{l}\text { hw } \\
\text { mm }\end{array}$ & $\begin{array}{l}\text { lw } \\
\mathrm{mm}\end{array}$ & $\begin{array}{l}\text { tw } \\
\text { mm }\end{array}$ & $\begin{array}{c}\rho_{v w} \\
\%\end{array}$ & $\begin{array}{c}\rho_{h w} \\
\%\end{array}$ & $\begin{array}{l}\text { Fyw } \\
\text { MPa }\end{array}$ & $\begin{array}{l}\text { Fcw } \\
\text { MPa }\end{array}$ & $\begin{array}{c}\mathbf{P} \\
\mathbf{k N}\end{array}$ & $\begin{array}{c}\mathrm{K} \\
\mathrm{kN} / \mathrm{mm}\end{array}$ & $\begin{array}{l}\mathrm{D} \\
\%\end{array}$ \\
\hline Gupta and Rangan [107] & 610 & 375 & 100 & 1.730 & 0.150 & 545.0 & 1000 & 1000 & 75 & 1.000 & 1.000 & 545.0 & 71.20 & 800.0 & N.A. & N.A \\
\hline Gupta and Rangan [107] & 310 & 375 & 100 & 0.630 & 0.150 & 545.0 & 1000 & 1000 & 75 & 1.000 & 0.500 & 545.0 & 60.50 & 486.6 & N.A. & N.A \\
\hline Mickleborough et al. [108] & 357 & 0 & 0 & 0.000 & 0.000 & 0.0 & 1500 & 750 & 125 & 1.170 & 0.390 & 460.0 & 44.70 & 156.0 & 70.52 & 0.43 \\
\hline Mickleborough et al. [108] & 493 & 0 & 0 & 0.000 & 0.000 & 0.0 & 1500 & 750 & 125 & 1.170 & 0.390 & 460.0 & 56.00 & 235.0 & 56.55 & 0.66 \\
\hline Mickleborough et al. [108] & 334 & 0 & 0 & 0.000 & 0.000 & 0.0 & 1125 & 750 & 125 & 1.170 & 0.390 & 460.0 & 29.50 & 217.0 & & 0.45 \\
\hline Mickleborough et al. [108] & 486 & 0 & 0 & 0.000 & 0.000 & 0.0 & 1125 & 750 & 125 & 1.170 & 0.390 & 460.0 & 57.30 & 289.0 & 173.61 & 0.43 \\
\hline Mickleborough et al. [108] & 360 & 0 & 0 & 0.000 & 0.000 & 0.0 & 750 & 750 & 125 & 1.170 & 0.390 & 460.0 & 57.30 & 420.0 & 269.2 & 0.54 \\
\hline Mickleborough et al. [108] & 477 & 0 & 0 & 0.000 & 0.000 & 0.0 & 750 & 750 & 125 & 1.170 & 0.390 & 460.0 & 29.50 & 400.0 & 284 & 0.93 \\
\hline Sittipunt et al. [109] & 0 & 250 & 250 & 2.290 & 0.540 & 473.0 & 1900 & 1000 & 100 & 0.390 & 0.520 & 450.0 & 36.60 & 491.0 & 137.8 & 2.90 \\
\hline Sittipunt et al. [109] & 0 & 250 & 250 & 2.290 & 0.540 & 473.0 & 1900 & 1000 & 100 & 0.520 & 0.790 & 450.0 & 35.80 & 608.0 & 185 & 2.80 \\
\hline Zhang and Wang [110] & 500 & 100 & 100 & 0.009 & 0.010 & 405.0 & 1500 & 700 & 100 & 0.007 & 0.010 & 405.0 & 36.80 & 201.2 & 49.79 & 2.08 \\
\hline Zhang and Wang [110] & 784 & 100 & 100 & 0.007 & 0.010 & 432.0 & 1500 & 700 & 100 & 0.0 & & 432.0 & 40.20 & 224.0 & 54 & 1.59 \\
\hline Zhang and Wang [110] & 595 & 100 & 100 & 0.018 & 0.010 & 375.0 & 1500 & 700 & 100 & 0.0 & 0.0 & 375.0 & 43.10 & 303.5 & 52.14 & 2.10 \\
\hline Adebar et al. [111] & 1500 & 380 & 203 & 0.670 & & 455.0 & 11760 & 1625 & 127 & 0.270 & & 455.0 & 49.00 & 160.0 & 42 & 2.39 \\
\hline Terzio & 0 & 120 & 250 & 5.150 & 0.680 & 440.0 & 750 & 1500 & 120 & 0.680 & 0.680 & 481.0 & 19.30 & 799.0 & 338 & 1.20 \\
\hline Terzi & 0 & 120 & 250 & 5.150 & 0.680 & 440.0 & 750 & 1500 & 120 & 0.680 & 0.680 & 481.0 & 25.80 & 666.0 & 282 & 1.20 \\
\hline Terzi & 0 & 120 & 250 & 5.150 & 0.680 & 473.0 & 750 & 1500 & 120 & 0.680 & 0.680 & 584.0 & 29.00 & 813.0 & 506.6 & 1.20 \\
\hline lu et al. [112] & 0 & 120 & 250 & 5.150 & 0.680 & 584.0 & 750 & 1500 & 120 & 0.680 & 0.680 & 584.0 & 32.10 & 383.0 & 340 & 1.20 \\
\hline lu et al. [112] & 0 & 120 & 250 & 5.150 & 0.680 & 519.0 & 500 & 1500 & 120 & 0.680 & 0.680 & 584.0 & 34.80 & 874.0 & 1440 & 1.40 \\
\hline Terzioglu et al. [112] & 0 & 120 & 250 & 5.150 & 0.340 & 528.0 & 1500 & 1500 & 120 & 0.680 & 0.340 & 584.0 & 35.00 & 710.0 & 226 & 1.60 \\
\hline Terzioglu et al. [112] & 0 & 120 & 250 & 5.150 & 0.680 & 528.0 & 1500 & 1500 & 120 & 0.680 & 0.680 & 584.0 & 22.60 & 735.0 & 186 & 1.40 \\
\hline Terzioglu et al. [112] & 0 & 120 & 90 & 5.150 & 0.340 & 473.0 & 750 & 1500 & 120 & 0.340 & 0.340 & 584.0 & 24.00 & 563.0 & 426.6 & 1.20 \\
\hline Terzioglu et al. [112] & 240 & 120 & 90 & 5.150 & 0.340 & 473.0 & 750 & 1500 & 120 & 0.340 & 0.340 & 584.0 & 26.30 & 789.0 & 800 & 1.20 \\
\hline Terzioglu et al. [112] & 480 & 120 & 90 & 5.150 & 0.340 & 473.0 & 750 & 1500 & 120 & 0.340 & 0.340 & 584.0 & 27.00 & 793.0 & 733.33 & 1.20 \\
\hline Terzioglu et al. [112] & 0 & 120 & 90 & 5.150 & 0.340 & 440.0 & 750 & 1500 & 120 & 0.340 & 0.340 & 481.0 & 23.70 & 635.0 & 480 & 1.20 \\
\hline Abdulridha and Palermo [113] & 0 & 160 & 150 & 1.300 & 1.700 & 410.0 & 2200 & 1000 & 150 & 0.880 & 0.880 & 425.0 & 30.50 & 156.0 & 12.34 & 3.20 \\
\hline Qazi et al. [114] & 110 & 0 & 0 & 0.300 & 0.200 & 500.0 & 610 & 900 & 80 & 0.300 & 0.200 & 500.0 & 35.00 & 138.0 & 37.93 & 1.21 \\
\hline Christidis and Trezos [115] & 0 & 0 & 0 & 14.330 & 6.690 & 588.0 & 1400 & 750 & 125 & 14.330 & 6.690 & 588.0 & 31.12 & 203.0 & 16.8 & 3.70 \\
\hline Christidis and Trezos [115] & 0 & 0 & 0 & 12.060 & 2.010 & & 1400 & 750 & 125 & 12.060 & & 580.0 & 31.12 & 177.0 & 11.69 & 3.60 \\
\hline Christidis and Trezos [115] & 0 & 0 & 0 & & & & & 750 & 125 & & & 580.0 & & 173.3 & & 3.60 \\
\hline Christidis and Trezos [115] & 0 & 0 & 0 & & & & & 750 & & & & 580.0 & & 157.6 & & 2.14 \\
\hline Wang et al. [116] & 1800 & 0 & 0 & 0.310 & 0.310 & 347.8 & 2000 & 1000 & 125 & 0.310 & 0.310 & 347.8 & 28.76 & 233.0 & 31.38 & 0.50 \\
\hline Wang et al. [116] & 1438 & 0 & 0 & 0.310 & 0.310 & 347.8 & 2000 & 1000 & 125 & 0.310 & 0.310 & 347.8 & 28.76 & 212.0 & 32.54 & 0.70 \\
\hline Wang et al. [116] & 2978 & 0 & 0 & 0.190 & 0.190 & 347.8 & 2000 & 1000 & 200 & 0.190 & 0.190 & 347.8 & 49.64 & 371.0 & 49.16 & 1.25 \\
\hline
\end{tabular}


Table A2. Cont.

\begin{tabular}{|c|c|c|c|c|c|c|c|c|c|c|c|c|c|c|c|c|}
\hline Reference & $\begin{array}{l}\text { A.L } \\
\text { kN }\end{array}$ & $\begin{array}{c}\text { ac } \\
\mathrm{mm}\end{array}$ & $\begin{array}{c}\text { bc } \\
\text { mm }\end{array}$ & $\begin{array}{c}\rho_{v c} \\
\%\end{array}$ & $\begin{array}{c}\rho_{h c} \\
\%\end{array}$ & $\begin{array}{c}\text { Fyc } \\
\text { MPa }\end{array}$ & $\begin{array}{l}\text { hw } \\
\text { mm }\end{array}$ & $\begin{array}{l}\text { lw } \\
\mathrm{mm}\end{array}$ & $\begin{array}{c}\text { tw } \\
\mathrm{mm}\end{array}$ & $\begin{array}{c}\rho_{v w} \\
\%\end{array}$ & $\begin{array}{c}\rho_{h w} \\
\%\end{array}$ & $\begin{array}{l}\text { Fyw } \\
\text { MPa }\end{array}$ & $\begin{array}{l}\text { Fcw } \\
\text { MPa }\end{array}$ & $\begin{array}{c}\mathbf{P} \\
\mathbf{k N}\end{array}$ & $\begin{array}{c}\mathrm{K} \\
\mathrm{kN} / \mathrm{mm} \\
\end{array}$ & $\begin{array}{l}\mathrm{D} \\
\%\end{array}$ \\
\hline Ren et al. [117] & 1400 & 180 & 180 & 3.140 & 0.310 & 432.0 & 1700 & 925 & 180 & 0.220 & 0.620 & 312.0 & 44.30 & 549.0 & 83.7 & 2.59 \\
\hline Hariri-Ardebili and Saouma [19] & 800 & 200 & 120 & 2.100 & 0.670 & 430.0 & 750 & 1300 & 100 & 0.800 & 0.770 & 430.0 & 79.00 & 1180.0 & & 0.94 \\
\hline Le Nguyen et al. [118] & 110 & 0 & 0 & 0.160 & 0.130 & 500.0 & 610 & 900 & 80 & 0.160 & & 500.0 & & 158.0 & & 0.98 \\
\hline Le Nguyen et al. [118] & 90 & 0 & 0 & & & 500.0 & 1500 & 600 & 80 & & & & & & & 0.86 \\
\hline Choi [119] & 300 & 200 & 200 & & & 400 & 950 & 1500 & 100 & & & & & 610.0 & & 1.70 \\
\hline Choi [] & 300 & 200 & 200 & 1.420 & 1.300 & 400.0 & 950 & 1500 & 100 & 0.870 & 0.480 & 400.0 & 41.91 & 695.0 & 109.6 & 1.89 \\
\hline Choi [119] & 300 & 200 & 200 & 1.420 & 1.300 & 400.0 & 950 & 1500 & 100 & 0.460 & 1.850 & 400.0 & 40.34 & 620.0 & 117.1 & 1.57 \\
\hline Choi $[119]$ & 300 & 200 & 200 & 1.420 & 1.300 & 400.0 & 950 & 1500 & 100 & 0.460 & 1.850 & 400.0 & 40.34 & 650.0 & 91.62 & 1.78 \\
\hline Cortés-Puentes and Palermo [120] & 0 & 0 & 0 & 0.180 & 0.210 & 495.0 & 2000 & 2000 & 100 & 0.180 & 0.210 & 495.0 & 22.50 & 145.0 & 24.2 & 1.85 \\
\hline Cortés-Puentes and Palermo [121] & 0 & 0 & 0 & 0.180 & 0.210 & 495.0 & 2000 & 2000 & 100 & 0.180 & 0.210 & 495.0 & 9.50 & 117.0 & 25.7 & 2.00 \\
\hline Cortés-Puentes and Palermo [122] & 0 & 225 & 225 & 1.000 & 0.510 & 495.0 & 2000 & 1550 & 125 & 0.180 & 0.200 & 495.0 & 20.00 & 243.0 & 105.3 & 1.50 \\
\hline Qiao et al. [123] & 100 & 160 & 160 & 0.785 & 0.220 & 338.2 & 850 & 1000 & 160 & 0.250 & 0.120 & 535.8 & 26.48 & 338.3 & 794 & 2.93 \\
\hline Blandon et al. [124] & 470 & 350 & 100 & 1.000 & 0.140 & 419.0 & 2400 & 2500 & 100 & 0.270 & 0.270 & 419.0 & 39.10 & 323.7 & & 0.80 \\
\hline$n$ et al. [124] & 470 & 350 & 100 & 1.000 & 0.140 & 419.0 & 2400 & 2500 & 100 & 0.260 & 0.260 & 419.0 & 40.10 & 320.0 & 61.73 & 1.20 \\
\hline Blandon et al. [124] & 470 & 350 & 100 & 1.780 & 0.140 & 419.0 & 2400 & 2500 & 100 & 0.270 & 0.270 & 419.0 & 39.20 & 324.3 & 130 & 0.92 \\
\hline Christidis et al. [125] & 0 & 0 & 0 & 1.200 & 0.110 & 580.0 & 1400 & 750 & 125 & 1.200 & 0.110 & 580.0 & 25.37 & 157.6 & 14.08 & 2.20 \\
\hline Liao et al. [126] & 300 & 170 & 170 & 2.780 & 0.590 & 387.0 & 820 & 860 & 85 & 0.550 & 0.550 & 397.0 & 49.20 & 636.0 & 1630 & 2.00 \\
\hline Liao et al. [126] & 300 & 170 & 170 & 2.780 & 0.590 & 387.0 & 820 & 1320 & 85 & 0.550 & 0.550 & 397.0 & 49.20 & 852.0 & 794 & 1.28 \\
\hline Liao et al. [126] & 600 & 170 & 170 & 2.780 & 0.590 & 387.0 & 820 & 1320 & 85 & 0.550 & 0.550 & 397.0 & 49.20 & 966.0 & 564.6 & 1.12 \\
\hline Dazio et al. [127] & 689 & 150 & 150 & 1.570 & 1.430 & 547.0 & 4030 & 2000 & 150 & 0.300 & 0.250 & 583.0 & 45.00 & 336.0 & 47.3 & 1.04 \\
\hline & & 150 & 150 & & & 583.0 & 4030 & 2000 & & & & 484.0 & & 359.0 & & 1.38 \\
\hline & & 150 & 230 & & & 601.0 & & 2000 & & & & 569.0 & & & & 2.03 \\
\hline Dazi & 695 & 0 & 0 & & & 576.0 & 4030 & 2000 & 150 & & & 583.0 & & 443 & 9 & 1.35 \\
\hline Dazio & 1474 & 150 & 230 & 0.670 & 0.390 & 576.0 & 4030 & 2000 & 150 & 0.270 & 0.250 & 583.0 & 38.30 & 439.0 & 64.4 & 1.36 \\
\hline Dazio et al. [127] & 1476 & 150 & 355 & 1.540 & 0.820 & 576.0 & 3990 & 2000 & 150 & 0.540 & 0.250 & 583.0 & 45.60 & 597.0 & 87.9 & 2.07 \\
\hline Zhi et al. [128] & 600 & 200 & 385 & 1.200 & 0.780 & 510.0 & 3450 & 1600 & 200 & 0.860 & 0.390 & 505.0 & 41.20 & 482.0 & 22.4 & 2.23 \\
\hline Zhi et al. [128] & 600 & 200 & 407 & 1.130 & 1.200 & 510.0 & 3450 & 1600 & 200 & 0.860 & 0.390 & 505.0 & 42.10 & 448.0 & 22.9 & 2.05 \\
\hline Zhi et al. [128] & 600 & 200 & 407 & 1.130 & 1.200 & 510.0 & 3450 & 1600 & 200 & 0.860 & 0.390 & 505.0 & 42.10 & 462.0 & 26.8 & 2.05 \\
\hline Zhi et al. [128] & 600 & 200 & 407 & 1.130 & 1.600 & 510.0 & 3450 & 1600 & 200 & 0.860 & 0.390 & 505.0 & 42.10 & 439.0 & 26.8 & 1.80 \\
\hline Zhi et al. [128] & 600 & 200 & 407 & 1.130 & 1.600 & 510.0 & 3450 & 1600 & 200 & 0.860 & 0.390 & 505.0 & 42.10 & 456.0 & 21.9 & 2.26 \\
\hline al. [128] & 600 & 200 & 407 & 1.130 & 1.600 & 510.0 & 3450 & 1600 & 200 & 0.860 & 0.390 & 505.0 & 42.10 & 462.0 & 21.3 & 1.80 \\
\hline 1. [128] & 750 & 200 & 415 & 1.940 & 1.200 & 510.0 & 3650 & 1700 & 200 & 1.150 & 0.390 & 505.0 & 41.20 & 580.0 & 22.4 & 2.55 \\
\hline Zhi et al. [128] & 750 & 200 & 415 & 1.940 & 1.200 & 510.0 & 3650 & 1700 & 200 & 1.150 & 0.390 & 505.0 & 42.10 & 605.0 & 23.4 & 2.62 \\
\hline Zhi et al. [128] & 750 & 200 & 415 & 1.940 & 1.200 & 510.0 & 3650 & 1700 & 200 & 1.150 & 0.390 & 505.0 & 42.10 & 598.0 & 17.2 & 3.03 \\
\hline
\end{tabular}


Table A2. Cont.

\begin{tabular}{|c|c|c|c|c|c|c|c|c|c|c|c|c|c|c|c|c|}
\hline Reference & $\begin{array}{l}\text { A.L } \\
\text { kN }\end{array}$ & $\begin{array}{c}\text { ac } \\
\mathrm{mm}\end{array}$ & $\begin{array}{l}\mathrm{bc} \\
\mathrm{mm}\end{array}$ & $\begin{array}{c}\rho_{v c} \\
\%\end{array}$ & $\begin{array}{c}\rho_{h c} \\
\%\end{array}$ & $\begin{array}{l}\text { Fyc } \\
\text { MPa }\end{array}$ & $\begin{array}{l}\mathrm{hw} \\
\mathrm{mm}\end{array}$ & $\begin{array}{l}1 \mathrm{w} \\
\mathrm{mm}\end{array}$ & $\begin{array}{l}\text { tw } \\
\text { mm }\end{array}$ & $\begin{array}{c}\rho_{v w} \\
\%\end{array}$ & $\begin{array}{c}\rho_{h w} \\
\%\end{array}$ & $\begin{array}{l}\text { Fyw } \\
\text { MPa }\end{array}$ & $\begin{array}{l}\text { Fcw } \\
\text { MPa }\end{array}$ & $\begin{array}{c}\mathrm{P} \\
\mathbf{k N}\end{array}$ & $\begin{array}{c}\mathrm{K} \\
\mathrm{kN} / \mathrm{mm}\end{array}$ & $\begin{array}{l}\mathrm{D} \\
\%\end{array}$ \\
\hline Shen et al. [129] & 325 & 120 & 225 & 1.740 & 0.550 & 335.0 & 1600 & 1000 & 120 & 0.600 & 0.550 & 235.0 & 31.00 & 272.7 & 14.75 & 2.50 \\
\hline Zhang et al. [130] & 74.85 & 140 & 140 & 0.760 & 0.250 & 410.0 & 1050 & 1200 & 140 & 0.250 & 0.250 & 384.0 & 55.56 & 400.0 & N.A. & 2.20 \\
\hline Zhang et al. [130] & 134.73 & 140 & 140 & 0.760 & 0.120 & 425.0 & 850 & 1000 & 140 & 0.250 & 0.250 & 290.0 & 22.76 & N.A. & N.A. & N.A. \\
\hline Zhang et al. [130] & 67.36 & 140 & 140 & 0.760 & 0.120 & 302.0 & 850 & 1000 & 140 & 0.250 & 0.250 & 290.0 & 45.30 & N.A. & N.A. & N.A. \\
\hline Zhang et al. [130] & 112.27 & 140 & 140 & 1.000 & 0.180 & 302.0 & 850 & 1000 & 140 & 0.250 & 0.125 & 397.0 & 24.65 & N.A. & N.A. & N.A. \\
\hline Zhang et al. [130] & 112.27 & 140 & 140 & 1.000 & 0.180 & 302.0 & 850 & 1000 & 140 & 0.250 & 0.250 & 397.0 & 24.65 & N.A. & N.A. & N.A. \\
\hline Zhang et al. [130] & 112.27 & 140 & 140 & 1.00 & 0.180 & 302 & 850 & 1000 & 140 & & & 397.0 & 24.65 & & N.A. & N.A. \\
\hline Zhang et al. [130] & 112.27 & 140 & 140 & 1.000 & 0.000 & 302.0 & 850 & 1000 & 140 & 0.150 & 0.150 & 397.0 & 24.65 & N.A. & N.A. & N.A. \\
\hline Zhang et al. [130] & 112.27 & 140 & 140 & 1.150 & 0.000 & 302.0 & 850 & 1000 & 140 & 0.150 & 0.150 & 397.0 & 24.65 & N.A. & N.A. & N.A. \\
\hline Zhang et al. [130] & 112.27 & 140 & 140 & 1.410 & 0.180 & 302.0 & 850 & 1000 & 140 & 0.150 & 0.150 & 397.0 & 24.65 & N.A. & N.A. & N.A. \\
\hline Hidalgo et al. [2] & 0 & N.A. & N.A. & N.A. & N.A. & N.A. & 2000 & 1000 & 120 & 0.251 & 0.131 & 392.0 & 19.40 & 198.0 & 130 & 0.66 \\
\hline Hidalgo et al. [2] & 0 & N.A. & N.A. & N.A. & N.A. & N.A. & 2000 & 1000 & 120 & 0.251 & 0.246 & 402.0 & 19.60 & 270.0 & 116.2 & 0.75 \\
\hline Hidalgo et al. [2] & 0 & N.A. & N.A. & N.A. & N.A. & N.A. & 2000 & 1000 & 120 & 0.251 & 0.381 & 402.0 & 19.50 & 324.0 & 79.9 & 0.75 \\
\hline Hidalgo et al. [2] & 0 & N.A. & N.A. & N.A. & N.A. & N.A. & 1800 & 1300 & 120 & 0.259 & 0.131 & 314.0 & 17.60 & 309.0 & 240 & 0.44 \\
\hline Hidalgo et al. [2] & 0 & N.A. & N.A. & N.A. & N.A. & N.A. & 1800 & 1300 & 120 & 0.125 & 0.246 & 471.0 & 18.10 & 364.0 & 137 & 0.63 \\
\hline Hidalgo et al. [2] & 0 & N.A. & N.A. & N.A. & N.A. & N.A. & 1800 & 1300 & 120 & 0.259 & 0.246 & 471.0 & 15.70 & 374.0 & 212.7 & 0.55 \\
\hline Hidalgo et al. [2] & 0 & N.A. & N.A. & N.A. & N.A. & N.A. & 1800 & 1300 & 100 & 0.255 & 0.255 & 366.0 & 17.60 & 258.0 & 59 & 0.54 \\
\hline Hidalgo et al. [2] & 0 & N.A. & N.A. & N.A. & N.A. & N.A. & 1800 & 1300 & 80 & 0.250 & 0.250 & 367.0 & 16.40 & 187.0 & 64.44 & 0.46 \\
\hline Hidalgo et al. [2] & 0 & N.A. & N.A. & N.A. & N.A. & N.A. & 1400 & 1400 & 100 & 0.255 & 0.127 & 362.0 & 16.30 & 235.0 & 218.57 & 0.35 \\
\hline Hidalgo et al. [2] & 0 & N.A. & N.A. & N.A. & N.A. & N.A. & 1400 & 1400 & 100 & 0.127 & 0.255 & 366.0 & 17.00 & 304.0 & 123.21 & 0.50 \\
\hline Hidalgo et al. [2] & 0 & N.A. & N.A. & N.A. & N.A. & N.A. & 1400 & 1400 & 100 & 0.255 & 0.255 & 370.0 & 18.10 & 289.0 & 205.71 & 0.35 \\
\hline Hidalgo et al. [2] & 0 & N.A. & N.A. & N.A. & N.A. & N.A. & 1200 & 1700 & 80 & 0.250 & 0.125 & 366.0 & 17.10 & 255.0 & 239.58 & 0.25 \\
\hline Hidalgo et al. [2] & 0 & N.A. & N.A. & N.A. & N.A. & N.A. & 1200 & 1700 & 80 & 0.125 & & 366.0 & 19.00 & 368.0 & 245 & 0.42 \\
\hline & 0 & & & & & & 1200 & 1700 & 80 & & & 366.0 & 18.80 & & & 0.37 \\
\hline Hidalgo et & 0 & & & N.A. & N.A. & N.A. & 1800 & 1300 & 100 & & & 0.0 & 24.20 & & & 0.28 \\
\hline Hidalgo et al. [2] & 0 & N.A. & N.A. & N.A. & N.A. & N.A. & 1800 & 1300 & 100 & 0.000 & 0.000 & 0.0 & 17.20 & 222.0 & 74.74 & 0.27 \\
\hline Hidalgo et al. [2] & 0 & N.A. & N.A. & N.A. & N.A. & N.A. & 1800 & 1300 & 100 & 0.000 & 0.250 & 431.0 & 24.20 & 333.0 & 75.81 & 0.36 \\
\hline Hidalgo et al. [2] & 0 & N.A. & N.A. & N.A. & N.A. & N.A. & 1800 & 1300 & 100 & 0.250 & 0.000 & 431.0 & 23.90 & 323.0 & 80.09 & 0.21 \\
\hline Hidalgo et al. [2] & 0 & N.A. & N.A. & N.A. & N.A. & N.A. & 1400 & 1400 & 100 & 0.000 & 0.000 & 0.0 & 23.90 & 352.0 & 105.35 & 0.60 \\
\hline Hidalgo et al. [2] & 0 & N.A. & N.A. & N.A. & N.A. & N.A. & 1400 & 1400 & 100 & 0.000 & 0.000 & 0.0 & 17.70 & 262.0 & 90 & 0.46 \\
\hline Hidalgo et al. [2] & 0 & N.A. & N.A. & N.A. & N.A. & N.A. & 1400 & 1400 & 100 & 0.000 & 0.250 & 431.0 & 23.90 & 491.0 & 87.14 & 0.64 \\
\hline Hidalgo et al. [2] & 0 & N.A. & N.A. & N.A. & N.A. & N.A. & 1400 & 1400 & 100 & 0.250 & 0.000 & 431.0 & 23.30 & 258.0 & 119.84 & 0.31 \\
\hline Hidalgo et al. [2] & 0 & N.A. & N.A. & N.A. & N.A. & N.A. & 1050 & 1500 & 80 & 0.000 & 0.000 & 0.0 & 23.20 & 400.0 & 216.19 & 0.51 \\
\hline Hidalgo et al. [2] & 0 & N.A. & N.A. & N.A. & N.A. & N.A. & 1050 & 1500 & 80 & 0.000 & 0.000 & 0.0 & 17.90 & 356.0 & 252.38 & 0.63 \\
\hline
\end{tabular}


Table A2. Cont.

\begin{tabular}{|c|c|c|c|c|c|c|c|c|c|c|c|c|c|c|c|c|}
\hline Reference & $\begin{array}{l}\text { A.L } \\
\text { kN }\end{array}$ & $\begin{array}{c}\text { ac } \\
\mathrm{mm}\end{array}$ & $\begin{array}{c}\mathrm{bc} \\
\mathrm{mm}\end{array}$ & $\begin{array}{c}\rho_{v c} \\
\%\end{array}$ & $\begin{array}{c}\rho_{h c} \\
\%\end{array}$ & $\begin{array}{l}\text { Fyc } \\
\text { MPa }\end{array}$ & $\begin{array}{l}\text { hw } \\
\text { mm }\end{array}$ & $\begin{array}{l}\text { lw } \\
\text { mm }\end{array}$ & $\begin{array}{l}\text { tw } \\
\mathrm{mm}\end{array}$ & $\begin{array}{c}\rho_{v w} \\
\%\end{array}$ & $\underset{\%}{\rho_{h w}}$ & $\begin{array}{l}\text { Fyw } \\
\text { MPa }\end{array}$ & $\begin{array}{l}\text { Fcw } \\
\text { MPa }\end{array}$ & $\begin{array}{c}\mathbf{P} \\
\mathbf{k N}\end{array}$ & $\begin{array}{c}\mathrm{K} \\
\mathrm{kN} / \mathrm{mm}\end{array}$ & $\begin{array}{l}\text { D } \\
\%\end{array}$ \\
\hline Hidalgo et al. [2] & 0 & N.A. & N.A. & N.A. & N.A. & N.A. & 1050 & 1500 & 80 & 0.000 & 0.250 & 431.0 & 23.10 & 391.0 & 211.1 & 0.35 \\
\hline Hidalgo et al. [2] & 0 & N.A. & N.A. & N.A. & N.A. & N.A. & 1050 & 1500 & 80 & 0.250 & 0.000 & 431.0 & 23.30 & 344.0 & 206.35 & 0.38 \\
\hline Ji et al. [131] & 617 & 180 & 280 & 5.600 & 1.500 & 349.0 & 1350 & 1500 & 180 & 0.580 & 0.370 & 396.3 & 62.90 & 960.2 & 86.3 & 1.90 \\
\hline Ji et al. [131] & 1030 & 180 & 280 & 5.600 & 1.500 & 349.0 & 1350 & 1500 & 180 & 0.580 & 0.370 & 396.3 & 63.40 & 822.8 & 76.13 & 1.70 \\
\hline Ji et al. [131] & 1716 & 180 & 280 & 5.600 & 1.500 & 349.0 & 1350 & 1500 & 180 & 0.580 & 0.370 & 396.3 & 63.60 & 567.5 & 81.83 & 1.80 \\
\hline Ji et al. [131] & 2553 & 180 & 280 & 5.600 & 1.500 & 478.3 & 1350 & 1500 & 180 & 0.580 & 0.370 & 465.0 & 56.70 & 460.0 & 93.14 & 3.40 \\
\hline Ji et al. [131] & 3192 & 180 & 280 & 5.600 & 1.500 & 478.3 & 1350 & 1500 & 180 & 0.580 & 0.370 & 465.0 & 58.10 & 302.0 & 23.2 & 2.30 \\
\hline Ji et al. [131] & 0 & 180 & 280 & 5.600 & 1.500 & 478.3 & 1350 & 1500 & 180 & 0.580 & 0.370 & 465.0 & 55.40 & 1276.0 & 109.8 & 1.50 \\
\hline Mohamed et al. [132] & 823.2 & 200 & 140 & 0.500 & 0.630 & 400.0 & 3550 & 1500 & 200 & 0.230 & 0.630 & 400.0 & 39.20 & 476.0 & 36.5 & 2.60 \\
\hline Mohamed et al. [132] & 838 & 200 & 140 & 1.430 & 0.890 & 1412.0 & 3550 & 1500 & 200 & 0.580 & 1.580 & 1412.0 & 39.90 & 586.0 & 26.3 & 3.10 \\
\hline Mohamed et al. [132] & 668.6 & 200 & 140 & 0.000 & 0.000 & 1412.0 & 3550 & 1200 & 200 & 0.620 & 0.000 & 1412.0 & 39.80 & 449.0 & 19 & 3.00 \\
\hline Mohamed et al. [132] & 562.8 & 200 & 140 & 0.000 & 0.000 & 1412.0 & 3550 & 1000 & 200 & 0.590 & 0.000 & 1412.0 & 40.20 & 289.0 & 13.7 & 3.35 \\
\hline Quiroz et al. [133] & 186 & 250 & 100 & 1.500 & 0.180 & 450.0 & 2400 & 2650 & 100 & 0.180 & 0.180 & 450.0 & 17.16 & 355.0 & 170.68 & 1.08 \\
\hline Quiroz et al. [133] & 186 & 250 & 100 & 1.500 & 0.180 & 450.0 & 2400 & 2650 & 100 & 0.180 & 0.180 & 450.0 & 17.16 & 379.0 & 170.79 & 0.48 \\
\hline Quiroz et al. [133] & 186 & 250 & 100 & 1.500 & 0.180 & 450.0 & 2400 & 2650 & 100 & 0.180 & 0.180 & 450.0 & 17.16 & 419.0 & 201.46 & 0.78 \\
\hline Quiroz et al. [133] & 186 & 250 & 100 & 1.500 & 0.257 & 450.0 & 2400 & 2650 & 100 & 0.257 & 0.257 & 450.0 & 17.16 & 436.0 & 216.07 & 0.71 \\
\hline Quiroz et al. [133] & 186 & 250 & 100 & 1.500 & 0.257 & 450.0 & 2400 & 2650 & 100 & 0.257 & 0.257 & 450.0 & 17.16 & 416.0 & 227.44 & 0.53 \\
\hline Quiroz et al. [133] & 186 & 250 & 100 & 1.500 & 0.257 & 450.0 & 2400 & 2650 & 100 & 0.257 & 0.257 & 450.0 & 17.16 & 436.0 & 215.26 & 1.41 \\
\hline Quiroz et al. [133] & 186 & 250 & 100 & 1.500 & 0.284 & 450.0 & 2400 & 2650 & 100 & 0.284 & 0.284 & 450.0 & 17.16 & 456.0 & 217.62 & 1.04 \\
\hline Salonikios et al. [134] & 0 & 100 & 240 & 1.700 & 1.700 & 500.0 & 1200 & 1200 & 100 & 0.565 & 0.565 & 500.0 & 22.20 & 262.0 & 131 & 0.83 \\
\hline Salonikios et al. [134] & 0 & 100 & 240 & 1.300 & 1.700 & 500.0 & 1200 & 1200 & 100 & 0.277 & 0.277 & 500.0 & 21.60 & 191.0 & 73.46 & 0.70 \\
\hline Salonikios et al. [134] & 200 & 100 & 240 & 1.300 & 1.700 & 500.0 & 1200 & 1200 & 100 & 0.277 & 0.277 & 500.0 & 23.90 & 268.0 & 92.4 & 1.80 \\
\hline Salonikios et al. [134] & 0 & 100 & 240 & 1.700 & 1.100 & 500.0 & 1800 & 1200 & 100 & 0.565 & 0.565 & 500.0 & 26.10 & 197.0 & 24.32 & 1.50 \\
\hline Salonikios et al. [134] & 0 & 100 & 240 & 1.300 & 1.100 & 500.0 & 1800 & 1200 & 100 & 0.277 & 0.277 & 500.0 & 26.20 & 124.0 & 15 & 1.40 \\
\hline Salonikios et al. [134] & 202 & 100 & 240 & 1.300 & 1.100 & 500.0 & 1800 & 1200 & 100 & 0.277 & 0.277 & 500.0 & 24.10 & 176.0 & 34 & 2.05 \\
\hline Salonikios et al. [134] & 0 & 100 & 240 & 1.300 & 1.700 & 500.0 & 1800 & 1200 & 100 & 0.565 & 0.565 & 500.0 & 27.50 & 202.0 & 16.6 & 1.50 \\
\hline Thomsen [135] & 341 & 171 & 102 & 3.250 & 1.160 & 414.0 & 3660 & 1220 & 102 & 0.280 & 0.110 & 414.0 & 27.40 & 148.0 & 10.37 & 2.20 \\
\hline Thomsen [135] & 238 & 171 & 102 & 3.250 & 1.132 & 414.0 & 3660 & 1220 & 102 & 0.280 & 0.170 & 414.0 & 27.40 & 158.0 & 10.45 & 2.40 \\
\hline Tran and Wallace [136] & 878 & 180 & 150 & 3.230 & 0.250 & 400.0 & 2440 & 1220 & 150 & 0.270 & 0.270 & 400.0 & 48.00 & 481.0 & 74 & 3.11 \\
\hline Tran and Wallace [136] & 878 & 180 & 150 & 7.110 & 0.250 & 448.0 & 2440 & 1220 & 150 & 0.610 & 0.610 & 448.0 & 48.00 & 742.0 & 80.47 & 3.00 \\
\hline Tran and Wallace [136] & 878 & 180 & 150 & 3.230 & 0.250 & 400.0 & 1830 & 1220 & 150 & 0.320 & 0.320 & 400.0 & 48.00 & 603.0 & 146 & 3.20 \\
\hline Tran and Wallace [136] & 1024 & 180 & 150 & 6.060 & 0.250 & 448.0 & 1830 & 1220 & 150 & 0.730 & 0.730 & 448.0 & 56.00 & 859.0 & 134 & 3.00 \\
\hline Tran and Wallace [136] & 256 & 180 & 150 & 6.060 & 0.250 & 400.0 & 1830 & 1220 & 150 & 0.610 & 0.610 & 400.0 & 56.00 & 670.0 & 109.2 & 3.00 \\
\hline
\end{tabular}


Table A2. Cont.

\begin{tabular}{|c|c|c|c|c|c|c|c|c|c|c|c|c|c|c|c|c|}
\hline Reference & $\begin{array}{l}\text { A.L } \\
\text { kN }\end{array}$ & $\begin{array}{c}\text { ac } \\
\mathrm{mm}\end{array}$ & $\begin{array}{c}\text { bc } \\
\mathrm{mm}\end{array}$ & $\begin{array}{c}\rho_{v c} \\
\%\end{array}$ & $\begin{array}{c}\rho_{h c} \\
\%\end{array}$ & $\begin{array}{c}\text { Fyc } \\
\text { MPa }\end{array}$ & $\begin{array}{l}\text { hw } \\
\text { mm }\end{array}$ & $\begin{array}{l}\text { lw } \\
\mathrm{mm}\end{array}$ & $\begin{array}{c}\text { tw } \\
\mathrm{mm}\end{array}$ & $\begin{array}{c}\rho_{v w} \\
\%\end{array}$ & $\begin{array}{c}\rho_{h w} \\
\%\end{array}$ & $\begin{array}{l}\text { Fyw } \\
\text { MPa }\end{array}$ & $\begin{array}{l}\text { Fcw } \\
\text { MPa }\end{array}$ & $\begin{array}{c}P \\
\mathbf{k N}\end{array}$ & $\begin{array}{c}\mathrm{K} \\
\mathrm{kN} / \mathrm{mm}\end{array}$ & $\begin{array}{l}\text { D } \\
\%\end{array}$ \\
\hline Layssi and Mitchell [137] & 0 & 0 & 0 & 0.830 & 0.260 & 460.0 & 3250 & 1200 & 150 & 0.830 & 0.260 & 470.0 & 31.20 & 95.2 & 39.33 & 0.47 \\
\hline Layssi and Mitchell [137] & 0 & 0 & 0 & 1.500 & 0.260 & 460.0 & 3250 & 1200 & 150 & 1.500 & 0.260 & 470.0 & 30.40 & 140.5 & 25.12 & 0.50 \\
\hline Ghorbani-Renani et al. [138] & 0 & 200 & 150 & 5.330 & 0.660 & 429.0 & 2700 & 1300 & 200 & 0.600 & 0.660 & 440.0 & 28.30 & 488.2 & 39 & 4.60 \\
\hline Ghorbani-Renani et al. [138] & 0 & 200 & 150 & 5.330 & 0.660 & 429.0 & 2700 & 1300 & 200 & 0.600 & 0.660 & 440.0 & 28.30 & 479.0 & 39 & 4.00 \\
\hline Ghorbani-Renani et al. [138] & 0 & 200 & 60 & 2.360 & 0.660 & 437.0 & 1140 & 548 & 84 & 0.480 & 0.660 & 437.0 & 47.00 & 86.7 & 19.95 & 3.23 \\
\hline Ghorbani-Renani et al. [138] & 0 & 200 & 60 & 2.360 & 0.660 & 437.0 & 1140 & 548 & 84 & 0.480 & 0.660 & 437.0 & 47.00 & 88.5 & 19.95 & 4.00 \\
\hline Ghazizadeh et al. [139] & 0 & 150 & 240 & 2.200 & 2.000 & 414.0 & 1800 & 1800 & 150 & 0.600 & 1.000 & 414.0 & 61.00 & 588.0 & 200 & 2.20 \\
\hline Ghazizadeh and Cruz-Noguez [140] & 0 & 150 & 240 & 1.050 & 0.840 & 1254.0 & 1800 & 1800 & 150 & 0.560 & 1.000 & 1254.0 & 40.00 & 550.0 & 207 & 2.20 \\
\hline Athanasopoulou [141] & 0 & 101 & 76.2 & 5.000 & 0.530 & 512.0 & 1016 & 1016 & 101 & 0.710 & 0.710 & 660.0 & 46.19 & 362.0 & 45.44 & 2.50 \\
\hline Athanasopoulou [141] & 0 & 101 & 101 & 9.400 & 1.000 & 453.0 & 1016 & 1016 & 101 & 0.830 & 0.830 & 444.0 & 46.19 & 476.0 & 92.64 & 2.30 \\
\hline Athanasopoulou [141] & 0 & 101 & 76.2 & 9.400 & 0.980 & 480.0 & 1320 & 1016 & 101 & 0.710 & 0.710 & 660.0 & 46.88 & 392.7 & 45.44 & 2.20 \\
\hline Athanasopoulou [141] & 0 & 101 & 101 & 13.000 & 1.400 & 482.0 & 1320 & 1016 & 101 & 0.670 & 0.670 & 463.0 & 42.74 & 529.0 & 125.8 & 2.20 \\
\hline Athanasopoulou [141] & 0 & 101 & 101 & 11.000 & 1.200 & 482.0 & 1320 & 1016 & 101 & 0.500 & 0.500 & 463.0 & 37.92 & 476.0 & 83.9 & 2.40 \\
\hline Tupper [142] & 600 & 152 & 140 & 11.000 & 0.240 & 450.0 & 3900 & 1000 & 152 & 0.280 & 0.490 & 450.0 & 38.70 & 334.0 & 8.97 & 2.90 \\
\hline Arafa et al. [143] & 0 & 200 & 200 & 1.430 & 0.890 & 1372.0 & 2000 & 1500 & 200 & 0.590 & 0.510 & 1372.0 & 35.00 & 678.0 & 294 & 2.55 \\
\hline Arafa et al. [143] & 0 & 200 & 200 & 1.430 & 0.890 & 1372.0 & 2000 & 1500 & 200 & 0.590 & 0.790 & 1372.0 & 35.00 & 708.0 & 290 & 2.80 \\
\hline Arafa et al. [143] & 0 & 200 & 200 & 1.430 & 0.890 & 1372.0 & 2000 & 1500 & 200 & 0.590 & 1.580 & 1372.0 & 40.00 & 912.0 & 315 & 3.00 \\
\hline Arafa et al. [143] & 0 & 200 & 200 & 1.430 & 0.890 & 1372.0 & 2000 & 1500 & 200 & 0.590 & 3.560 & 1372.0 & 41.00 & 935.0 & 323 & 3.10 \\
\hline Arafa et al. [143] & 0 & 200 & 200 & 1.430 & 0.890 & 1372.0 & 2000 & 1500 & 200 & 0.590 & 0.000 & 1372.0 & 38.00 & 398.0 & 306 & 2.20 \\
\hline Arafa et al. [143] & 0 & 200 & 200 & 1.430 & 0.890 & 1372.0 & 2000 & 1500 & 200 & 0.000 & 0.510 & 1372.0 & 35.00 & 482.0 & 231.6 & 2.40 \\
\hline G. Oesterle et al. [144] & 0 & 0 & 0 & 1.470 & 0.000 & 511.6 & 4570 & 1910 & 102 & 0.310 & 0.250 & 511.6 & 44.71 & 118.0 & 13.7 & 2.30 \\
\hline G. Oesterle et al. [144] & 0 & 0 & 0 & 4.000 & 2.070 & 450.2 & 4570 & 1910 & 102 & 0.310 & 0.250 & 450.2 & 46.37 & 216.0 & 15.87 & 2.90 \\
\hline G. Oesterle et al. [144] & 0 & 305 & 305 & 1.110 & 0.000 & 449.6 & 4570 & 1300 & 102 & 0.310 & 0.290 & 449.6 & 52.98 & 271.0 & 28.6 & 3.30 \\
\hline G. Oesterle et al. [144] & 0 & 305 & 305 & 3.670 & 0.000 & 410.3 & 4570 & 1300 & 102 & 0.630 & 0.290 & 410.3 & 53.60 & 680.0 & 33 & 2.30 \\
\hline G. Oesterle et al. [144] & 0 & 305 & 305 & 1.110 & 1.280 & 437.8 & 4570 & 1300 & 102 & 0.310 & 0.290 & 437.8 & 47.29 & 276.0 & 25.9 & 3.90 \\
\hline G. Oesterle et al. [144] & 0 & 305 & 305 & 1.110 & 1.280 & 450.2 & 4570 & 1300 & 102 & 0.310 & 0.290 & 450.2 & 45.02 & 335.0 & N.A. & 6.90 \\
\hline G. Oesterle et al. [144] & 0 & 305 & 305 & 3.670 & 1.350 & 444.0 & 4570 & 1300 & 102 & 0.630 & 0.290 & 444.0 & 45.30 & 762.0 & 34 & 2.70 \\
\hline Oesterle et al. [145] & 272.59 & 305 & 305 & 3.670 & 0.810 & 440.6 & 4570 & 1300 & 102 & 0.630 & 0.290 & 440.6 & 21.82 & 825.5 & N.A. & 2.80 \\
\hline Oesterle et al. [145] & 349.56 & 305 & 305 & 3.670 & 1.350 & 457.8 & 4570 & 1300 & 102 & 1.380 & 0.290 & 457.8 & 49.33 & 980.0 & 172 & 2.70 \\
\hline Oesterle et al. [145] & 349.56 & 305 & 305 & 3.670 & 1.350 & 447.5 & 4570 & 1300 & 102 & 0.630 & 0.290 & 447.5 & 41.97 & 978.0 & N.A. & 2.90 \\
\hline Oesterle et al. [145] & 349.56 & 305 & 305 & 3.670 & 1.350 & 429.6 & 4570 & 1300 & 102 & 0.630 & 0.290 & 429.6 & 44.09 & 977.0 & N.A. & 3.00 \\
\hline Oesterle et al. [145] & 349.56 & 305 & 305 & 1.670 & 1.350 & 447.5 & 4570 & 1300 & 102 & 0.630 & 0.290 & 447.5 & 45.61 & 707.0 & N.A. & 2.80 \\
\hline Shiu et al. [146] & 0 & 106 & 318 & 5.000 & 0.000 & 473.0 & 5500 & 1905 & 106 & 0.240 & 0.360 & 473.0 & 23.30 & 338.6 & 7.53 & 2.86 \\
\hline
\end{tabular}


Table A2. Cont.

\begin{tabular}{|c|c|c|c|c|c|c|c|c|c|c|c|c|c|c|c|c|}
\hline Reference & $\begin{array}{l}\text { A.L } \\
\text { kN }\end{array}$ & $\begin{array}{c}\text { ac } \\
\text { mm }\end{array}$ & $\begin{array}{l}\mathrm{bc} \\
\mathrm{mm}\end{array}$ & $\begin{array}{c}\rho_{v c} \\
\%\end{array}$ & $\begin{array}{c}\rho_{h c} \\
\%\end{array}$ & $\begin{array}{l}\text { Fyc } \\
\text { MPa }\end{array}$ & $\begin{array}{l}\text { hw } \\
\text { mm }\end{array}$ & $\begin{array}{l}1 \mathrm{w} \\
\mathrm{mm}\end{array}$ & $\begin{array}{l}\mathrm{tw} \\
\mathrm{mm}\end{array}$ & $\underset{\%}{\rho_{v w}}$ & $\begin{array}{c}\rho_{h w} \\
\%\end{array}$ & $\begin{array}{l}\text { Fyw } \\
\text { MPa }\end{array}$ & $\begin{array}{l}\text { Fcw } \\
\text { MPa }\end{array}$ & $\begin{array}{c}P \\
k N\end{array}$ & $\begin{array}{c}\mathrm{K} \\
\mathrm{kN} / \mathrm{mm}\end{array}$ & $\begin{array}{l}\mathrm{D} \\
\%\end{array}$ \\
\hline JIANG and LU [147] & 200 & 67 & 95 & 0.031 & 0.010 & 297.0 & 933 & 1667 & 67 & 0.010 & 0.010 & 325.0 & 18.30 & 521.8 & 375 & 0.66 \\
\hline JIANG and LU [147] & 400 & 67 & 95 & 0.031 & 0.010 & 297.0 & 933 & 1667 & 67 & 0.010 & 0.010 & 325.0 & 18.30 & 587.2 & 387 & 0.79 \\
\hline JIANG and LU [147] & 0 & 67 & 95 & 0.031 & 0.010 & 297.0 & 933 & 827 & 67 & 0.010 & 0.010 & 325.0 & 19.10 & 316.3 & 371.7 & 0.80 \\
\hline JIANG and LU [147] & 200 & 67 & 95 & 0.031 & 0.010 & 297.0 & 933 & 827 & 67 & 0.010 & 0.010 & 325.0 & 19.10 & 399.5 & 379.1 & 0.95 \\
\hline JIANG and LU [147] & 400 & 67 & 95 & 0.031 & 0.010 & 297.0 & 933 & 827 & 67 & 0.010 & 0.010 & 325.0 & 19.10 & 487.9 & 483 & 0.87 \\
\hline JIANG and LU [147] & 0 & 67 & 95 & 0.031 & 0.010 & 297.0 & 933 & 827 & 67 & 0.010 & 0.010 & 325.0 & 16.60 & 307.3 & 300 & 0.80 \\
\hline JIANG and LU [147] & 200 & 67 & 95 & 0.0 & 0.0 & 297.0 & 933 & 827 & 67 & & & 325.0 & 16.60 & 381 & 370 & 0.67 \\
\hline and LU [147] & 400 & 67 & 95 & 0.0 & 0.010 & 297.0 & 93 & 827 & 67 & 0.010 & 0.010 & 325.0 & 16.60 & 469.1 & 402.4 & 0.65 \\
\hline JIANG and LU [147] & 0 & 67 & 95 & 0.031 & 0.010 & 297.0 & 933 & 827 & 67 & 0.010 & 0.010 & 325.0 & 16.20 & 307.7 & 277.9 & 0.64 \\
\hline JIANG and LU [147] & 200 & 67 & 95 & 0.031 & 0.010 & 297.0 & 933 & 827 & 67 & 0.010 & 0.010 & 325.0 & 16.20 & 393.6 & 350.7 & 0.57 \\
\hline JIANG and LU [147] & 400 & 67 & 95 & 0.031 & 0.010 & 297.0 & 933 & 827 & 67 & 0.010 & 0.010 & 325.0 & 16.20 & 473.0 & 385.1 & 0.66 \\
\hline Park et al. [148] & 970 & 200 & 300 & 9.700 & 0.000 & 617.0 & 1500 & 1500 & 200 & 0.660 & 0.510 & 653.0 & 46.50 & 2158.0 & 171 & 1.00 \\
\hline Park et al. [148] & 970 & 200 & 300 & 9.700 & 0.000 & 617.0 & 1500 & 1500 & 200 & 0.660 & 0.680 & 653.0 & 46.50 & 2298.0 & 105 & 1.00 \\
\hline Park et al. [148] & 1470 & 200 & 300 & 9.700 & 0.000 & 617.0 & 1500 & 1500 & 200 & 0.660 & 0.510 & 653.0 & 70.30 & 2085.0 & 397 & 0.75 \\
\hline Park et al. [148] & 970 & 300 & 200 & 9.700 & 3.830 & 617.0 & 1500 & 1500 & 200 & 0.540 & 0.510 & 653.0 & 46.50 & 2544.0 & 542 & 1.00 \\
\hline Park et al. [148] & 970 & 200 & 200 & 9.700 & 0.000 & 617.0 & 1500 & 1500 & 200 & 0.360 & 0.250 & 653.0 & 46.10 & 1477.0 & 133 & 1.00 \\
\hline Park et al. [148] & 1470 & 200 & 200 & 9.700 & 0.000 & 617.0 & 1500 & 1500 & 200 & 0.360 & 0.250 & 653.0 & 70.30 & 1876.0 & 222 & 1.00 \\
\hline Park et al. [148] & 970 & 200 & 200 & 9.700 & 2.300 & 617.0 & 1500 & 1500 & 200 & 0.360 & 0.250 & 653.0 & 46.50 & 1915.0 & 328 & 0.90 \\
\hline Park et al. [148] & 970 & 200 & 120 & 2.000 & 2.300 & 653.0 & 1500 & 1500 & 200 & 0.360 & 0.250 & 653.0 & 46.50 & 1149.0 & 139 & 2.85 \\
\hline Hube et al. [149] & 0 & 0 & 0 & 0.800 & 1.250 & 500.0 & 1600 & 1600 & 100 & 0.200 & 0.200 & 500.0 & 28.90 & 349.0 & 135.5 & 0.39 \\
\hline Hube et al. [149] & 0 & 0 & 0 & 0.300 & 1.250 & 500.0 & 1600 & 1600 & 10 & 0.200 & 0.200 & 500.0 & 28.90 & 233.0 & 78.7 & 0.75 \\
\hline & 0 & 0 & 0 & & 1.2 & 500.0 & & 1600 & 10 & & 0.260 & 500.0 & 28.90 & 471.0 & 89 & 0.61 \\
\hline & 0 & 0 & 0 & & & 500.0 & & & & & & 500.0 & 28.90 & 403.0 & 98 & 0.55 \\
\hline & 0 & 0 & 0 & & & 500.0 & 16 & 1600 & & 0.200 & 0.2 & 500.0 & 28.90 & 357.0 & 113.8 & 0.80 \\
\hline & 0 & 0 & 0 & 0.800 & 1.250 & 500.0 & 1600 & 1600 & 100 & 0.140 & 0.140 & 500.0 & 28.90 & 416.0 & 58.5 & 0.93 \\
\hline Hube et al. [149] & 0 & 0 & 0 & 0.600 & 1.250 & 500.0 & 1600 & 1600 & 80 & 0.250 & 0.250 & 500.0 & 28.90 & 324.0 & 171 & 0.61 \\
\hline Hube et al. [149] & 0 & 0 & 0 & 0.600 & 1.250 & 500.0 & 1600 & 1600 & 80 & 0.170 & 0.170 & 500.0 & 28.90 & 292.0 & 43 & 0.65 \\
\hline Hube et al. [149] & 0 & 0 & 0 & 0.600 & 1.250 & 500.0 & 1600 & 1600 & 80 & 0.250 & 0.250 & 500.0 & 28.90 & 336.0 & 74 & 1.25 \\
\hline Alarcon et al. [150] & 287 & 100 & 140 & 0.450 & 0.440 & 469.0 & 1600 & 700 & 100 & 0.720 & 0.440 & 445.0 & 27.40 & 144.3 & 10.06 & 2.70 \\
\hline Alarcon et al. [150] & 479 & 100 & 140 & 0.450 & 0.440 & 469.0 & 1600 & 700 & 100 & 0.720 & 0.440 & 445.0 & 27.40 & 166.0 & 15.3 & 1.80 \\
\hline Alarcon et al. [150] & 671.6 & 100 & 140 & 0.450 & 0.440 & 469.0 & 1600 & 700 & 100 & 0.720 & 0.440 & 445.0 & 27.40 & 185.6 & 17.4 & 1.50 \\
\hline
\end{tabular}


Table A2. Cont.

\begin{tabular}{|c|c|c|c|c|c|c|c|c|c|c|c|c|c|c|c|c|}
\hline Reference & $\begin{array}{l}\text { A.L } \\
\text { kN }\end{array}$ & $\begin{array}{c}\text { ac } \\
\text { mm }\end{array}$ & $\begin{array}{c}\mathrm{bc} \\
\mathrm{mm}\end{array}$ & $\begin{array}{c}\rho_{v c} \\
\%\end{array}$ & $\begin{array}{c}\rho_{h c} \\
\%\end{array}$ & $\begin{array}{l}\text { Fyc } \\
\text { MPa }\end{array}$ & $\begin{array}{l}\text { hw } \\
\text { mm }\end{array}$ & $\begin{array}{l}\mathbf{l w} \\
\mathrm{mm}\end{array}$ & $\begin{array}{c}\mathrm{tw} \\
\mathrm{mm}\end{array}$ & $\begin{array}{c}\rho_{v w} \\
\%\end{array}$ & $\begin{array}{c}\rho_{h w} \\
\%\end{array}$ & $\begin{array}{l}\text { Fyw } \\
\text { MPa }\end{array}$ & $\begin{array}{l}\text { Fcw } \\
\text { MPa }\end{array}$ & $\begin{array}{c}P \\
\mathbf{k N}\end{array}$ & $\begin{array}{c}\mathrm{K} \\
\mathrm{kN} / \mathrm{mm}\end{array}$ & $\begin{array}{l}\text { D } \\
\%\end{array}$ \\
\hline Kabeyasawa and Matsumoto [151] & 1764 & 200 & 200 & 2.130 & 0.800 & 776.0 & 3000 & 1300 & 80 & 0.530 & 0.530 & 1001.0 & 87.60 & 1062.0 & 134.5833 & 1.90 \\
\hline Kabeyasawa and Matsumoto [151] & 1764 & 200 & 200 & 2.130 & 0.800 & 776.0 & 2000 & 1300 & 80 & 0.530 & 0.530 & 1001.0 & 93.60 & 1468.0 & 228.5714 & 1.49 \\
\hline Kabeyasawa and Matsumoto [151] & 1372 & 200 & 200 & 2.130 & 0.490 & 713.0 & 3000 & 1300 & 80 & 0.265 & 0.265 & 753.0 & 55.50 & 717.0 & 235.8025 & 0.99 \\
\hline Kabeyasawa and Matsumoto [151] & 1568 & 200 & 200 & 2.840 & 0.490 & 713.0 & 3000 & 1300 & 80 & 0.265 & 0.265 & 753.0 & 54.60 & 784.0 & 122.2222 & 0.93 \\
\hline Kabeyasawa and Matsumoto [151] & 1372 & 200 & 200 & 2.840 & 0.490 & 713.0 & 3000 & 1300 & 80 & 0.530 & 0.530 & 753.0 & 60.30 & 900.0 & 422.2222 & 1.52 \\
\hline Kabeyasawa and Matsumoto [151] & 1568 & 200 & 200 & 3.810 & 0.490 & 726.0 & 3000 & 1300 & 80 & 0.530 & 0.530 & 753.0 & 65.20 & 1056.0 & 160.6667 & 1.31 \\
\hline Luna et al. [152] & 0 & 0 & 0 & 0.000 & 0.000 & 462.3 & 2867 & 3050 & 203 & 0.670 & 0.670 & 462.3 & 24.84 & 1125.9 & 331.2 & 1.30 \\
\hline Luna et al. [152] & 0 & 0 & 0 & 0.000 & 0.000 & 434.7 & 1647 & 3050 & 203 & 1.000 & 1.000 & 434.7 & 48.30 & 2505.4 & 124 & 1.25 \\
\hline Luna et al. [152] & 0 & 0 & 0 & 0.000 & 0.000 & 434.7 & 1647 & 3050 & 203 & 0.670 & 0.670 & 434.7 & 53.82 & 2082.6 & 61.2 & 2.09 \\
\hline Luna et al. [152] & 0 & 0 & 0 & 0.000 & 0.000 & 462.3 & 1647 & 3050 & 203 & 0.330 & 0.330 & 462.3 & 28.98 & 1005.7 & 57.6 & 1.08 \\
\hline Luna et al. [152] & 0 & 0 & 0 & 0.000 & 0.000 & 462.3 & 1006 & 3050 & 203 & 1.000 & 1.000 & 462.3 & 29.67 & 3230.7 & 1602 & 0.89 \\
\hline Luna et al. [152] & 0 & 0 & 0 & 0.000 & 0.000 & 462.3 & 1006 & 3050 & 203 & 0.670 & 0.670 & 462.3 & 26.22 & 2541.0 & 1362.6 & 0.81 \\
\hline Luna et al. [152] & 0 & 0 & 0 & 0.000 & 0.000 & 462.3 & 1006 & 3050 & 203 & 0.330 & 0.330 & 462.3 & 26.22 & 1415.1 & 2206.8 & 0.45 \\
\hline Luna et al. [152] & 0 & 0 & 0 & 0.000 & 0.000 & 462.3 & 1647 & 3050 & 203 & 1.500 & 1.500 & 462.3 & 24.15 & 2772.4 & 966.6 & 0.70 \\
\hline Luna et al. [152] & 0 & 0 & 0 & 0.000 & 0.000 & 462.3 & 1647 & 3050 & 203 & 1.500 & 0.670 & 462.3 & 29.67 & 2767.9 & 896.4 & 0.60 \\
\hline Luna et al. [152] & 0 & 0 & 0 & 0.000 & 0.000 & 462.3 & 1647 & 3050 & 203 & 1.500 & 0.330 & 462.3 & 31.74 & 2202.8 & 945 & 0.52 \\
\hline Luna et al. [152] & 0 & 203 & 395 & 1.500 & 1.500 & 462.3 & 1647 & 3050 & 203 & 0.670 & 0.670 & 462.3 & 34.50 & 1886.8 & 736.2 & 0.56 \\
\hline Luna et al. [152] & 0 & 203 & 295 & 2.000 & 2.000 & 462.3 & 1647 & 3050 & 203 & 0.330 & 0.330 & 462.3 & 34.50 & 1624.3 & 693 & 0.89 \\
\hline Altin et al. [153] & 0 & 100 & 100 & 2.000 & 0.150 & 425.0 & 1500 & 1000 & 100 & 1.830 & 0.150 & 325.0 & 15.50 & 149.0 & 47.6 & 1.05 \\
\hline Aaleti et al. [154] & 0 & 150 & 200 & 5.000 & 0.850 & 413.0 & 6400 & 2300 & 150 & 0.370 & 0.490 & 413.7 & 35.40 & 840.0 & 28 & 2.00 \\
\hline Segura Jr and Wallace [155] & 1201 & 330 & 152.4 & 3.180 & 0.920 & 531.0 & 2134 & 2286 & 152.4 & 0.460 & 0.450 & 578.0 & 35.80 & 419.0 & 124.6 & 2.20 \\
\hline Segura Jr and Wallace [155] & 1201 & 330 & 152.4 & 3.180 & 1.300 & 531.0 & 2134 & 2286 & 152.4 & 0.460 & 0.450 & 578.0 & 41.70 & 440.0 & 127 & 2.10 \\
\hline Segura Jr and Wallace [155] & 1201 & 330 & 152.4 & 3.180 & 0.980 & 531.0 & 2134 & 2286 & 152.4 & 0.460 & 0.450 & 578.0 & 42.40 & 445.0 & 103.8 & 3.00 \\
\hline Segura Jr and Wallace [155] & 1503 & 330 & 191 & 3.140 & 1.600 & 531.0 & 2134 & 2286 & 191 & 0.460 & 0.490 & 578.0 & 46.30 & 583.0 & 189 & 3.00 \\
\hline Segura Jr and Wallace [155] & 1802 & 330 & 229 & 3.140 & 1.300 & 531.0 & 2134 & 2286 & 229 & 0.460 & 0.400 & 578.0 & 48.60 & 703.4 & 184.5 & 3.00 \\
\hline Devi et al. [156] & 0 & 100 & 150 & 1.300 & 1.400 & 400.0 & 3800 & 1000 & 100 & 0.570 & 0.280 & 400.0 & 30.00 & 157.8 & 16.7 & 2.50 \\
\hline Dragan et al. [157] & 0 & 0 & 0 & 0.000 & 0.000 & 500.0 & 1800 & 880 & 240 & 1.190 & 1.280 & 500.0 & 40.00 & 382.0 & 100 & 5.00 \\
\hline Ganesan et al. [158] & 0 & 100 & 100 & 1.130 & 1.130 & 415.0 & 700 & 500 & 60 & 0.570 & 0.180 & 415.0 & 53.00 & 145.0 & 38.5 & 3.60 \\
\hline Ganesan et al. [158] & 0 & 100 & 100 & 1.130 & 1.130 & 415.0 & 700 & 500 & 60 & 0.570 & 0.180 & 415.0 & 53.00 & 152.0 & 30.8 & 4.00 \\
\hline Ganesan et al. [158] & 0 & 130 & 130 & 1.190 & 1.190 & 415.0 & 2200 & 540 & 80 & 0.470 & 0.630 & 415.0 & 60.00 & 53.5 & 4.76 & 2.40 \\
\hline Ganesan et al. [158] & 0 & 130 & 130 & 1.190 & 1.190 & 415.0 & 2200 & 540 & 80 & 0.470 & 0.630 & 415.0 & 60.00 & 55.0 & 5.46 & 2.40 \\
\hline Liu et al. [159] & 400 & 100 & 100 & 8.000 & 0.440 & 4144.0 & 1200 & 700 & 100 & 0.440 & 0.440 & 4144.0 & 48.60 & 297.0 & 188 & 1.83 \\
\hline Liu et al. [159] & 400 & 100 & 100 & 8.000 & 0.660 & 414.0 & 1200 & 700 & 100 & 0.660 & 0.660 & 414.0 & 48.60 & 302.0 & 152 & 1.83 \\
\hline
\end{tabular}


Table A2. Cont.

\begin{tabular}{|c|c|c|c|c|c|c|c|c|c|c|c|c|c|c|c|c|}
\hline Reference & $\begin{array}{l}\text { A.L } \\
\text { kN }\end{array}$ & $\begin{array}{c}\text { ac } \\
\mathrm{mm}\end{array}$ & $\begin{array}{c}\mathrm{bc} \\
\mathrm{mm}\end{array}$ & $\begin{array}{c}\rho_{v c} \\
\%\end{array}$ & $\begin{array}{c}\rho_{h c} \\
\%\end{array}$ & $\begin{array}{l}\text { Fyc } \\
\text { MPa }\end{array}$ & $\begin{array}{l}\mathrm{hw} \\
\mathrm{mm}\end{array}$ & $\begin{array}{l}1 \mathrm{w} \\
\mathrm{mm}\end{array}$ & $\begin{array}{l}\text { tw } \\
\mathrm{mm}\end{array}$ & $\begin{array}{c}\rho_{v w} \\
\%\end{array}$ & $\begin{array}{c}\rho_{h w} \\
\%\end{array}$ & $\begin{array}{l}\text { Fyw } \\
\text { MPa }\end{array}$ & $\begin{array}{l}\text { Fcw } \\
\mathrm{MPa}\end{array}$ & $\begin{array}{c}\mathbf{P} \\
\mathbf{k N}\end{array}$ & $\begin{array}{c}\mathrm{K} \\
\mathrm{kN} / \mathrm{mm}\end{array}$ & $\begin{array}{l}\mathrm{D} \\
\%\end{array}$ \\
\hline Lu et al. [160] & 1200 & 100 & 200 & 1.170 & 0.180 & 552.0 & 2600 & 1500 & 200 & 0.330 & 0.330 & 557.0 & 39.10 & 410.3 & 44.5 & 2.50 \\
\hline Zhu and Guo [161] & 946 & 200 & 400 & 2.000 & 0.400 & 438.0 & 3280 & 1700 & 200 & 0.410 & 0.390 & 451.0 & 35.38 & 682.0 & 38.5 & 2.66 \\
\hline Zhu and Guo [162] & 1220 & 200 & 400 & 2.000 & 0.500 & 438.0 & 3400 & 1700 & 200 & 0.370 & 0.390 & 455.0 & 35.90 & 660.0 & 27 & 2.60 \\
\hline Barda et al. [163] & 0 & 610 & 102 & 1.800 & 1.800 & 535.0 & 952.5 & 1905 & 102 & 0.500 & 0.500 & 554.0 & 29.40 & 1368.4 & 829.73 & 0.61 \\
\hline Barda et al. [163] & 0 & 610 & 102 & 6.400 & 6.400 & 496.0 & 952.5 & 1905 & 102 & 0.500 & 0.500 & 562.4 & 16.60 & 1039.2 & 800.1 & 0.69 \\
\hline Barda et al. [163] & 0 & 610 & 102 & 4.100 & 4.100 & 421.0 & 952.5 & 1905 & 102 & 0.500 & 0.500 & 555.3 & 27.40 & 1193.6 & 1100 & 0.56 \\
\hline Barda et al. [163] & 0 & 610 & 102 & 4.100 & 4.100 & 537.0 & 952.5 & 1905 & 102 & 0.500 & 0.000 & 545.5 & 19.30 & 1097.4 & 1137.9 & 0.53 \\
\hline Barda et al. [163] & 0 & 610 & 102 & 4.100 & 4.100 & 537.0 & 952.5 & 1905 & 102 & 0.000 & 0.500 & 537.0 & 29.40 & 728.9 & 1466.8 & 0.53 \\
\hline Barda et al. [163] & 0 & 610 & 102 & 4.100 & 4.100 & 539.0 & 952.5 & 1905 & 102 & 0.250 & 0.500 & 506.0 & 21.56 & 929.4 & 1148.8 & 0.61 \\
\hline Barda et al. [163] & 0 & 610 & 102 & 4.100 & 4.100 & 549.0 & 476 & 1905 & 102 & 0.500 & 0.500 & 541.3 & 26.10 & 1227.4 & 2933.7 & 0.85 \\
\hline Barda et al. [163] & 0 & 610 & 102 & 4.100 & 4.100 & 498.0 & 1905 & 1905 & 102 & 0.500 & 0.500 & 537.7 & 24.15 & 953.8 & 395.1 & 0.56 \\
\hline Mansur et al. [164] & 0 & 200 & 100 & 3.140 & 0.280 & 460.0 & 600 & 900 & 60 & 0.628 & 0.628 & 327.0 & 33.70 & 397.4 & 172 & 1.40 \\
\hline Mansur et al. [164] & 0 & 200 & 100 & 3.140 & 0.280 & 460.0 & 600 & 900 & 60 & 0.550 & 0.550 & 429.0 & 31.40 & 399.6 & 176.2 & 2.50 \\
\hline Mansur et al. [164] & 0 & 200 & 100 & 3.140 & 0.280 & 460.0 & 600 & 900 & 60 & 0.550 & 0.550 & 429.0 & 31.30 & 378.8 & 217.5 & 2.14 \\
\hline Mansur et al. [164] & 0 & 200 & 100 & 3.140 & 0.280 & 460.0 & 600 & 900 & 60 & 0.620 & 0.620 & 359.0 & 37.40 & 414.3 & 241.7 & 2.12 \\
\hline
\end{tabular}




\section{Appendix B. Predictive Meta-Models}

Table A3. Detailed meta-model for strength response of SPSW.

\begin{tabular}{|c|c|c|c|c|c|c|}
\hline & w11 & w21 & w31 & w41 & w51 & w61 \\
\hline$h_{f}$ & 0.66046 & -0.80899 & 0.42823 & 0.01536 & -0.18274 & 0.56987 \\
\hline$b_{f}$ & -0.14694 & -0.33524 & 0.10281 & -0.21930 & 0.56121 & 0.07946 \\
\hline$A_{c}$ & -0.31230 & 0.58567 & 0.50296 & -0.21616 & 0.09030 & -0.54168 \\
\hline$I_{c}$ & -0.25301 & 0.09989 & -0.84791 & 0.83422 & -0.71050 & -0.52802 \\
\hline$A_{b}$ & -0.12027 & -0.38129 & 0.00603 & -0.30422 & 0.73080 & 0.34905 \\
\hline$I_{b}$ & -0.70756 & 0.10070 & -0.65471 & -0.11693 & -0.02622 & 1.01008 \\
\hline$h_{p}$ & -0.11373 & 0.75990 & -0.05930 & 0.37923 & 0.20590 & -0.37343 \\
\hline$b_{p}$ & 0.87044 & 0.01324 & -0.11518 & -0.65201 & 0.62558 & -0.58151 \\
\hline$t_{p}$ & -0.52584 & 0.63379 & 0.89311 & 0.10456 & -0.50542 & -0.05660 \\
\hline$F_{y p}$ & -0.01419 & 0.86811 & -0.22826 & 0.63826 & 0.29556 & 0.20831 \\
\hline$F_{u p}$ & -0.40961 & -0.39314 & 0.09353 & -0.73352 & 0.19547 & 0.39709 \\
\hline \multirow[t]{2}{*}{$E_{p}$} & 0.27467 & -0.16444 & -0.48999 & -0.37209 & 0.38294 & -0.64189 \\
\hline & b11 & b21 & b31 & b41 & b51 & b61 \\
\hline Bias & -1.63294 & -1.18766 & -0.03826 & -0.16751 & -0.86762 & 1.24930 \\
\hline \multirow[b]{2}{*}{$P$} & w12 & w22 & w32 & w42 & w52 & w62 \\
\hline & -0.12684 & 1.34651 & 0.45059 & -0.06601 & 0.44606 & -0.52102 \\
\hline & b12 & & & & & \\
\hline Bias & 1.02066 & & & & & \\
\hline
\end{tabular}

Table A4. Detailed meta-model for stiffness response of SPSW.

\begin{tabular}{|c|c|c|c|c|c|c|}
\hline & w11 & w21 & w31 & w41 & w51 & w61 \\
\hline$h_{f}$ & 0.37305 & 0.22685 & 0.96172 & 0.27180 & 1.48534 & 0.13780 \\
\hline$b_{f}$ & -0.19722 & -0.32485 & -0.45253 & -0.11746 & -0.60365 & 0.88083 \\
\hline$A_{c}$ & 0.37518 & 0.81240 & 0.39296 & 0.71123 & -0.44507 & -0.83046 \\
\hline$I_{C}$ & -0.79638 & -0.29590 & -0.74050 & -0.30217 & 0.20901 & 0.30874 \\
\hline$A_{b}$ & 0.47524 & -0.30413 & -0.65031 & 0.63964 & -0.24198 & -0.63278 \\
\hline$I_{b}$ & -0.22044 & 0.87375 & -0.38598 & 0.29749 & 0.37306 & 0.22694 \\
\hline$h_{p}$ & -0.14532 & 0.85001 & -1.51534 & -0.48506 & 0.02425 & 0.11618 \\
\hline$b_{p}$ & 0.37593 & 0.02488 & -0.27690 & 0.76170 & 0.88023 & 0.90595 \\
\hline$t_{p}$ & 0.11381 & 0.04305 & -0.37526 & -0.77502 & -0.92189 & -0.21587 \\
\hline$F_{y p}$ & 0.38798 & -0.53169 & 0.14933 & 0.44108 & 0.14424 & 0.79407 \\
\hline$F_{u p}$ & -0.79426 & -0.04613 & 1.40176 & 0.68674 & -0.83376 & 0.50377 \\
\hline \multirow[t]{2}{*}{$E_{p}$} & -0.66830 & 0.11349 & -1.27297 & 0.53022 & -0.02661 & 0.05945 \\
\hline & b11 & b21 & b31 & b41 & b51 & b61 \\
\hline Bias & -0.34198 & -0.20046 & -1.47401 & -0.09315 & -1.27874 & 0.46863 \\
\hline \multirow{3}{*}{$k$} & w12 & w22 & w32 & w42 & w52 & w62 \\
\hline & -0.34198 & -0.20046 & -1.47401 & -0.09315 & -1.27874 & 0.46863 \\
\hline & b12 & & & & & \\
\hline Bias & 0.80271 & & & & & \\
\hline
\end{tabular}


Table A5. Detailed meta-model for strength response of RCSW.

\begin{tabular}{|c|c|c|c|c|c|c|c|c|c|c|}
\hline & w11 & w21 & w31 & w41 & w51 & w61 & w71 & w81 & w91 & w101 \\
\hline A.L & 0.03035 & 0.56271 & 0.65166 & -0.49158 & 0.95894 & -0.25677 & 0.35357 & 1.74573 & 1.36929 & 0.07470 \\
\hline$W_{1 b}$ & 0.00363 & -0.81004 & -0.28891 & 0.49325 & -0.28890 & 0.97131 & -0.02621 & 0.48072 & -0.97056 & 0.24001 \\
\hline$W_{2 b}$ & 0.44522 & -0.27800 & -0.01113 & -0.94223 & -0.04538 & 1.38581 & 0.34507 & -1.18083 & -0.18496 & -0.64199 \\
\hline$\rho_{v c}$ & 0.44214 & 1.67735 & 0.23993 & -0.18993 & -0.11241 & 1.53625 & -0.54095 & -0.00594 & -0.00075 & -0.00729 \\
\hline$\rho_{c h}$ & -0.70002 & -0.62402 & -0.33676 & -0.88147 & 0.72609 & -0.29662 & -0.37476 & 1.07065 & -0.31478 & 1.43449 \\
\hline$F_{y c}$ & -0.21847 & 0.03408 & -0.40615 & 0.58864 & -0.25504 & 0.55362 & -0.19680 & -0.14657 & -0.95686 & 0.22086 \\
\hline$h_{w}$ & 0.96457 & 0.87677 & 1.11667 & 0.39403 & 0.16933 & 0.23444 & 0.41240 & -0.23943 & 0.06403 & 2.21632 \\
\hline$l_{w}$ & 0.75509 & -0.72998 & 0.90047 & -0.78593 & 3.45766 & -1.78285 & -2.39491 & 0.78037 & 0.99762 & -1.44196 \\
\hline$t_{w}$ & 0.23458 & -0.93816 & 0.02132 & -1.07066 & 0.15785 & -1.02085 & -0.88543 & -1.35955 & 1.16582 & -0.01547 \\
\hline$\rho_{v w}$ & -0.44964 & 0.53509 & 0.37030 & 0.44851 & -2.13863 & 0.39363 & -1.01356 & -1.20354 & -0.23381 & -0.72274 \\
\hline$\rho_{h w}$ & 0.46759 & 0.61964 & 0.60364 & 0.60909 & -0.09700 & -0.04887 & -0.08554 & -0.07229 & -0.02111 & -1.10481 \\
\hline$F_{y w}$ & -0.25250 & -0.34851 & -1.11666 & 0.27549 & 0.29564 & 0.62440 & 0.07660 & 1.05904 & -0.28551 & 0.02229 \\
\hline$f_{c}^{\prime}$ & -0.63277 & 0.02735 & 0.54041 & -1.64195 & 0.07042 & 0.27903 & -0.17193 & -0.89626 & -0.00433 & -0.17178 \\
\hline & b11 & b21 & b31 & b41 & b51 & b61 & b71 & b81 & b91 & b101 \\
\hline Bias & 1.54250 & -1.05560 & -0.64521 & -0.27120 & -0.56445 & 0.34340 & 0.67124 & -0.89740 & -1.64937 & -1.19042 \\
\hline & w12 & w22 & w32 & w42 & w52 & w62 & w72 & w82 & w92 & w102 \\
\hline$P$ & 0.80903 & -1.09306 & 0.46271 & -0.15223 & -1.58711 & 0.42260 & -1.19451 & 0.19695 & 0.20121 & -0.56320 \\
\hline & b12 & & & & & & & & & \\
\hline Bias & 0.27163 & & & & & & & & & \\
\hline
\end{tabular}

Table A6. Detailed meta-model for stiffness response of RCSW.

\begin{tabular}{|c|c|c|c|c|c|c|c|c|c|c|}
\hline & w11 & w21 & w31 & w41 & w51 & w61 & w71 & w81 & w91 & w101 \\
\hline A.L & 0.27836 & 0.34179 & 0.55152 & 0.79469 & 1.25982 & 0.90687 & -0.02805 & 0.79850 & 0.09437 & -0.06686 \\
\hline$W_{1 b}$ & -0.35504 & -0.29662 & 0.63076 & 1.04187 & 0.28381 & -1.12608 & 0.60906 & 0.77589 & 0.17191 & -0.05209 \\
\hline$W_{2 b}$ & 0.38845 & 0.06067 & -0.07384 & -0.10242 & 0.11835 & 0.68601 & -0.51639 & 0.69812 & -0.33039 & -0.81421 \\
\hline$\rho_{v c}^{2 v}$ & 0.00685 & -0.42597 & -0.56353 & -0.27964 & 0.07599 & 0.48414 & -0.13054 & 0.28632 & -0.56540 & 0.18407 \\
\hline$\rho_{c h}$ & -0.18496 & -0.93420 & -0.21477 & -1.30458 & -0.25130 & -0.16112 & 1.17348 & -0.46040 & -1.01113 & -0.93252 \\
\hline$F_{y c}$ & -0.32846 & 0.19449 & -0.00322 & -0.23876 & 0.00709 & -0.24757 & 0.12753 & -0.93046 & 0.73564 & -1.19427 \\
\hline$h_{w}$ & -2.48463 & -0.53283 & -0.53211 & -0.16902 & -1.33119 & -0.02211 & 0.01571 & -0.43720 & -1.38712 & 1.51850 \\
\hline$l_{w}$ & 0.33724 & 0.90620 & 0.57674 & 0.18346 & -0.22445 & 0.17460 & -0.49526 & -0.55005 & 0.45015 & 0.01144 \\
\hline$t_{w}$ & -0.05230 & 0.71080 & -0.49130 & -0.72176 & 0.15461 & -0.04615 & 0.24655 & -0.73673 & 0.44326 & -0.53894 \\
\hline$\rho_{v w}$ & 0.19946 & 1.35835 & -0.56388 & 0.44006 & -0.21862 & -0.43304 & -0.10659 & 1.16227 & -0.28689 & 0.63142 \\
\hline$\rho_{h w}$ & 0.56298 & 0.40776 & 0.68201 & -0.37318 & -0.10458 & 0.18270 & -0.76181 & -0.20352 & 0.46643 & 0.20279 \\
\hline$F_{y w}$ & 0.12877 & -0.02556 & 0.16279 & 0.55430 & -0.10325 & -0.35100 & 1.00406 & -0.47198 & 0.09076 & -0.07678 \\
\hline \multirow[t]{2}{*}{$f_{c}^{\prime}$} & -0.25932 & -0.90033 & 0.23902 & 0.28550 & 0.11517 & 0.17102 & -0.33770 & -0.05328 & 0.31861 & -0.72958 \\
\hline & b11 & b21 & b31 & b41 & b51 & b61 & b71 & b81 & b91 & b101 \\
\hline \multirow[t]{2}{*}{ Bias } & -2.40062 & -1.41379 & 1.54715 & -0.57911 & 0.65255 & -0.61406 & -0.48502 & 1.07819 & 1.26215 & 4.07677 \\
\hline & w12 & w22 & w32 & w42 & w52 & w62 & w72 & w82 & w92 & w102 \\
\hline \multirow[t]{2}{*}{$K$} & 1.22667 & 0.59476 & -0.31743 & 0.59542 & 1.08410 & -0.91750 & -1.24778 & -0.90334 & -1.04493 & -1.19961 \\
\hline & b12 & & & & & & & & & \\
\hline Bias & 0.23727 & & & & & & & & & \\
\hline
\end{tabular}

Table A7. Detailed meta-model for drift response of RCSW.

\begin{tabular}{|c|c|c|c|c|c|c|c|c|c|c|}
\hline & w11 & w21 & w31 & w41 & w51 & w61 & w71 & w81 & w91 & w101 \\
\hline A.L & -0.38551 & -0.21555 & 0.24561 & 0.72432 & 0.22761 & -0.32669 & -1.22827 & 1.02986 & 0.29691 & -0.65452 \\
\hline$W_{1 b}$ & -0.99673 & 0.44528 & -2.90900 & -0.04773 & 0.07769 & 0.14600 & -0.41686 & -2.04320 & -0.04020 & -0.30586 \\
\hline$W_{2 b}$ & -0.46010 & 0.27508 & -1.58672 & -0.07816 & -1.17859 & -0.02286 & 0.65862 & 1.95612 & -0.39298 & -0.07518 \\
\hline$\rho_{v c}$ & 0.87950 & 1.24468 & 0.58189 & 0.45051 & -0.09522 & -1.02249 & -0.83121 & 0.20119 & 0.95248 & -0.00905 \\
\hline$\rho_{c h}$ & -0.12796 & 0.88787 & -1.25140 & -0.33128 & -0.95154 & -1.33210 & -0.34843 & 1.30857 & 0.56996 & -0.77308 \\
\hline$F_{y c}$ & -0.79125 & 0.59283 & 0.61220 & 0.65224 & -0.79856 & -0.22531 & 0.88950 & -0.53688 & -0.20737 & 0.09254 \\
\hline$h_{w}$ & 1.01985 & 0.11646 & 0.07401 & 0.91305 & -0.10624 & -0.59715 & 0.53518 & 0.48895 & -0.48217 & -1.09651 \\
\hline$l_{w}$ & -0.81903 & 0.56432 & -0.97381 & 0.57371 & 0.29790 & -1.10196 & -0.38007 & -0.45571 & 0.35466 & -0.85581 \\
\hline$t_{w}$ & 0.90861 & -0.48538 & 0.62197 & 0.16745 & -0.22928 & 0.46233 & 0.01534 & 0.57427 & -0.45866 & 0.40609 \\
\hline$\rho_{v w}$ & 0.14697 & -0.92753 & 0.61884 & 0.57854 & 0.93538 & -0.50672 & -0.20537 & -0.47842 & 1.45274 & -0.04762 \\
\hline$\rho_{h w}$ & 1.19628 & 0.57915 & 0.40955 & 0.45958 & 0.11556 & -0.85086 & -0.68757 & -0.31959 & 1.00650 & 0.12659 \\
\hline$F_{y w}$ & -0.02750 & 1.08842 & -1.35543 & -0.12400 & 0.09936 & -0.50841 & 0.31056 & -0.21224 & -0.15709 & 0.27799 \\
\hline$f_{c}^{\prime}$ & 0.22400 & -1.75419 & -1.45567 & -0.30999 & 0.52838 & -0.35201 & 0.33484 & -0.72868 & 0.55217 & 0.18422 \\
\hline & b11 & b21 & b31 & b41 & b51 & b61 & b71 & b81 & b91 & b101 \\
\hline Bias & -1.62011 & 0.13056 & -0.50546 & -0.75766 & -0.61882 & -0.24612 & 0.63451 & 0.91834 & -1.27594 & -1.70098 \\
\hline & w12 & w22 & w32 & w42 & w52 & w62 & w72 & w82 & w92 & w102 \\
\hline$D$ & 1.38455 & -0.54226 & 0.41286 & -0.04294 & -1.17546 & 0.35975 & -0.26591 & -0.71351 & -0.36289 & -0.31776 \\
\hline Bias & $\begin{array}{c}\text { b12 } \\
0.16445\end{array}$ & & & & & & & & & \\
\hline
\end{tabular}




\section{References}

1. Bertero, V.V. The Response of Shear Walls Subjected to Dynamic Loads. Ph.D. Thesis, Massachusetts Institute of Technology, Cambridge, MA, USA, 1957.

2. Hidalgo, P.A.; Ledezma, C.A.; Jordan, R.M. Seismic behavior of squat reinforced concrete shear walls. Earthq. Spectra 2002, 18, 287-308. [CrossRef]

3. Bao, Y.; Kunnath, S.K. Simplified progressive collapse simulation of RC frame-wall structures. Eng. Struct. 2010, 32, 3153-3162. [CrossRef]

4. Kolozvari, K.; Wallace, J.W. Practical nonlinear modeling of reinforced concrete structural walls. J. Struct. Eng. 2016, 142, G4016001. [CrossRef]

5. Thorburn, L.J.; Montgomery, C.; Kulak, G.L. Analysis of Steel Plate Shear Walls; Technical Report; University of Alberta: Edmonton, AB, Canada, 1983.

6. Jalali, S.; Banazadeh, M. Development of a new deteriorating hysteresis model for seismic collapse assessment of thin steel plate shear walls. Thin-Walled Struct. 2016, 106, 244-257. [CrossRef]

7. Yokel, F.Y.; Fattal, S.G. Failure hypothesis for masonry shear walls. J. Struct. Div. 1976, 102, 515-532.

8. Caliò, I.; Pantò, B. A macro-element modelling approach of Infilled Frame Structures. Comput. Struct. 2014, 143, 91-107. [CrossRef]

9. Pantò, B.; Cannizzaro, F.; Caddemi, S.; Caliò, I. 3D macro-element modelling approach for seismic assessment of historical masonry churches. Adv. Eng. Softw. 2016, 97, 40-59. [CrossRef]

10. Bahmani, P.; van de Lindt, J.W. Experimental and numerical assessment of woodframe sheathing layer combinations for use in strength-based and performance-based design. J. Struct. Eng. 2014, 142, E4014001. [CrossRef]

11. Stitic, A.; Nguyen, A.C.; Rezaei Rad, A.; Weinand, Y. Numerical Simulation of the Semi-Rigid Behaviour of Integrally Attached Timber Folded Surface Structures. Buildings 2019, 9, 55. [CrossRef]

12. Timler, P.A.; Kulak, G.L. Experimental Study of Steel Plate Shear Walls; Technical Report; University of Alberta: Edmonton, AB, Canada, 1983.

13. Tromposch, E.W.; Kulak, G.L. Cyclic and Static Behaviour of Thin Panel Steel Plate Shear Walls; Technical Report; University of Alberta: Edmonton, AB, Canada, 1987.

14. Timler, P. Economical design of steel plate shear walls from a consulting engineers perspective. In 1999 North American Steel Construction Conference (NASCC); American Institute of Steel Construction: Chicago, IL, USA, 1999; p. 36.

15. Hajimirsadeghi, M.; Mirtaheri, M.; Zandi, A.; Hariri-Ardebili, M. Experimental cyclic test and failure modes of a full scale enhanced modular steel plate shear wall. Eng. Fail. Anal. 2019, 95, 283-288. [CrossRef]

16. AISC. Load and Resistance Factor Design Specifications for Structural Steel Buildings; American Institute of Steel Construction: Chicago, IL, USA, 1999.

17. CSA-S16-01. Limit States Design of Steel Structures; Canadian Standard Association: Toronto, ON, Canada, 2001.

18. Marzban, S.; Almasi, A.; Schwarz, J. Reinforced Concrete Structural Wall Database Development for Model Validation. In Proceedings of the 2nd European Conference on Earthquake Engineering and Seismology, Istanbul, Turkey, 25-29 August 2014.

19. Hariri-Ardebili, M.A.; Saouma, V.E. Sensitivity and uncertainty analysis of AAR affected reinforced concrete shear walls. Eng. Struct. 2018, 172, 334-345. [CrossRef]

20. Salazar, F.; Morán, R.; Toledo, M.Á.; Oñate, E. Data-based models for the prediction of dam behaviour: A review and some methodological considerations. Arch. Comput. Methods Eng. 2017, 24, 1-21. [CrossRef]

21. Hariri-Ardebili, M.A.; Pourkamali-Anaraki, F. Support vector machine based reliability analysis of concrete dams. Soil Dyn. Earthq. Eng. 2018, 104, 276-295. [CrossRef]

22. Blatman, G.; Sudret, B. Adaptive sparse polynomial chaos expansion based on least angle regression. J. Comput. Phys. 2011, 230, 2345-2367. [CrossRef]

23. Du, K.L.; Swamy, M.N. Neural Networks in a Softcomputing Framework; Springer Science \& Business Media: London, UK, 2006.

24. McCulloch, W.S.; Pitts, W. A logical calculus of the ideas immanent in nervous activity. Bull. Math. Biophys. 1943, 5, 115-133. [CrossRef]

25. Hebb, D. The Organization of Behavior; Wiley: New York, NY, USA, 1949. 
26. Widrow, B.; Hoff, M.E. Adaptive Switching Circuits; Technical Report; Stanford Univ CA Stanford Electronics Labs: Stanford, CA, USA, 1960.

27. Rosenblatt, F. Principles of Neurodynamics. Perceptrons and the Theory of Brain Mechanisms; Technical Report; Cornell Aeronautical Lab Inc.: Buffalo, NY, USA, 1961.

28. Rumelhart, D.E.; Hinton, G.E.; Williams, R.J. Learning Internal Representations by Error Propagation; Technical Report; California Univ San Diego La Jolla Inst for Cognitive Science: San Diego, CA, USA, 1985.

29. Fausett, L.V. Fundamentals of Neural Networks: Architectures, Algorithms, and Applications; Prentice-Hall: Englewood Cliffs, NJ, USA, 1994; Volume 3.

30. Cybenko, G. Approximation by superpositions of a sigmoidal function. Math. Control Signals Syst. 1989, 2, 303-314. [CrossRef]

31. Funahashi, K.I. On the approximate realization of continuous mappings by neural networks. Neural Netw. 1989, 2, 183-192. [CrossRef]

32. Hornik, K.; Stinchcombe, M.; White, H. Multilayer feedforward networks are universal approximators. Neural Netw. 1989, 2, 359-366. [CrossRef]

33. Xiang, C.; Ding, S.Q.; Lee, T.H. Geometrical interpretation and architecture selection of MLP. IEEE Trans. Neural Netw. 2005, 16, 84-96. [CrossRef]

34. Cybenko, G. Continuous Valued Neural Networks with Two Hidden Layers Are Sufficient; Technical Report; Department of Computer Science, Tufts University: Medford, MA, USA, 1988.

35. Bui, T.Q.; Tran, A.V.; Shah, A.A. Improved knowledge-based neural network (KBNN) model for predicting spring-back angles in metal sheet bending. Int. J. Model. Simul. Sci. Comput. 2014, 5, 1350026. [CrossRef]

36. Chester, D.L. Why Two Hidden Layers Are Better than One; Proc. IJCNN: Washington, DC, USA, 1990; Volume 1, pp. 265-268.

37. Adeli, H.; Seon Park, H. Counterpropagation neural networks in structural engineering. J. Struct. Eng. 1995, 121, 1205-1212. [CrossRef]

38. Xu, Y.; Liu, G.; Wu, Z.; Huang, X. Adaptive multilayer perceptron networks for detection of cracks in anisotropic laminated plates. Int. J. Solids Struct. 2001, 38, 5625-5645. [CrossRef]

39. De Lima, L.; Vellasco, P.D.S.; De Andrade, S.; Da Silva, J.; Vellasco, M. Neural networks assessment of beam-to-column joints. J. Braz. Soc. Mech. Sci. Eng. 2005, 27, 314-324. [CrossRef]

40. Abdalla, J.A.; Elsanosi, A.; Abdelwahab, A. Modeling and simulation of shear resistance of R/C beams using artificial neural network. J. Frankl. Inst. 2007, 344, 741-756. [CrossRef]

41. Kumar, R.; Mishra, B.; Jain, S. Vibration control of smart composite laminated spherical shell using neural network. J. Intell. Mater. Syst. Struct. 2008, 19, 947-957. [CrossRef]

42. González, M.P.; Zapico, J.L. Seismic damage identification in buildings using neural networks and modal data. Comput. Struct. 2008, 86, 416-426. [CrossRef]

43. Yan, B.; Cui, Y.; Zhang, L.; Zhang, C.; Yang, Y.; Bao, Z.; Ning, G. Beam structure damage identification based on BP neural network and support vector machine. Math. Probl. Eng. 2014, 2014, 850141. [CrossRef]

44. Lee, S.; Lee, C. Prediction of shear strength of FRP-reinforced concrete flexural members without stirrups using artificial neural networks. Eng. Struct. 2014, 61, 99-112. [CrossRef]

45. Asteris, P.G.; Plevris, V. Anisotropic masonry failure criterion using artificial neural networks. Neural Comput. Appl. 2017, 28, 2207-2229. [CrossRef]

46. Toghroli, A.; Suhatril, M.; Ibrahim, Z.; Safa, M.; Shariati, M.; Shamshirband, S. Potential of soft computing approach for evaluating the factors affecting the capacity of steel-concrete composite beam. J. Intell. Manuf. 2018, 29, 1793-1801. [CrossRef]

47. Naderpour, H.; Poursaeidi, O.; Ahmadi, M. Shear resistance prediction of concrete beams reinforced by FRP bars using artificial neural networks. Measurement 2018, 126, 299-308. [CrossRef]

48. Rezaei Rad, A.; Banazadeh, M. Probabilistic risk-based performance evaluation of seismically base-isolated steel structures subjected to far-field earthquakes. Buildings 2018, 8, 128. [CrossRef]

49. Ghorbani, A.; Hasanzadehshooiili, H.; Sadowski, Ł. Neural Prediction of Tunnels' Support Pressure in Elasto-Plastic, Strain-Softening Rock Mass. Appl. Sci. 2018, 8, 841. [CrossRef]

50. Asteris, P.G.; Kolovos, K.G. Self-compacting concrete strength prediction using surrogate models. Neural Comput. Appl. 2019, 31, 409-424. [CrossRef]

51. Chen, H.; Asteris, P.G.; Jahed Armaghani, D.; Gordan, B.; Pham, B.T. Assessing Dynamic Conditions of the Retaining Wall: Developing Two Hybrid Intelligent Models. Appl. Sci. 2019, 9, 1042. [CrossRef] 
52. Asteris, P.G.; Nikoo, M. Artificial bee colony-based neural network for the prediction of the fundamental period of infilled frame structures. Neural Comput. Appl. 2019, 1-11. [CrossRef]

53. Li, H.; Chen, C.P.; Huang, H.P. Fuzzy Neural Intelligent Systems: Mathematical Foundation and the Applications in Engineering; CRC Press: Boca Raton, FL, USA, 2000.

54. Thimm, G.; Fiesler, E. High-order and multilayer perceptron initialization. IEEE Trans. Neural Netw. 1997, 8, 349-359. [CrossRef]

55. Hirose, Y.; Yamashita, K.; Hijiya, S. Back-propagation algorithm which varies the number of hidden units. Neural Netw. 1991, 4, 61-66. [CrossRef]

56. Ozturan, M.; Kutlu, B.; Ozturan, T. Comparison of concrete strength prediction techniques with artificial neural network approach. Build. Res. J. 2008, 56, 23-36.

57. Hecht-Nielsen, R. Kolmogorov's mapping neural network existence theorem. In Proceedings of the International Conference on Neural Networks; IEEE Press: New York, NY, USA, 1987; Volume 3, pp. 11-14.

58. Hush, D.R. Classification with neural networks: A performance analysis. In Proceedings of the IEEE International Conference on Systems Engineering, Fairborn, OH, USA, 24-26 August 1989; pp. 277-280.

59. Ripley, B.D. Statistical aspects of neural networks. Netw. Chaos Stat. Probab. Asp. 1993, 50, 40-123.

60. Gallant, S.I.; Gallant, S.I. Neural Network Learning and Expert Systems; MIT Press: Cambridge, MA, USA, 1993.

61. Wang, C. A Theory of Generalization in Learning Machines with Neural Network Applications. Ph.D. Thesis, University of Pennsylvania, Philadelphia, PA, USA, 1994.

62. Masters, T. Practical Neural Network Recipes in C++; Morgan Kaufmann: Burlington, MA, USA, 1993.

63. Paola, J. Neural Network Classification of Multispectral Imagery. Master's Thesis, The University of Arizona, Tucson, AZ, USA, 1994.

64. Li, J.Y.; Chow, T.W.; Yu, Y.L. The estimation theory and optimization algorithm for the number of hidden units in the higher-order feedforward neural network. In Proceedings of the ICNN'95-International Conference on Neural Networks, Perth, WA, Australia, 27 November-1 December 1995; Volume 3, pp. 1229-1233.

65. Tamura, S.; Tateishi, M. Capabilities of a four-layered feedforward neural network: four layers versus three. IEEE Trans. Neural Netw. 1997, 8, 251-255. [CrossRef] [PubMed]

66. Lai, S.; Serra, M. Concrete strength prediction by means of neural network. Constr. Build. Mater. 1997, 11, 93-98. [CrossRef]

67. Nagendra, S. Practical Aspects of Using Neural Networks: Necessary Preliminary Specifications; Technical Paper; GE Research and Development Center: Niskayuna, NY, USA, 1998.

68. Gencay, R. Linear, non-linear and essential foreign exchange rate prediction with simple technical trading rules. J. Int. Econ. 1999, 47, 91-107. [CrossRef]

69. Chris Wong, C.C.; Chan, M.C.; Lam, C.C. Financial Time Series Forecasting By Neural Network Using Conjugate Gradient Learning Algorithm And Multiple Linear Regression Weight Initialization. Soc. Comput. Econ. Comput. Econ. Financ. 2000, 61, 326-342.

70. Heaton, J. Artificial Intelligence for Humans. Volume 1: Fundamental Algorithms; CreateSpace Independent Publishing: North Charleston, SC, USA, 2013.

71. Zhang, Z.; Ma, X.; Yang, Y. Bounds on the number of hidden neurons in three-layer binary neural networks. Neural Netw. 2003, 16, 995-1002. [CrossRef]

72. Huang, G.B. Learning capability and storage capacity of two-hidden-layer feedforward networks. IEEE Trans. Neural Netw. 2003, 14, 274-281. [CrossRef] [PubMed]

73. Ke, J.; Liu, X. Empirical analysis of optimal hidden neurons in neural network modeling for stock prediction. In Proceedings of the 2008 IEEE Pacific-Asia Workshop on Computational Intelligence and Industrial Application, Wuhan, China, 19-20 December 2008; Volume 2, pp. 828-832.

74. Trenn, S. Multilayer perceptrons: Approximation order and necessary number of hidden units. IEEE Trans. Neural Netw. 2008, 19, 836-844. [CrossRef] [PubMed]

75. Shibata, K.; Ikeda, Y. Effect of number of hidden neurons on learning in large-scale layered neural networks. In Proceedings of the 2009 ICCAS-SICE, Fukuoka, Japan, 18-21 August 2009; pp. 5008-5013.

76. Hunter, D.; Yu, H.; Pukish, M.; Kolbusz, J.; Wilamowski, B. Selection of Proper Neural Network Sizes and Architectures-A Comparative Study. IEEE Trans. Ind. Inform. 2012, 8, 228-240. [CrossRef]

77. Sheela, K.G.; Deepa, S.N. Review on methods to fix number of hidden neurons in neural networks. Math. Probl. Eng. 2013, 2013, 425740. [CrossRef]

78. Garson, G. Interpreting Neural-Network Connection Strengths. AI Expert 1991, 6, 47-51. 
79. Goh, A. Back-Propagation Neural Networks for Modeling Complex Systems. Artif. Intell. Eng. 1995, 9, 143-151. [CrossRef]

80. Behbahanifard, M.R.; Grondin, G.Y.; Elwi, A.E.A. Experimental and Numerical Investigation of Steel Plate Shear Walls; Department of Civil and Environmental Engineering, University of Alberta: Edmonton, AB, Canada, 2003.

81. Sigariyazd, M.A.; Joghataie, A.; Attari, N.K. Analysis and design recommendations for diagonally stiffened steel plate shear walls. Thin-Walled Struct. 2016, 103, 72-80. [CrossRef]

82. Shekastehband, B.; Azaraxsh, A.; Showkati, H.; Pavir, A. Behavior of semi-supported steel shear walls: Experimental and numerical simulations. Eng. Struct. 2017, 135, 161-176. [CrossRef]

83. Berman, J.W.; Celik, O.C.; Bruneau, M. Comparing hysteretic behavior of light-gauge steel plate shear walls and braced frames. Eng. Struct. 2005, 27, 475-485. [CrossRef]

84. Yu, J.G.; Feng, X.T.; Li, B.; Hao, J.P. Cyclic performance of cross restrained steel plate shear walls with transverse braces. Thin-Walled Struct. 2018, 132, 250-264. [CrossRef]

85. Choi, I.R.; Park, H.G. Ductility and energy dissipation capacity of shear-dominated steel plate walls. J. Struct. Eng. 2008, 134, 1495-1507. [CrossRef]

86. Wang, M.; Shi, Y.; Xu, J.; Yang, W.; Li, Y. Experimental and numerical study of unstiffened steel plate shear wall structures. J. Constr. Steel Res. 2015, 112, 373-386. [CrossRef]

87. Sabouri-Ghomi, S.; Sajjadi, S.R.A. Experimental and theoretical studies of steel shear walls with and without stiffeners. J. Constr. Steel Res. 2012, 75, 152-159. [CrossRef]

88. Chen, S.J.; Jhang, C. Experimental study of low-yield-point steel plate shear wall under in-plane load. J. Constr. Steel Res. 2011, 67, 977-985. [CrossRef]

89. Nateghi-Alahi, F.; Khazaei-Poul, M. Experimental study of steel plate shear walls with infill plates strengthened by GFRP laminates. J. Constr. Steel Res. 2012, 78, 159-172. [CrossRef]

90. Caccese, V.; Elgaaly, M.; Chen, R. Experimental study of thin steel-plate shear walls under cyclic load. J. Struct. Eng. 1993, 119, 573-587. [CrossRef]

91. Alavi, E.; Nateghi, F. Experimental study on diagonally stiffened steel plate shear walls with central perforation. J. Constr. Steel Res. 2013, 89, 9-20. [CrossRef]

92. Park, H.G.; Kwack, J.H.; Jeon, S.W.; Kim, W.K.; Choi, I.R. Framed steel plate wall behavior under cyclic lateral loading. J. Struct. Eng. 2007, 133, 378-388. [CrossRef]

93. Lubell, A.S.; Prion, H.G.; Ventura, C.E.; Rezai, M. Unstiffened steel plate shear wall performance under cyclic loading. J. Struct. Eng. 2000, 126, 453-460. [CrossRef]

94. Roberts, T.M.; Sabouri-Ghomi, S. Hysteretic characteristics of unstiffened perforated steel plate shear panels. Thin-Walled Struct. 1992, 14, 139-151. [CrossRef]

95. Carrillo, J.; Lizarazo, J.M.; Bonett, R. Effect of lightweight and low-strength concrete on seismic performance of thin lightly-reinforced shear walls. Eng. Struct. 2015, 93, 61-69. [CrossRef]

96. Yuan, W.; Zhao, J.; Sun, Y.; Zeng, L. Experimental study on seismic behavior of concrete walls reinforced by PC strands. Eng. Struct. 2018, 175, 577-590. [CrossRef]

97. Shiga, T.; Shibata, A.; Takahashi, J. Experimental study on dynamic properties of reinforced concrete shear walls. In Proceedings of the Fifth World Conference on Earthquake Engineering, Rome, Italy, 25-29 June 1973; Volume 1, pp. 1157-1166.

98. Greifenhagen, C.; Lestuzzi, P. Static cyclic tests on lightly reinforced concrete shear walls. Eng. Struct. 2005, 27, 1703-1712. [CrossRef]

99. Looi, D.; Su, R.; Cheng, B.; Tsang, H. Effects of axial load on seismic performance of reinforced concrete walls with short shear span. Eng. Struct. 2017, 151, 312-326. [CrossRef]

100. Lopes, M. Experimental shear-dominated response of RC walls. Part II: Discussion of results and design implications. Eng. Struct. 2001, 23, 564-574. [CrossRef]

101. Yanez, F.; Park, R.; Paulay, T. Seismic behaviour of reinforced concrete structural walls with irregular openings. In Proceedings of the Pacific Conference on Earthquake Engineering, Auckland, New Zealand, 20-23 November 1991; pp. 3303-3308.

102. Bouchon, M.; Orbovic, N.; Foure, N. Tests on reinforced concrete low-rise shear walls under static cyclic loading. In Proceedings of the 13th World Conference on Earthquake Engineering, Vancouver, BC, Canada, 1-6 August 2004; p. 10.

103. Tasnimi, A. Strength and deformation of mid-rise shear walls under load reversal. Eng. Struct. 2000, 22, 311-322. [CrossRef] 
104. Lefas, I.D.; Kotsovos, M.D.; Ambraseys, N.N. Behavior of reinforced concrete structural walls: Strength, deformation characteristics, and failure mechanism. Struct. J. 1990, 87, 23-31.

105. Lefas, I.D.; Kotsovos, M.D. Strength and deformation characteristics of reinforced concrete walls under load reversals. Struct. J. 1990, 87, 716-726.

106. Pilakoutas, K.; Elnashai, A. Cyclic behavior of reinforced concrete cantilever walls, Part I: Experimental results. ACI Struct. J. 1995, 92, 271-281.

107. Gupta, A.; Rangan, B.V. High-strength concrete (HSC) structural walls. Struct. J. 1998, 95, 194-204.

108. Mickleborough, N.C.; Ning, F.; Chan, C.M. Prediction of stiffness of reinforced concrete shearwalls under service loads. Struct. J. 1999, 96, 1018-1026.

109. Sittipunt, C.; Wood, S.L.; Lukkunaprasit, P.; Pattararattanakul, P. Cyclic behavior of reinforced concrete structural walls with diagonal web reinforcement. Struct. J. 2001, 98, 554-562.

110. Zhang, Y.; Wang, Z. Seismic behavior of reinforced concrete shear walls subjected to high axial loading. Struct. J. 2000, 97, 739-750.

111. Adebar, P.; Ibrahim, A.M.; Bryson, M. Test of high-rise core wall: Effective stiffness for seismic analysis. ACI Struct. J. 2007, 104, 549.

112. Terzioglu, T.; Orakcal, K.; Massone, L.M. Cyclic lateral load behavior of squat reinforced concrete walls. Eng. Struct. 2018, 160, 147-160. [CrossRef]

113. Abdulridha, A.; Palermo, D. Behaviour and modelling of hybrid SMA-steel reinforced concrete slender shear wall. Eng. Struct. 2017, 147, 77-89. [CrossRef]

114. Qazi, S.; Michel, L.; Ferrier, E. Seismic behaviour of RC short shear wall strengthened with externally bonded CFRP strips. Compos. Struct. 2019, 211, 390-400. [CrossRef]

115. Christidis, K.I.; Trezos, K.G. Experimental investigation of existing non-conforming RC shear walls. Eng. Struct. 2017, 140, 26-38. [CrossRef]

116. Wang, W.; Wang, Y.; Lu, Z. Experimental study on seismic behavior of steel plate reinforced concrete composite shear wall. Eng. Struct. 2018, 160, 281-292. [CrossRef]

117. Ren, F.; Chen, J.; Chen, G.; Guo, Y.; Jiang, T. Seismic behavior of composite shear walls incorporating concrete-filled steel and FRP tubes as boundary elements. Eng. Struct. 2018, 168, 405-419. [CrossRef]

118. Le Nguyen, K.; Brun, M.; Limam, A.; Ferrier, E.; Michel, L. Pushover experiment and numerical analyses on CFRP-retrofit concrete shear walls with different aspect ratios. Compos. Struct. 2014, 113, 403-418. [CrossRef]

119. Choi, C.S. Improvement of earthquake-resistant performance of squat shear walls under reversed cyclic loads. Key Eng. Mater. 2006, 324, 535-538. [CrossRef]

120. Cortés-Puentes, W.L.; Palermo, D. Performance of pre-1970s squat reinforced concrete shear walls. Can. J. Civ. Eng. 2018, 45, 922-935. [CrossRef]

121. Cortés-Puentes, W.L.; Palermo, D. Seismic retrofit of concrete shear walls with SMA tension braces. J. Struct. Eng. 2017, 144, 04017200. [CrossRef]

122. Cortés-Puentes, W.L.; Palermo, D. Modeling of RC shear walls retrofitted with steel plates or FRP sheets. J. Struct. Eng. 2011, 138, 602-612. [CrossRef]

123. Qiao, Q.; Cao, W.; Qian, Z.; Li, X.; Zhang, W.; Liu, W. Cyclic behavior of low rise concrete shear walls containing recycled coarse and fine aggregates. Materials 2017, 10, 1400. [CrossRef] [PubMed]

124. Blandon, C.A.; Arteta, C.A.; Bonett, R.L.; Carrillo, J.; Beyer, K.; Almeida, J.P. Response of thin lightly-reinforced concrete walls under cyclic loading. Eng. Struct. 2018, 176, 175-187. [CrossRef]

125. Christidis, K.I.; Vougioukas, E.; Trezos, K.G. Strengthening of non-conforming RC shear walls using different steel configurations. Eng. Struct. 2016, 124, 258-268. [CrossRef]

126. Liao, F.Y.; Han, L.H.; Tao, Z. Performance of reinforced concrete shear walls with steel reinforced concrete boundary columns. Eng. Struct. 2012, 44, 186-209. [CrossRef]

127. Dazio, A.; Beyer, K.; Bachmann, H. Quasi-static cyclic tests and plastic hinge analysis of RC structural walls. Eng. Struct. 2009, 31, 1556-1571. [CrossRef]

128. Zhi, Q.; Guo, Z.; Xiao, Q.; Yuan, F.; Song, J. Quasi-static test and strut-and-tie modeling of precast concrete shear walls with grouted lap-spliced connections. Constr. Build. Mater. 2017, 150, 190-203. [CrossRef]

129. Shen, D.; Yang, Q.; Jiao, Y.; Cui, Z.; Zhang, J. Experimental investigations on reinforced concrete shear walls strengthened with basalt fiber-reinforced polymers under cyclic load. Constr. Build. Mater. 2017, 136, 217-229. [CrossRef] 
130. Zhang, J.W.; Zheng, W.B.; Cao, W.L.; Dong, H.Y.; Li, W.D. Seismic Behavior of Low-rise Concrete Shear Wall with Single Layer of Web Reinforcement and Inclined Rebars: Restoring Force Model. KSCE J. Civ. Eng. 2019, 23, 1302-1319. [CrossRef]

131. Ji, X.; Cheng, X.; Xu, M. Coupled axial tension-shear behavior of reinforced concrete walls. Eng. Struct. 2018, 167, 132-142. [CrossRef]

132. Mohamed, N.; Farghaly, A.S.; Benmokrane, B.; Neale, K.W. Numerical simulation of mid-rise concrete shear walls reinforced with GFRP bars subjected to lateral displacement reversals. Eng. Struct. 2014, 73, 62-71. [CrossRef]

133. Quiroz, L.G.; Maruyama, Y.; Zavala, C. Cyclic behavior of thin RC Peruvian shear walls: Full-scale experimental investigation and numerical simulation. Eng. Struct. 2013, 52, 153-167. [CrossRef]

134. Salonikios, T.; Kappos, A.; Tegos, I.A.; Penelis, G. Cyclic Load Behavior of Low-slenderness R/C Walls: Design Basis and Test Results. ACI Struct. J. 1999, 4, 649-660.

135. Thomsen, J.H. Displacement Based Design of Reinforced Concrete Structural Walls: An Experimental Investigation of Walls with Rectangular and t-Shaped Cross-Sections: A Dissertation. Ph.D. Thesis, Clarkson University Richmond, Ontario County, NY, USA, 1995.

136. Tran, T.A.; Wallace, J. Experimental study of nonlinear flexural and shear deformations of reinforced concrete structural walls. In Proceedings of the 15th World Conference on Earthquake Engineering, Lisbon, Portugal, 24-28 September 2012.

137. Layssi, H.; Mitchell, D. Experiments on seismic retrofit and repair of reinforced concrete shear walls. In Proceedings of the 6th International Conference on FRP Composites in Civil Engineering (CICE), Rome, Italy, 13-15 June 2012; pp. 13-15.

138. Ghorbani-Renani, I.; Velev, N.; Tremblay, R.; Palermo, D.; Massicotte, B.; Léger, P. Modeling and Testing Influence of Scaling Effects on Inelastic Response of Shear Walls. ACI Struct. J. 2009, 106, 358-367.

139. Ghazizadeh, S.; Cruz-Noguez, C.A.; Li, Y. Numerical study of hybrid GFRP-steel reinforced concrete shear walls and SFRC walls. Eng. Struct. 2019, 180, 700-712. [CrossRef]

140. Ghazizadeh, S.; Cruz-Noguez, C.A. Damage-resistant reinforced concrete low-rise walls with hybrid GFRP-steel reinforcement and steel fibers. J. Compos. Constr. 2018, 22, 04018002. [CrossRef]

141. Athanasopoulou, A. Shear Strength and Drift Capacity of Reinforced Concrete and High-Performance Fiber Reinforced Concrete Low-Rise Walls Subjected to Displacement Reversals. Ph.D. Thesis, University of Michigan, Ann Arbor, MI, USA, 2010.

142. Tupper, B. Seismic Response of Reinforced Concrete Walls with Steel Boundary Elements. Ph.D. Thesis, McGill University Libraries, Montreal, QC, Canada, 1999.

143. Arafa, A.; Farghaly, A.; Benmokrane, B. Effect of web reinforcement on the seismic response of concrete squat walls reinforced with glass-FRP bars. Eng. Struct. 2018, 174, 712-723. [CrossRef]

144. Oesterle, R.G.; Fiorato, A.E.; Aristizabal-Ochoa, J.D.; Corley, W.G. Hysteretic Response of Reinforced Concrete Structural Walls. ACI Spec. Publ. SP63-11 1980, 63, 243-273.

145. Oesterle, R.; Fiorato, A.; Corley, W. Reinforcement details for earthquake-resistant structural walls. Concr. Int. 1980, 2, 55-66.

146. Shiu, K.; Daniel, J.; Aristizabal-Ochoa, J.; Fiorato, A.; Corley, W. Earthquake Resistant Structural Walls: Test of Walls With and Without Openings; NASA STI/Recon Technical Report N; NASA: Boston, MA, USA, 1981; Volume 82.

147. Jiang, H.J.; Lu, X.L. Calculating Method for the Hysteretic Characteristics of Shear Walls Dissipating Energy along the Vertical Direction. J. Tongji Univ. 1999, 6. [CrossRef]

148. Park, H.G.; Baek, J.W.; Lee, J.H.; Shin, H.M. Cyclic Loading Tests for Shear Strength of Low-Rise Reinforced Concrete Walls with Grade 550 MPa Bars. ACI Struct. J. 2015, 112, 299-310. [CrossRef]

149. Hube, M.; Santa María, H.; López, M. Experimental Campaign of thin Reinforced Concrete Shear Walls for Low-Rise Constructions. In Proceedings of the 16th World Conference on Earthquake Engineering, Santiago, Chile, 9-13 January 2017.

150. Alarcon, C.; Hube, M.; De la Llera, J. Effect of axial loads in the seismic behavior of reinforced concrete walls with unconfined wall boundaries. Eng. Struct. 2014, 73, 13-23. [CrossRef]

151. Kabeyasawa, T.; Matsumoto, K. Tests and analyses of ultra-high strength reinforced concrete shear walls. In Proceedings of the 10th World Conference on Earthquake Engineering, Madrid, Spain, 19-24 July 1992; pp. 3291-3296. 
152. Luna, B.N.; Rivera, J.P.; Whittaker, A.S. Seismic behavior of low-aspect-ratio reinforced concrete shear walls. ACI Struct. J. 2015, 112, 593. [CrossRef]

153. Altin, S.; Anil, Ö.; Kopraman, Y.; Kara, M.E. Hysteretic behavior of RC shear walls strengthened with CFRP strips. Compos. Part B Eng. 2013, 44, 321-329. [CrossRef]

154. Aaleti, S.; Brueggen, B.L.; Johnson, B.; French, C.E.; Sritharan, S. Cyclic response of reinforced concrete walls with different anchorage details: Experimental investigation. J. Struct. Eng. 2012, 139, 1181-1191. [CrossRef]

155. Segura, C.L., Jr.; Wallace, J.W. Seismic Performance Limitations and Detailing of Slender Reinforced Concrete Walls. ACI Struct. J. 2018, 115, 849-859. [CrossRef]

156. Devi, G.; Subramanian, K.; Santhakumar, A. Experimental investigations on reinforced concrete lateral load resisting systems under lateral loads. Exp. Tech. 2011, 35, 59-73. [CrossRef]

157. Dragan, D.; Plumier, A.; Degée, H. Experimental Study Regarding Shear Behavior of Concrete Walls Reinforced by Multiple Steel Profiles. In High Tech Concrete: Where Technology and Engineering Meet; Springer: Berlin/Heidelberg, Germany, 2018; pp. 1077-1084.

158. Ganesan, N.; Indira, P.; Seena, P. Reverse Cyclic Tests on High Performance Cement Concrete Shear Walls with Barbells. In Advances in Structural Engineering; Springer: Berlin/Heidelberg, Germany, 2015; pp. 2309-2321.

159. Liu, G.R.; Song, Y.P.; Qu, F.L. Post-fire cyclic behavior of reinforced concrete shear walls. J. Cent. South Univ. Technol. 2010, 17, 1103-1108. [CrossRef]

160. Lu, X.; Wang, D.; Zhao, B. Experimental Study on Seismic Performance of Precast Concrete Shear Wall with Joint Connecting Beam Under Cyclic Loadings. In Experimental Research in Earthquake Engineering; Springer: Berlin/Heidelberg, Germany, 2015; pp. 373-386.

161. Zhu, Z.; Guo, Z. Experimental study on emulative hybrid precast concrete shear walls. KSCE J. Civil Eng. 2017, 21, 329-338. [CrossRef]

162. Zhu, Z.; Guo, Z. Seismic Behavior of Precast Concrete Shear Walls with Different Confined Boundary Elements. KSCE J. Civil Eng. 2019, 23, 711-718. [CrossRef]

163. Barda, F.; Hanson, J.M.; Corley, W.G. Shear strength of low-rise walls with boundary elements. Spec. Publ. 1977, 53, 149-202.

164. Mansur, M.; Balendra, T.; H'ng, S. Tests on reinforced concrete low-rise shear walls under cyclic loading. In Concrete Shear in Earthquake; CRC Press: Boca Raton, FL, USA, 1992; pp. 14-16.

(C) 2019 by the authors. Licensee MDPI, Basel, Switzerland. This article is an open access article distributed under the terms and conditions of the Creative Commons Attribution (CC BY) license (http://creativecommons.org/licenses/by/4.0/). 